10-MWe Solar-Thermal

Central-Receiver Pilot Plant

SOLAR FACILITIES DESIGN INTEGRATION

\title{
SYSTEM INTEGRATION LABORATORY TEST PLAN (RADL ITEM 6-4)
}

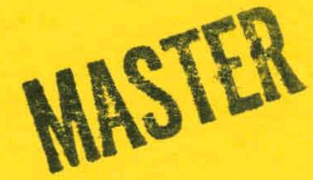

Revised October 1980 May 1980

WORK PERFORMED UNDER CONTRACT DE-AC03-79SF10499

MCDONNELL DOUGLAS ASTRONAUTICS COMPANY

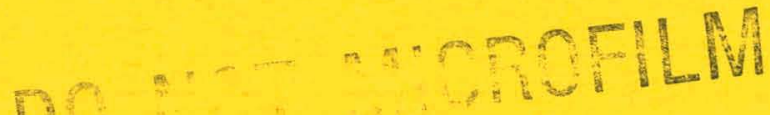

5301 BOLSA AVENUE

HUNTINGTON BEACH, CA 92647

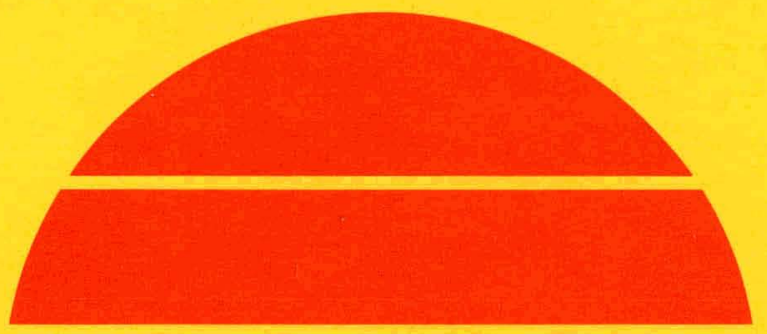

\section{U.S. Department of Energy}
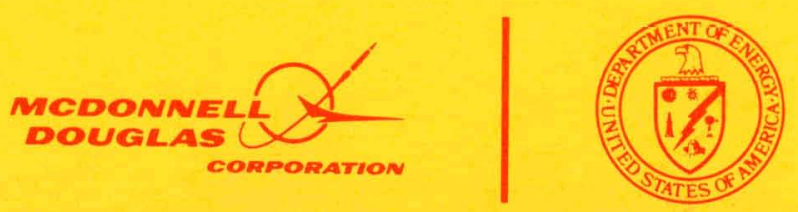


\section{DISCLAIMER}

This report was prepared as an account of work sponsored by an agency of the United States Government. Neither the United States Government nor any agency Thereof, nor any of their employees, makes any warranty, express or implied, or assumes any legal liability or responsibility for the accuracy, completeness, or usefulness of any information, apparatus, product, or process disclosed, or represents that its use would not infringe privately owned rights. Reference herein to any specific commercial product, process, or service by trade name, trademark, manufacturer, or otherwise does not necessarily constitute or imply its endorsement, recommendation, or favoring by the United States Government or any agency thereof. The views and opinions of authors expressed herein do not necessarily state or reflect those of the United States Government or any agency thereof. 


\section{DISCLAIMER}

Portions of this document may be illegible in electronic image products. Images are produced from the best available original document. 


\section{SYSTEM INTEGRATION LABORATORY TEST PLAN (RADL ITEM 6-4)}

\section{NOTICE}

\section{PORTIONS OF THIS REPORT ARE ILLEGIBLE, Revised October 1980}

has been reproduced from the best available

copy to permit the broadest possible avail.

ability.

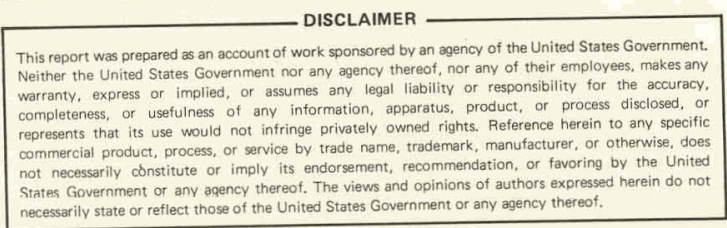

DISCLAIMER

This report was prepared as an account of work sponsored by the United States Government. Neither the United States nor the United States Department of Energy, nor any of their employees, makes any warranty, express or implied, or assumes any legal liability or responsibility for the accuracy, completeness, or usefulness of any information, apparatus, product, or process disclosed, or represents that its use would not infringe privately owned rights. Reference herein to any specific commercial product, process, or service by trade name, mark, manufacturer, or interite otherwise, does not necessarily constitute or imply its endorsement, recomment necessarily state or reflect those of the United States Government or any agency thereof.

\section{MCDONNELL DOUGLAS ASTRONAUTICS COMPANY} 5301 BOLSA AVENUE HUNTINGTON BEACH, CA 92647

PREPARED FOR THE U.S. DEPARTMENT OF ENERGY SOLAR ENERGY UNDER CONTRACT DE-AC-03-79SF10499

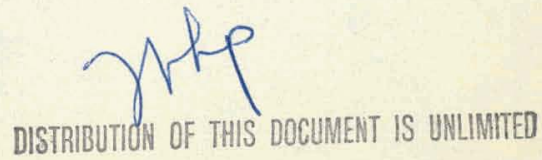




\section{PREFACE}

This document is provided by the McDonnel1 Douglas Astronautics Company (MDAC) in accordance with Department of Energy Contract No. DE-AC-03-79 SF 10499, Reports and Deliverables List, Item 6.4. The material contained describes the System Integration Laboratory Test Plan.

Questions concerning this document should be directed to J. C. Grosse (714) 896-4316. 


\section{CONTENTS}

Section 1

INTRODUCTION

1.1 Test Description Summary

1.1.1 MVCU Testing

1.1.2 Maxi SIL

1.1 .3 Mini SIL

1.1 .4 BCS SIL

Section 2 APPLICABLE DOCUMENTS

2.1 Reports and Deliverable List (RADL)

Documents

2.2 Interface Documents

2.3 Vendor Documents

2.4 Standard/Manuals

Section 3

SIL OPERATIONS AND REQUIREMENTS

3.1 Test Objectives

3.1.1 MVCU Objectives

3.1.2 SIL Objectives

3.1.3 Mini SIL Objectives 9

3.2 Test Facility

3.2.1 MVCU Testing

3.2.2 Maxi SIL

3.2.3 Mini SIL

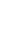

3.3 Schedule.

3.3.1 Maxi SIL Buildup and Test Scherilue

3.3.2 SIL Stage 0 Activities (SIL Preparation) 19

3.3.3 SIL Stage I Activities (Initial
DAS. Integration)

$\begin{array}{ll}3.3 .4 & \text { SIL Stage I I Activities (Initial } \\ & \text { OCS Integration) }\end{array}$

$\begin{array}{ll}3.3 .5 & \text { SIL Stage III Activities (SDPC } \\ \text { Integration) }\end{array}$

3.3.6 SIL Stage IV Activities

(Computer Update) 27

3.3.7 SIL Stage V Activities (SCU/RLU/
ILS Integration)

3.3.8 SIL Stage VI Activities (System
Evaluation) 


\subsubsection{SIL Stage VII Activities}

(Pack and Ship)

3.4 Test Configurations

3.4.1 MVCU Testing Configuration

3.4.2 Maximum SIL Test Configuration 31

3.4.3 Minimum SIL Test Configuration 58

3.5 Integration Testing 58

3.5.1 Maxi SIL 58

3.5.2 Mini SIL 60

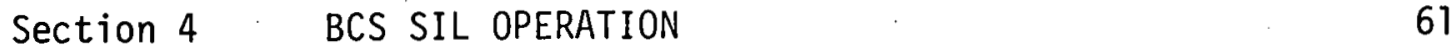

4.1 BCS Test Objective 61

4.2 BCS Test Facility 65

4.2.1 BCS Phase I Activities 65

4.2.2 BCS Phase II Activities 77

4.3 BCS Schedule 81 


\section{FIGURES}

1-1 Beckman Multivariable Controller Performance Test Configuration

1-2 SIL Plant Controls Test Configuration 2

1-3 Mini SIL Software Development Support Configuration 4

1-4 BCS Hardware Configuration 5

3-1 MVCU Facility Configuration 9

3-2 Maximum System Integration Laboratory 11

3-3 Equipment Configuration - MINI SIL 12

3-4 Systems Integration Laboratory Overall Activites Schedule

3-5 Maxi SIL Buildup and Test. Schedule 14

$\begin{array}{lll}3-6 & \text { SIL Software OCS Schedule } & 15\end{array}$

3-7 SIL DAS Software Integration Test Plan Schedule 16

3-8 DAS/OCS Interface Integration 17

3-9 Maxi SIL Test Configuration 32

3-jo OCS Computer Configuration 33

3-11 OCS Computer BTock Diagram (Initial Configuration) 34

3-12 OCS Computer Block Diagram (Operational Configuration) $\quad 35$

3-13 OCS Color CRT Terminat BTock Diagram 36

3-14 OCS/SDPC Interface 39

3-15 DAS Configuration 40

3-16 DAS Computer Block Diagram (Initial Configuration) 41

3-17. DAS Computer Block Diagram (Operational Configuration) 42

3-18 Data Acquisition Remote Multiplexer System 43 


\section{TABLES}

3-1 SIL Equipment Integration

3-2 Control and Instrumentation Equipment Modifications in SIL

3-4 System Functional Tests 


\section{GLOSSARY}

\begin{tabular}{|c|c|}
\hline AK & Annunciator Keyboard \\
\hline$B / W$ & Black and White \\
\hline BCS & Beam Characterization System \\
\hline C\&I & Controls and Instrumentation \\
\hline $\mathrm{CAB}$. & Cabinet \\
\hline CAM & Camera \\
\hline $\mathrm{CB}$ & Control Building \\
\hline CCM-PS & Communications Control Module Power Supply \\
\hline $\mathrm{CCP}$ & Communications and Control Processor \\
\hline $\mathrm{CCU}$ & Central Controlunit \\
\hline CFIC & Collector Field Interface Cabinet \\
\hline CTM & Communications Translator Module \\
\hline CLCU & Camera Lens ControlUnit \\
\hline $\mathrm{COHU}$ & Trademark of Video Systems Equipment Manufacturing Company \\
\hline CPU & Central Processing Unit \\
\hline CRT & Cathode Ray Tube \\
\hline CS & Collector Subsystem \\
\hline CSM & Configuration Storage Module \\
\hline DARMS & Data Acquisition and Remote Multiplexing System \\
\hline DAS & Data Acquisition System \\
\hline DBIOP & Dual Branch Input and Output Processor \\
\hline DEC 10 & Digital Equipment Corporation Computer Model Number \\
\hline DIR & Digital Image Radiometer \\
\hline DTMF & Dual Tone Modulating Frequency \\
\hline EAU & Extended Arithmetic Unit \\
\hline EER & Engineering Evaluation Room \\
\hline EES & Electronics Environmental Shelter \\
\hline EPGS & Electric Power Generating System \\
\hline EXT INT & External Interrupt \\
\hline GE & General Electric Company \\
\hline
\end{tabular}




\begin{tabular}{|c|c|}
\hline H COPIER & Hard Copier \\
\hline HAC & Heliostat Array Controller \\
\hline $\mathrm{HCP}$ & Host Communications Processor \\
\hline HTP & Historic Trend Processor \\
\hline HP & Hewlett Packard Company \\
\hline $\mathrm{HZ}$ & Hertz or Frequency \\
\hline $\mathrm{I} / \mathrm{F}$ & Interface \\
\hline $\mathrm{I} / 0$ & Input and Output \\
\hline IPAC & $\begin{array}{l}\text { Trademark of Digital Data Acquisition Equipment Manufacturing } \\
\text { Company }\end{array}$ \\
\hline IR & Infra Red \\
\hline ISC & Intelligent Systems Corporation \\
\hline $\mathrm{J}-\mathrm{B} 0 \mathrm{X}$ & Junction Box \\
\hline $\mathrm{KB}$ or $\mathrm{Kb}$ & Kilobit \\
\hline KBP & Keyboard Processor \\
\hline LPM & Lines Per Minute \\
\hline$M A / V$ & Milliamp to Voltage Converter \\
\hline $\operatorname{MAX}(I V)^{\circ}$ & Trademark of MODCOMP Operating System Software \\
\hline MAXNET (IV) & Trademark of MODCOMP Multicomputer Operating System Software \\
\hline MB or $\mathrm{Mb}$ & Mega Bit \\
\hline MCS & Master Control Subsystem \\
\hline MODACS & $\begin{array}{l}\text { Trademark of MODCOMP for Modular Data Acquisition and } \\
\text { Control Subsystem }\end{array}$ \\
\hline MODCOMP & Trademark of MODCOMP for Modular Computer Systems Incorporated \\
\hline MOS & Metal Oxide Semiconductor Memory \\
\hline MUX & Multiplexor \\
\hline MVCU & Multivariable Control Unit \\
\hline$N / A$ & Not Available \\
\hline OCS & Operational Control System \\
\hline OK & Operator Keyboard \\
\hline OLSF & On-Line Simulation Facility \\
\hline OPCU & $\begin{array}{l}\text { Operator Programmable Control Unit - Same as Operator } \\
\text { Programmable Interface Unit (OPIU) }\end{array}$ \\
\hline OPIU & Operator Programmable Interface Unit \\
\hline OSP & Operator Station Processor \\
\hline $\mathrm{P} / \mathrm{N}$ & Part Number \\
\hline PDP-10 & Digital Equipment Corporation Computer Model Number \\
\hline
\end{tabular}




$\begin{array}{ll}\text { PIP } & \text { Peripheral Interface Processor } \\ \text { RADL } & \text { Reports and Deliverables List } \\ \text { RAS } & \text { Remote Acquisition System } \\ \text { RGP } & \text { Report Generation Processor } \\ \text { RG (11) } & \text { Coaxial Wire Cable Type (11) } \\ \text { RI } & \text { Rockwell International } \\ \text { RLU } & \text { Red Line Unit } \\ \text { RMU } & \text { Remote Multiplexor Unit } \\ \text { RS } & \text { Receiver Subsystem } \\ \text { RS-232-C } & \text { Telecommunications Interface Standard Specification } \\ \text { RTT } & \text { Resistant Temperature Transmitter } \\ \text { S/C } & \text { Signal Conditioning } \\ \text { S/R } & \text { Stearns Roger Company } \\ \text { SCU } & \text { Signal Conditioning Unit } \\ \text { SDPC } & \text { Subsystem Distributed Process Control System } \\ \text { SETF } & \text { Solar Energy Test Facility } \\ \text { SHIMMS } & \text { Special Heliostat Instrumentation and Meteorlogical } \\ \text { SHM } & \text { Measurements System } \\ \text { SIL } & \text { Short Haul Modem } \\ \text { STA } & \text { System Integration Laboratory } \\ \text { SW } & \text { Station } \\ \text { T/G } & \text { Switch } \\ \text { TCD } & \text { Turbine Generator } \\ \text { TELCO } & \text { Test Control Drawing } \\ \text { TGT } & \text { Telephone Communications } \\ \text { TSS } & \text { Target } \\ \text { TV } & \text { Thermal Storage Subsystem } \\ \text { TWR } & \text { Television } \\ \text { V/MA } & \text { Tower } \\ \text { VAC } & \text { Voltage to Milliamp Converter } \\ \text { W/S } & \text { Alternating Current Voltage } \\ \text { WDS } & \text { Weather Station } \\ \text { WWV } & \text { Words } \\ & \text { Cail Letters of Time Standard } \\ & \end{array}$




\section{Section. 1}

INTRODUCTION

The purpose of this document is to provide a general demonstration test plan for the activities to be accomplished at the SFDI Systems Integration Laboratory (SIL) at Huntington Beach. The Master Control System (MCS), the Subsystem Distributed Process Control (SDPC), Representative Signal Conditioning Units (SCU), and Redline Units (RLU) from the Receiver Subsystem (RS) and the Thermal Storage Subsystem. (TSS) and other external interface operational functions will be integrated and functionally demonstrated. This document presents the overall plan for the SIL activities. Subsequent documents will present the detailed plans and procedures for integration and testing of the various subsystems.

\subsection{TEST DESCRIPTION SUMMARY}

The demonstration testing will be accomplished in four major phases.

A. Performance demonstration of Beckman. Multivariable Control Unit (MVCU)

B. Maximum System. Integration Laboratory (Maxi SIL)

C. Minimum System Integration Laboratory (Mini SIL)

D. BCS System Integration Laboratory

\subsubsection{MVCU Testing}

The Beckman MVCU will be tested for frequency response, static checks, configuration changes, switching transients, and input/output interfaces. Figure 1-1 indicates the configuration.

\subsubsection{Maxi SIL}

Maxi SIL testing will demonstrate the operational readiness of Pilot Plant controls and external interfaces that are available. Figure 1-2 is an overview block diagram of the test configuration. The subsystems will be installed and readiness tested. The various subsystems will then be interconnected and the interfaces tested to determine readiness to support integrated system 


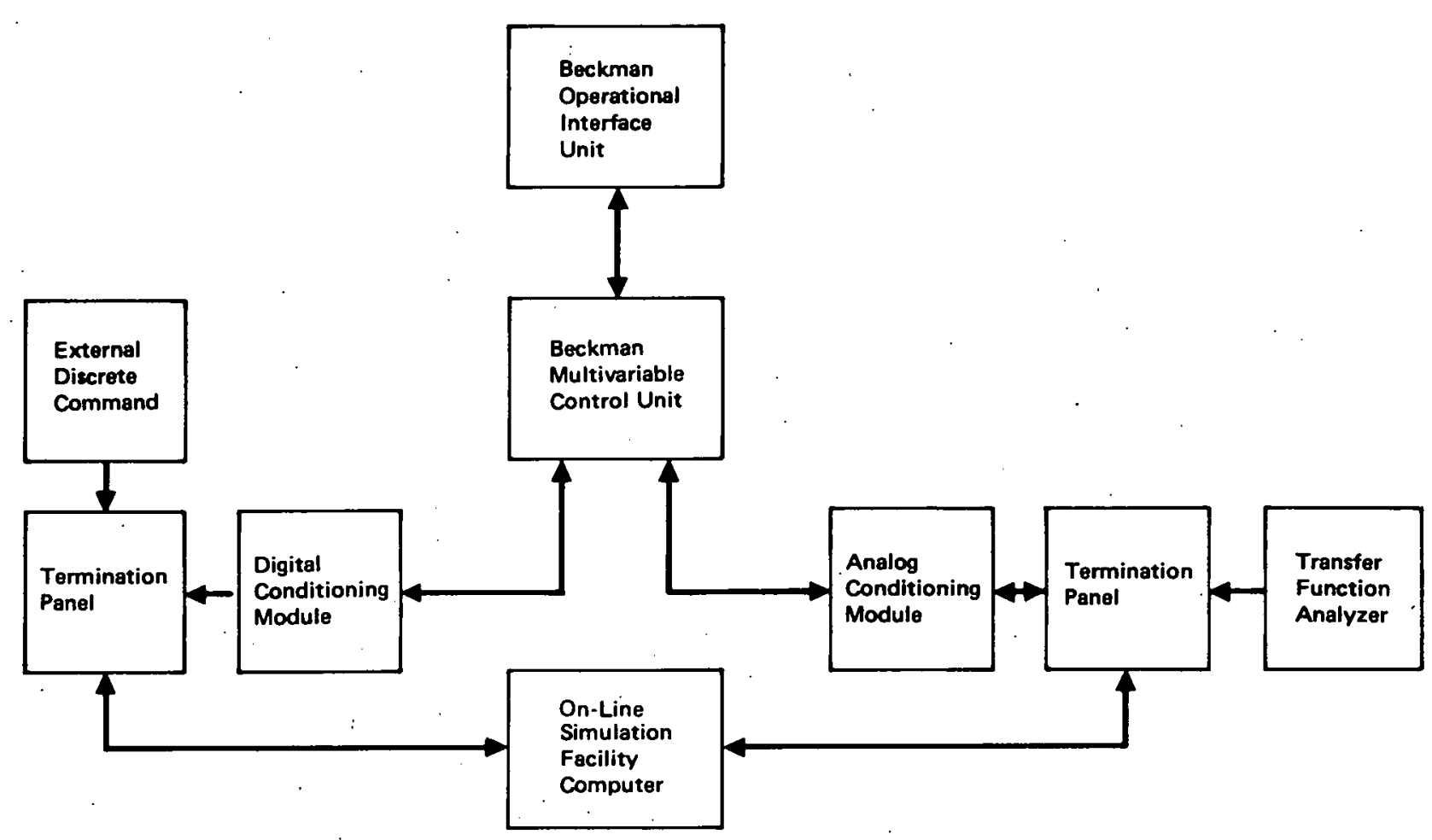

Figure 1-1. Beckman Multivariable Controller Performance Test Configuration

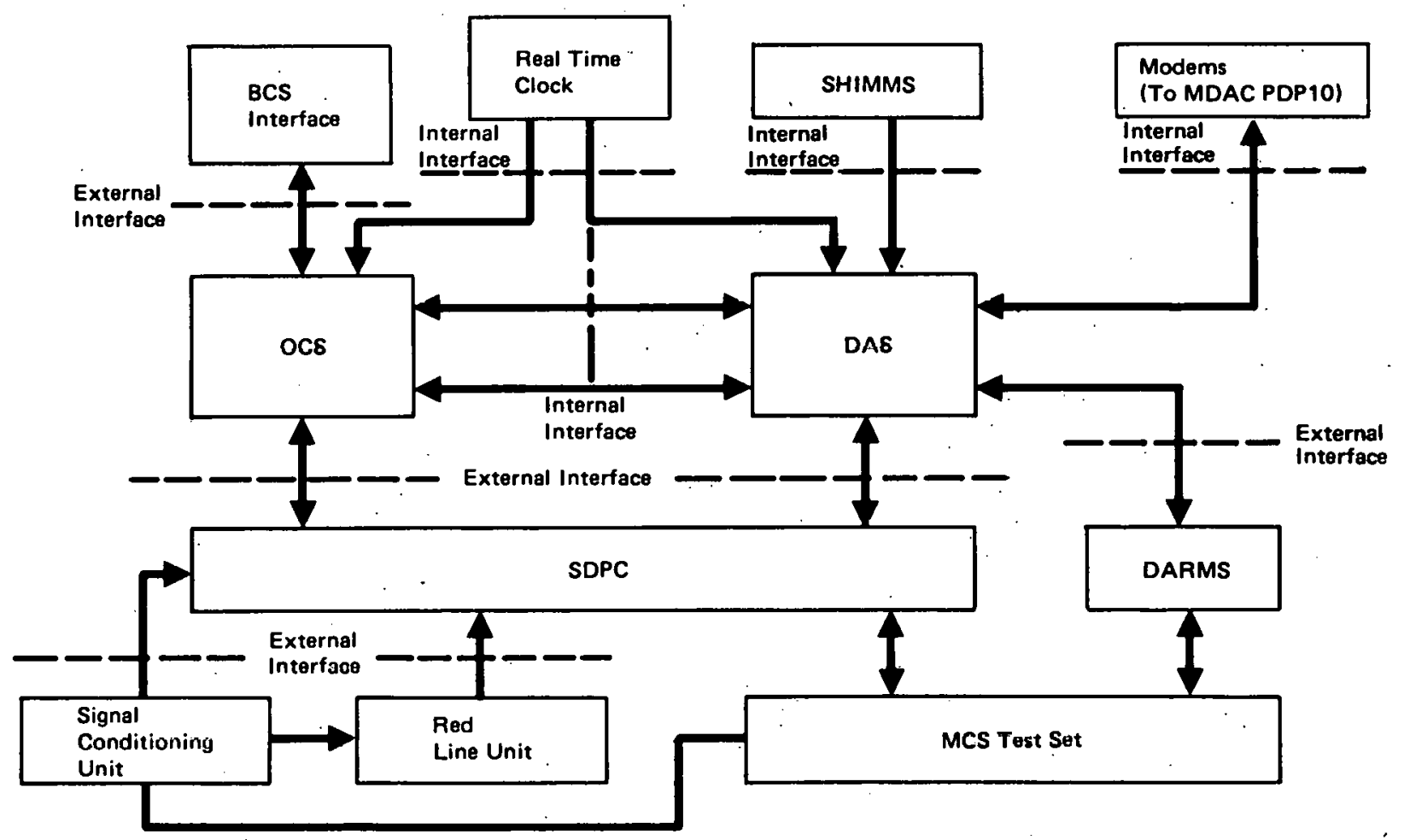

Figure 1-2. SIL Plant Controls Test Configuration 
demonstrations. Certain system-level testing will then be accomplished. Hardware, software, and firmware are involved in this activity.

\subsubsection{Mini SIL}

Mini SIL testing will be accomplished with a reduced (mini) set of hardware after delivery of equipment from maxi SIL to the site. The mini SIL hardware configuration, as illustrated in Figure 1-3, will. provide capability. for continued development and demonstration of Operational Control System (OCS) plant control application software. The oCS computer and its essential interfaces are provided in mini SIL so that the last increment of software can continue development and hardware/software integration can proceed while the system hardware is being installed, interconnected, and checked out at the site.

\section{1 .4 BCS SIL}

BCS SIL testing will demonstrate the operational readiness of the BCS equipment and software. Figure 1-4 provides an overview block diagram of the BCS hardware configuration. The components and software will be installed and interfaced to a surrogate OCS computer and target located in the Solar Energy Test Facility (SETF) at MDAC Huntington Beach and functionally tested. The system will then be installed in the MCS System Integration Laboratory with the final interfacing tests being run with the BCS software installed in the OCS computer. 


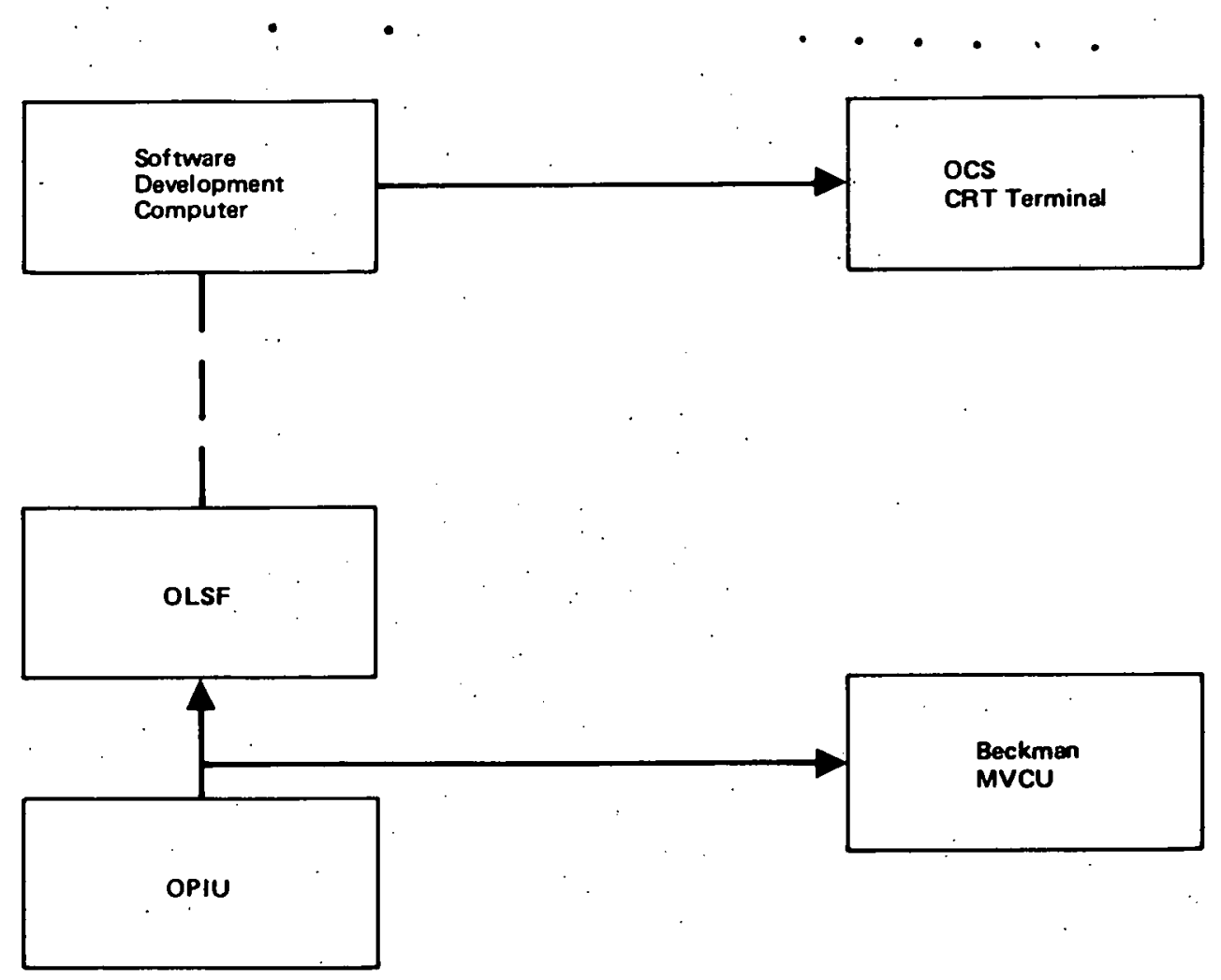

Figure 1-3. Mini SIL Software Development Support Configuration 
Section 2

APPLICABLE DOCUMENTS

\subsection{REPORTS AND DEL IVERABLE LIST (RADL) DOCUMENTS}

10 MW System Specification

(RADL Item 2-3) November 1979

MCS Hardware Design Specification

(RADL Item 6-1) Apri1 1980

MCS Software Design Specification

(RADL Item 6-1) Apri1 1980

\subsection{INTERFACE DOCUMENTS}

CS-MCS and CS-Plant Interface Requirements

(RADL Item 2-30-1) Preliminary June 1979

\subsection{VENDOR DOCUMENTS}

Reference Manual, MAX IV General Operating System, MODCOMP 210-610304-000; Revision E1.1, Jure 1979

Reference Manual, MAXNET IV, MODCOMP 210-610314-000, Revision 1 (E.1), July 1979

Classic Central Processor, MODCOMP 210-140000-000

TD-0780-01, Acceptance Test Procedure Data Acquisition System Operating Manual Cyber

Acceptance Test Procedure MV8000 Control System

Beckman

Operating Manual MV8000 Control System

Beckman

\section{2:4 STANDARDS/MANUALS}

Appendix A, Unit Development Folder, Appendix to MCS Software Project Plan, MDAC Drawing 1044799

Appendix B, Programming Standards, Appendix to MCS Software Project Plan, MDAC Drawing 1D44799

Appendix C, Flow Charting Standards and Guidelines; Appendix to MCS Software Project Plan, MDAC Drawing 1044799. 
Section 3

SIL OPERATIONS AND REQUIREMENTS

\subsection{TEST OBJECTIVES}

The objectives of the SIL activities is to demonstrate that Pilot Plant control equipment and software are compatible and ready for plant installation and integration. Systems and components to be tested in SIL are comprised of the OCS computer, OCS color CRTS, SDPC, ILS, SCU, RLU, BCS, DAS computer, DAS color CRT/printers, strip chart recorders, DARMS, SHIMMS, WWV receiver and time code generator, and special switch panels.

Systems and components which will not be tested in SIL because of unavailability during this time period are SHIMMS field instruments, turbine/ generator $(T / G)$ controls, trip logic box, $H A C$, circumsolar telescope, and the control building weather station equipment.

\subsubsection{MVCU Objectives}

The MVCU testing will"provide early assurance that algorithms as implemented within SDPC can meet functional and performance requirements. ParticuTar objectives are determination of accuracy of implemented algorithms at various frequencies; evaluation of nonlinear effects such as saturation and round off; effects of update rate; determination of switching transients due to gain, configuration, and mode changes.

\subsubsection{SIL Objectives}

The SIL operation will verify the mechanical and electrical integrity of the control system components. It will then verify proper component functioning and proper component interfacing with other components. Finally. system checks will demonstrate overall plant control system functions.

\subsubsection{Mechanical/Physical Integrity}

The first objective to be accomplished in the maxi SIL is to determine that the control equipment planned for the site equipment room and remote 
stations are compatible with their planned installations. Power input, cable lengths, terminations, air conditioning, equipment footprint, access, etc., will be examined.

\subsubsection{Electrical Integrity}

The second objective to be accomplished in maxi SIL is to determine power, grounding, and interface electrical compatibility of major control components.

\subsubsection{Component Functions}

The third objective is to demonstrate each major component functions in accordance with manufacturer's specification:

A.: Test with manufacturer's diagnostics.

B. Operation with manufacturer's procedures.

C. Operation with factory test procedures.

\subsubsection{Interface Functions}

The fourth objective is to evaluate subsystem internal and external interfaces (hardware and software). These interfaçes are:
A. SDPC/DAS computer
G. DAS displays/DAS computer
B. SDPC/OCS computer.
H. OCS displays/OCS computer
C. DARIIS/DAS computer
I. RLU/SDPC
D. SHIPIIIS/DAS computer
J. SCU/SDPC
E. Time-of-Day/DAS computer
K. SCU/DARMS
F. Time-of-Day/DAS computer
L. SCU/ILS

The fifth objective is to demonstrate the following plant control functions:
A. Operator interfaces
B. Input data processing
C. Output data processing Data displays/printing
D. Operating modes Data archiving
E. Tool software
F. Plant control configuring, displays, graphics 


\subsubsection{Mini SIL Objectives}

The mini SIL operation will provide the capability to continue development of the OCS control application software and modify or repair DAS software as required after the SIL equipment has been sent to the site.

\subsubsection{Application Software}

The essential interfaces between OCS and the plant control system will be proven in maxi SIL. The primary objective of the mini SIL is to complete the development of the OCS control application software. Figure l-3 indicates that sufficient hardware is present in mini SIL to continue development and demonstration of the OCS applications software.

\subsubsection{Modification of OCS/DAS Software}

Mini SIL will provide a local capability to modify the OCS/DAS software as required during initial checkout and startup operations, thus obviating extensive on-site TDY.

\subsection{TEST FACILITY}

\subsubsection{MVCU Testing}

MVCU tests will be accomplished in the On-Line Simulation Facility (OLSF) at MDAC-HB. The MVCU will interface with the OLSF analog computer as shown in Figure 3-1. Level changing isolation circuits are required to interface the two systems.

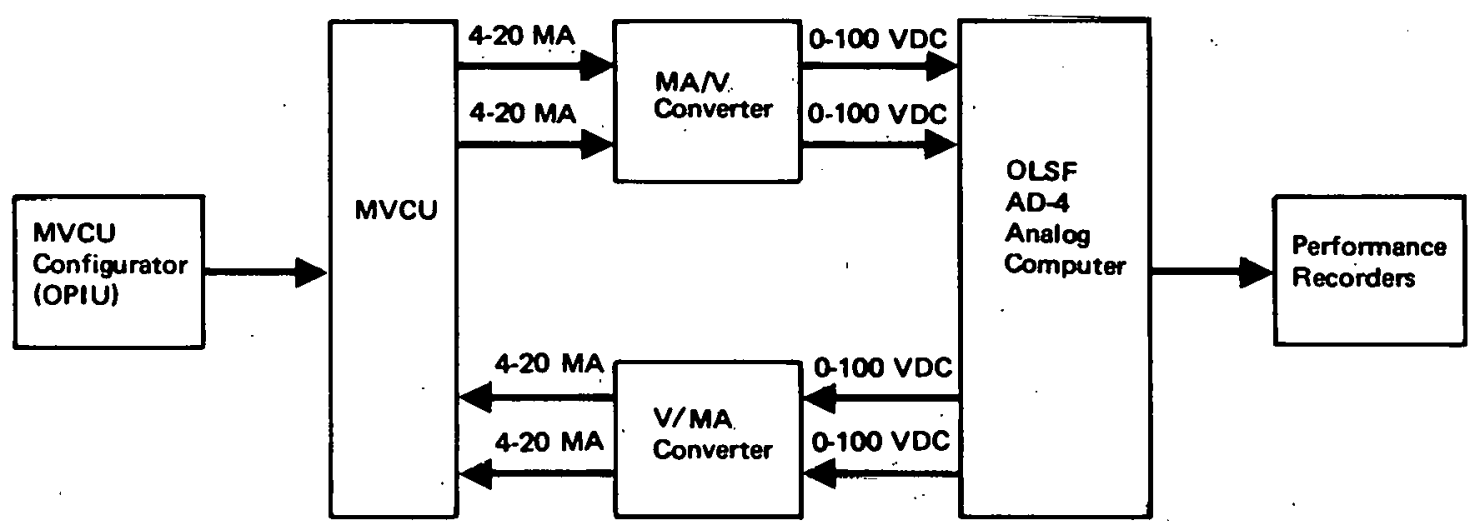

Figure 3-1. MVCU Facility Configuration 


\subsubsection{Maxi SIL}

Maxi SIL is located on the third floor of Building 22 at the MDAC-HB facility. The equipment configuration for the maxi SIL is shown in Figure 3-2. The SIL will provide a controlled-entry, air-conditioned area for:

A. Equipment receiving, assembly, and installation.

B. Fabrication of electrical/electronic assemblies, wiring, test cabling, test set, etc.

C. General-purpose test equipment.

D. Storage, office work area.

E. System testing.

\subsubsection{Mini SIL}

Mini SIL is comprised of facilities in two locations at MDAC-HB. A MODCOMP computer facility to support OCS software development and modifications to DAS computer software will be located on the first floor of Building 10 at the MDAC-HB facility. A Beckman Operator Programming Interface Unit (OPIU) and a Multivariable Control Unit (MVCU) to support evaluation and changes to control algorithms will be located in the OLSF in Building 22 at MDAC-HB. The equipment configuration for the mini SIL located in Building 10 is shown in Figure 3-3. The SIL will provide a controlled-entry, airconditioned area for equipment installation and system testing.

The equipment configuration for the mini SIL located in Building 22 is shown in Figure 3-1.

\subsection{SCHEDULE}

The beginning of maxi SIL is expected in October 1980. The end is scheduled on March 16, 1981. Figure 3-4 indicates the planned schedule for the maxi SIL, BCS SIL, and mini SIL phases of the SIL program.

\subsubsection{Maxi SIL Buildup and Test Schedule}

The actual plan for buildup and test of the maxi SIL is shown in Figures 3-5 through 3-8. The key milestones are: installation of the initial DAS computer the first week of October followed closely by installation of the initial OCS computer; installation of the SDPC equipment the first 


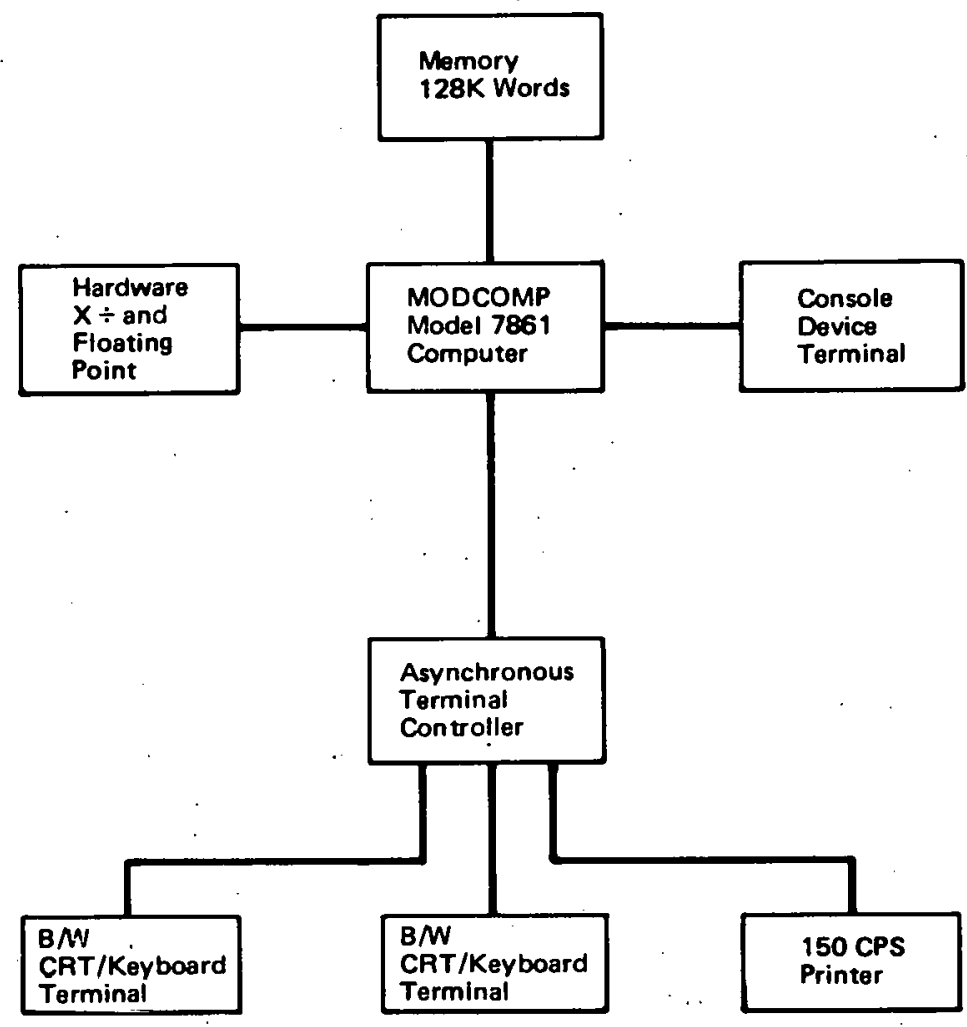

Figure 3-3. Equipment Configuration - MINI SIL

week in December; update of the DAS and OCS computers to the operational configurations in mid-December; completion of all equipment installation and checkout by the first week in February; completion of system evaluation by the first week in March; and delivery of the equipment to the plant site by the first of April 1981.

The maxi SIL activities are segmented into eight stages as shown in Figure 3-5. The stages are based on expected arrival dates of the primary hardware components. In some instances, the stage activities overlap in time. The relationship of the hardware systems and components to the SIL stages is shown in Table 3-1. 


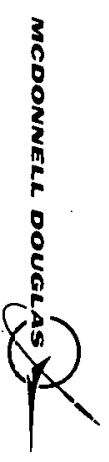

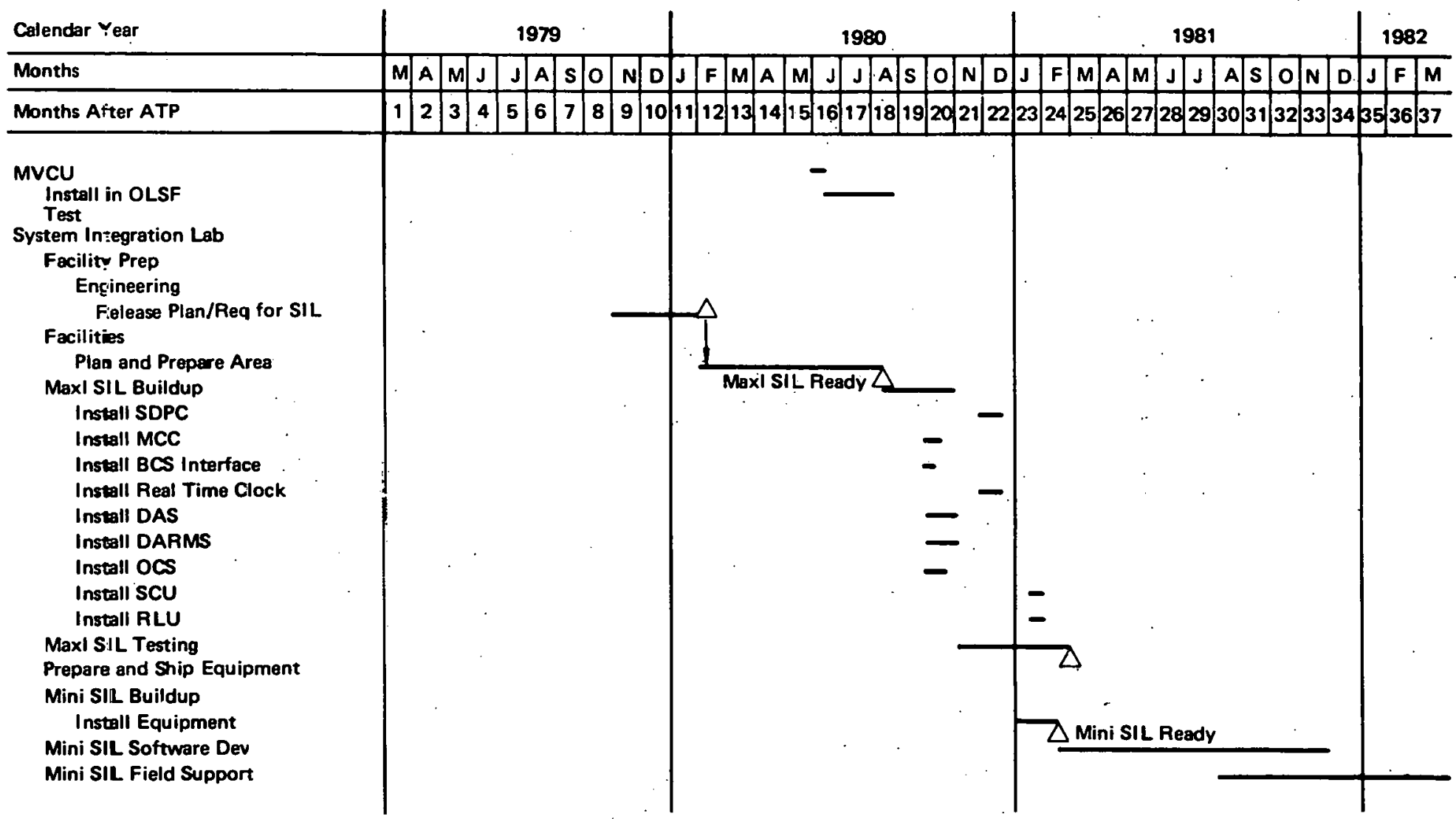

Figure 3-4. Systems Integration Laboratory Overall Activities Schedule 


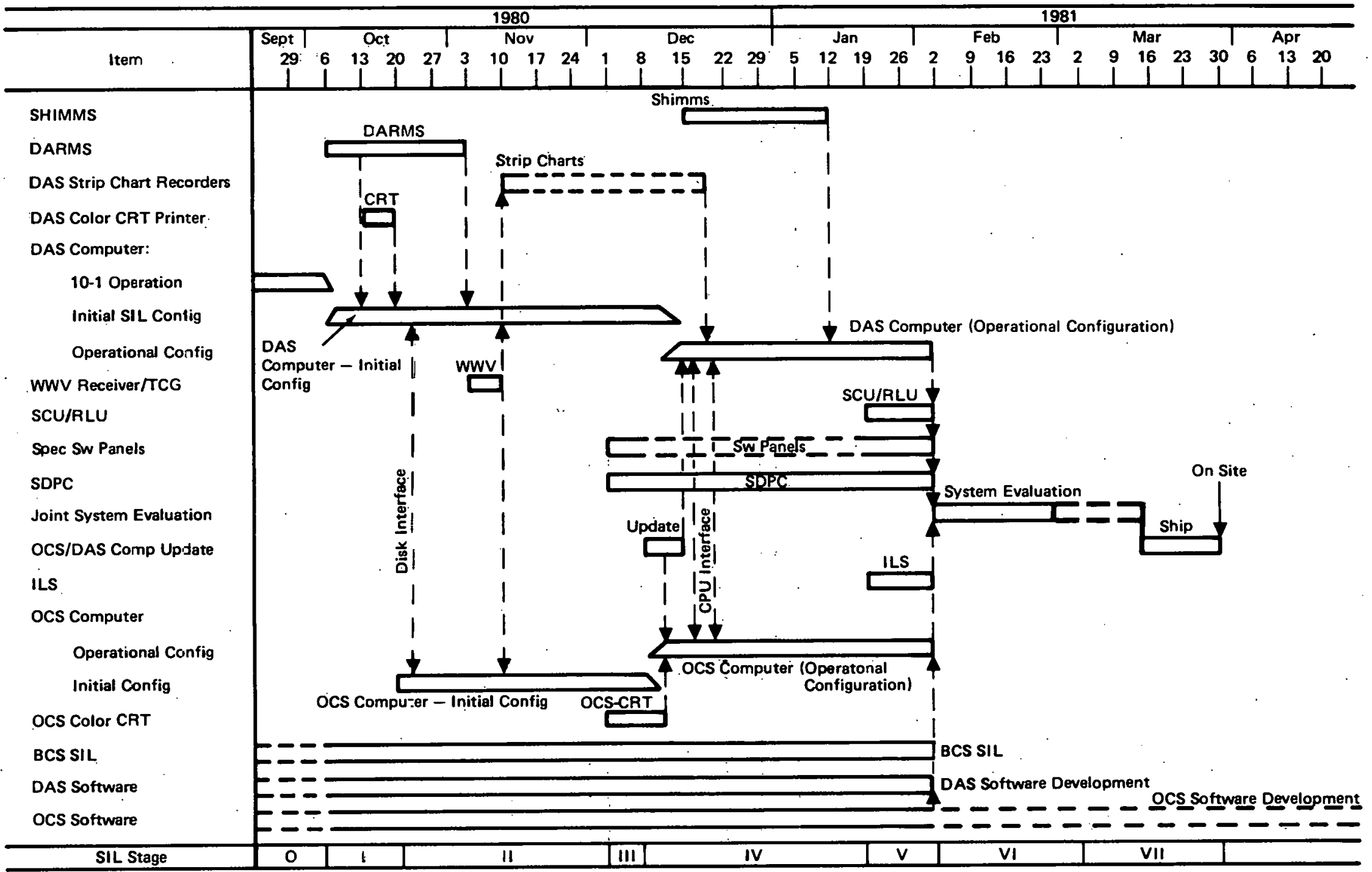

Figure 3-5. Maxi SI L Buildup and Test Schedule 


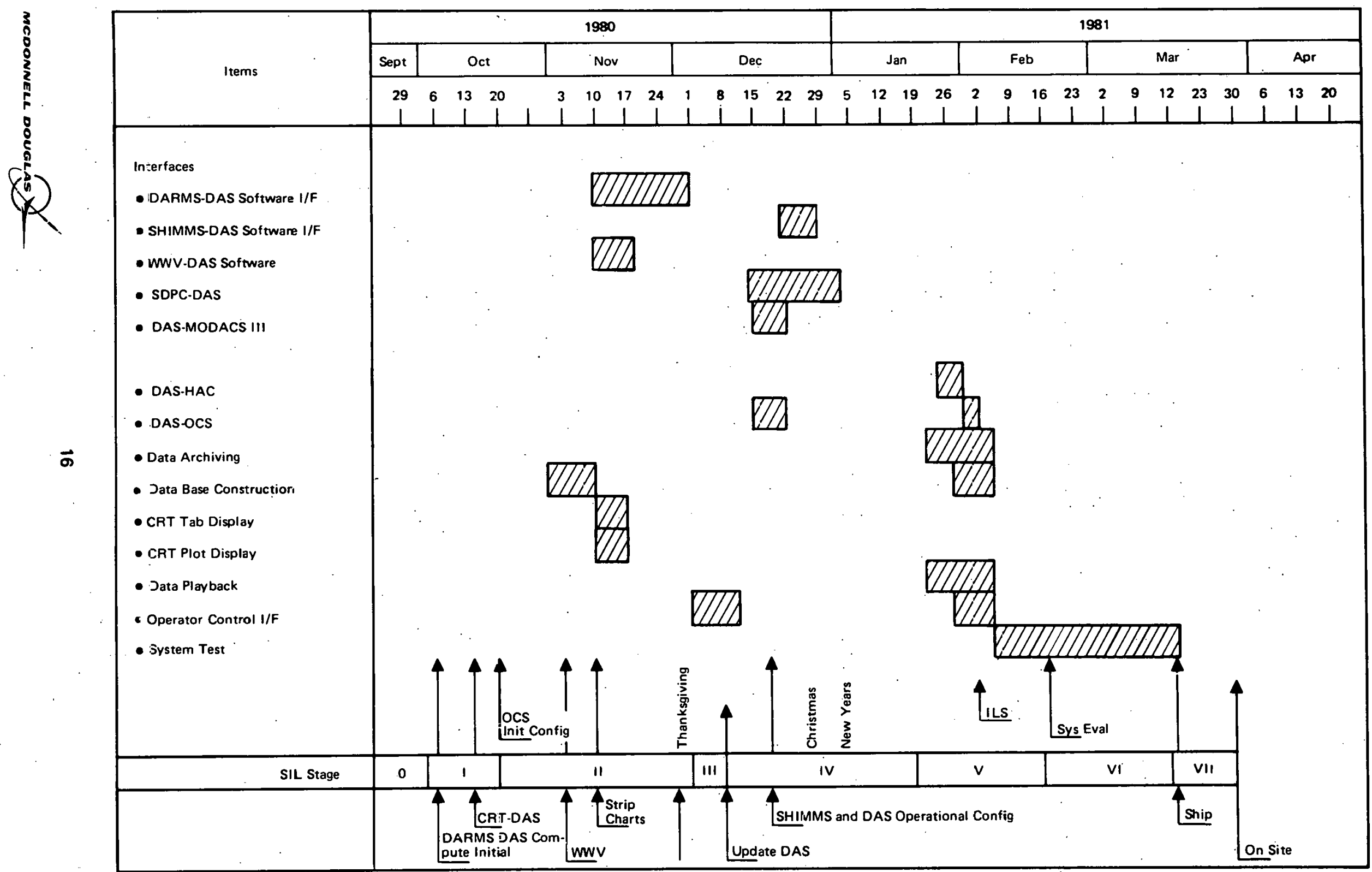

Figure 3-7. SIL DAS Software Integration Test Plan Schedule 
Table 3-1. SIL Equipment Integration (Page 1 of 2)

\begin{tabular}{|c|c|c|c|c|c|c|c|c|c|}
\hline \multirow[b]{2}{*}{ Seq } & \multirow[b]{2}{*}{ I tem } & \multirow[b]{2}{*}{ Supplier } & \multirow{2}{*}{$\begin{array}{l}\text { Delivery. } \\
\text { date }\end{array}$} & \multicolumn{5}{|c|}{ Stage } & \multirow[b]{2}{*}{ Remarks* } \\
\hline & & & & $\mathrm{I}$ & II & III & IV & V & \\
\hline 1 & Basic OCS Computer & Modcomp & $10-20-80$ & & $\star$ & & & & 2,3 \\
\hline $1 A$ & $\begin{array}{l}\text { OCS Computer add- } \\
\text { on }\end{array}$ & Modcomp & $12-8-80$ & & & & * & & 2 \\
\hline IB & OCS Color CRTs & Beckman & $12-1-80$ & & & * & & & \\
\hline 2 & $\begin{array}{l}\text { SDPC Consoles/ } \\
\text { Remotes }\end{array}$ & Beckman & $12-1-80$ & & & * & & & \\
\hline 3 & ILS & Beckman & $1-15-81$ & & & & & * & \\
\hline 4 & SCU & Rocketdyne & $1-15-80$ & & & & & * & \\
\hline 5 & RLU & Rocketdyne & $1-15-80$ & & & & & * & \\
\hline 6 & BCS & MDAC & $2-2-80$ & & & & & * & 8 \\
\hline 7 & Basic DAS Computer & Modcomp & in-house & $\star$ & & & & & 1,2 \\
\hline $7 \mathrm{~A}$ & $\begin{array}{l}\text { DAS Computer add- } \\
\text { on }\end{array}$ & Modcomp & $12-8-80$ & & & & * & & 2 \\
\hline $7 \mathrm{~B}$ & $\begin{array}{l}\text { DAS Color CRTs/ } \\
\text { Printers }\end{array}$ & ISC & $10-13-80$ & $\star$ & & & & & \\
\hline $7 C$ & $\begin{array}{l}\text { Strip Chart } \\
\text { Recorders }\end{array}$ & Gould & $11-10-80$ & & * & & & & .9 \\
\hline 8 & DARMS & Cyber & $10-6-80$ & * & & & & & 4,5 \\
\hline 9 & SHIMMS & Cyber & $12-15-80$ & & & & * & & \\
\hline $9 A$ & $\begin{array}{l}\text { SHIMMS field } \\
\text { instruments }\end{array}$ & & $\mathrm{N} / \mathrm{A}$ & & & & & & 6 \\
\hline 10 & WWV Receiver/TCG & System Donner & $11-3-80$ & & $\star$ & & & & 7 \\
\hline 11 & $T / G$ Control & $G E$ & $N / A$ & & & & & & 6 \\
\hline 12 & Trip Logic & $S / R$ & $N / A$ & & & & & & 6 \\
\hline 13 & HAC & Martin & $N / A$ & & & & & & 6 \\
\hline 14 & $\begin{array}{l}\text { Special Switch } \\
\text { Panels }\end{array}$ & Beckman & $12-1-80$ & & & & & & 10 \\
\hline 15 & $\begin{array}{l}\text { Circumsolar } \\
\text { Telescope }\end{array}$ & & N/A & & & & & & 6 \\
\hline 16 & IR Camera & & $N / A$ & & & & & & 6 \\
\hline 17 & $\begin{array}{l}\text { Cont Build } \\
\text { Weather Sta }\end{array}$ & & $N / A$ & & & & & & 6 \\
\hline 18 & Plant Devices & & $\mathrm{N} / \mathrm{A}$ & & & & & & 6 \\
\hline
\end{tabular}


Table 3-7. SIL Equipment Integration (Page 2 of 2)

*Remarks :

1. Basic DAS computer to be moved from 10-1 to 22-3.

2. Relocate power panels to afford bottom cable access to cabinets.

3. Basic OCS computer will be initially installed without communications processor for asynchronous RS232 communication controller.

4. Add termination resistors and jumpers.

5. Add circuit for interrupt line to DAS computer.

6. $N / A$ indicates equipment will not be available for integration into SIL activities .

7. Install in DARMS CCU cabinet for SIL.

8. BCS development by MDAC will proceed in parallel with and independent of SIL using surrogate OCS computer. Software to be tested with MAXNET operating system in SIL OCS computer during Stage $V$.

9. Strip chart recorders will be integrated with MODACS-III in Stage IV.

10. Switch panels will be integrated with ILS and RLU in Stage V.

A description of the activities to be performed for each stage is given in the following paragraphs.

\subsubsection{SIL Stage 0 Activities (SIL Preparation)}

The following activities will be performed during Stage 0 .

A. Prepare SIL facility.

1. Define and install electrical power for SIL equipment.

2. Define and obtain furniture.

3. Define and install telephones.

4. Define, install, and checkout four wire lines from SIL area to software development terminal area.

5. Define and install door locks.

B. Prepare SIL equipment layouts.

1. Physical location.

2. Electrical power cable routing.

3. Signal cable routing. 
c. Define SIL unique cable requirements.

1. Power

2. Signal

D. Fabricate Stage I cables.

1. Power

2. Signal

E. Prepare SIL area floor.

1. Clean

2. Mark equipment locations

3. Cut cable access holes in floor tiles for Stage I equipment.

F. Define SIL support equipment requirements for:

1. J-Box A substitute

2. Temporary mounting racks for SHIMMS remote acquisition (RAS)

units .

3. Plant interface checkout devices for: SDPC; DARMS; ILS; SHIMMS; SCU; RLU; Modems for MDAC data link and plant trip logic

4. Interface checkout devices for plant control and instrumentation (C\&I) equipment not available for SIL integration including: T/G Control Unit. and weather stations

G. Fabricate SIL support equipment for Stage I.

H. Prepare for move of initial DAS computer to SIL area.

I. Define and arrange for consumable supplies.

J. Set up Stage I equipment log books.

K. Propare Test Control Drawing for Stage I.

L. Define and fabricate power panel extension mounts for MODCOMP r.omput.er r.ahinet.s.

\subsubsection{SIL Stage I Activities (Initial DAS Integration)}

The following activities will be performed during Stage I:

A. Move initial DAS computer from temporary area to SIL area and run standard MOUCOMP computer functional tests.

B. Remove bottom screen from MODCOMP computer cabinets and relocate power panels using new extension mounts.

c. Receive, install, and checkout Stage I equipment identified in Table 3-1. 
D. Perform Stage I equipment additions/modifications as shown in Table 3-2.

E. Connect and checkout Stage I interfaces in accordance with Table 3-3.

F. Connect DAS computer to software development terminals via four wire lines and checkout.

G. Fabricate Stage II cables.

H. Fabricate Stage II SIL support equipment.

I. Set up Stage II equipment test log books.

J. Maintain equipment test log books.

\subsubsection{SIL Stage II Activities (Initial OCS Integration)}

The following activities will be performed during Stage II:

A. Receive, insta11, and checkout Stage II equipment as shown in Table 3-1.

B. Perform Stage II equipment additions/modifications as shown in Table 3-2.

c. Connect and checkout Stage II interfaces in accordance with Table 3-3.

D. Fabricate Stage III cabies.

Table 3-2. Control and Instrumentation Equipment Modifications in SIL

\begin{tabular}{|c|c|c|c|c|c|c|}
\hline \multirow[b]{2}{*}{ Scq } & \multirow[b]{2}{*}{ Description } & \multicolumn{5}{|c|}{ SIL Stage } \\
\hline & & $\mathrm{I}$ & II & I I I & IV & V \\
\hline 1 & $\begin{array}{l}\text { Modify Modcomp computer cabinets for } \\
\text { bottom cable access }\end{array}$ & * & * & & & \\
\hline 2 & Add terminations to DARMS RMUs & * & $\cdot$ & & & \\
\hline 3 & $\begin{array}{l}\text { Add } 5 \text {-vult output circult to DARMS } \\
\text { CCU for DAS computer external } \\
\text { interrupt }\end{array}$ & * & & & & \\
\hline 4 & $\begin{array}{l}\text { Add component ID tags to equipment } \\
\text { cabinets and cables }\end{array}$ & & & & & * \\
\hline
\end{tabular}


Table 3-3. SIL Interface Checkout Plan (Page 1 of 5)

\begin{tabular}{|c|c|c|c|c|c|c|c|c|c|c|}
\hline \multirow[b]{2}{*}{ Seq } & \multicolumn{3}{|r|}{ Interface } & \multirow[b]{2}{*}{ Qty } & \multicolumn{5}{|c|}{ SIL Stage } & \multirow[b]{2}{*}{ Remarks* } \\
\hline & From & To & Description & & I & II & I I I & IV & V & \\
\hline 1 & OCS & OCS & Control Room Color CRTs & 2 & & & & 4.1 & & \\
\hline$i$ & OCS & OCS & Control Room Matrix Printer & 1 & & 2.1 & & & & \\
\hline$\Xi$ & OCS & OCS & $\begin{array}{l}\text { Engineering Evaluation Room } \mathrm{B} / \mathrm{W} \\
\text { CRTs }\end{array}$ & 2 & & 2.2 & & & & \\
\hline 4 & OCS & OCS & Console Device & 1 & & 2.3 & & & & \\
\hline 5 & OCS & OCS & Magnetic Tape Drive & 1. & & 2.4 & & & & \\
\hline 6 & OCS & OCS & 10 MB Disk & 1 & & 2.5 & & & & \\
\hline 7 & OCS & OCS & 67 MB Disk. & 2 & & 2.6 & & & & 1 \\
\hline 8 & OCS & SDPC & Data Link & 6 & & & & 4.2 & & \\
\hline 9 & OCS & $\mathrm{BCS}$ & Digitizer data link & $\because 1$ & & . & & & & 5 \\
\hline 10 & OCS & $\mathrm{BCS}$ & $\begin{array}{l}\text { Dual Tone Modulating Frequency } \\
\text { (DTMF) data link }\end{array}$ & 1 & & & & & & 5 \\
\hline 11 & OCS & $\mathrm{BCS}$ & Link to modem for Modacs III & 1 & & & & & & 5 \\
\hline 12 & OCS & $\mathrm{BCS}$ & BCS CRT/Copier & 1 & & & & & & 5 \\
\hline 13 & OCS & DAS & OCS CPU-DAS CPU data link & 1 & & & & 4.3 & & \\
\hline 14 & ocs & WWV & Time of year data to OCS & 1 & & & & $4: 4$ & & \\
\hline 15 & OCS & HAC & CPU-CPU data link & 2 & & & & 4.5 & & 6 \\
\hline 16 & SDPC & SDPC & Console CRTs & 3 & & . & 3.1 & & & \\
\hline 17 & SDPC & SDPC & Console Loggers & 3 & & & 3.2 & . & & . \\
\hline 18 & SDPC & SDPC & Console Strip Chart Recorders & 3 & & & 3.3 & & & \\
\hline 19 & SDPC & SDCP. & MVCUs (at 4 Remote Stations) & 21 & & & 3.4 & & & \\
\hline 20 & SDPC & SDPC & $\begin{array}{l}\text { Mu'tiplexer Units (at } 4 \text { Remote } \\
\text { Stations) }\end{array}$ & 8 & & & 3.5 & & & \\
\hline
\end{tabular}


Table 3-3. SIL Interface Checkout Plan (Page 2 of 5)

\begin{tabular}{|c|c|c|c|c|c|c|c|c|c|c|}
\hline \multirow[b]{2}{*}{ Seq } & \multicolumn{3}{|r|}{ Interface } & \multirow[b]{2}{*}{ Qty } & \multicolumn{5}{|c|}{ SIL Stage } & \multirow[b]{2}{*}{ Remarks* } \\
\hline & From & To & Description & & $\mathrm{I}$ & II & III & IV & V & \\
\hline 21 & SDPC & ILS & Data link to MODICON 584 Units & 3 & & & & & 5.1 & \\
\hline 22 & SDPC & ILS & Data link to IPAC Units & 3 & & & & & 5.2 & \\
\hline 23 & SDPC & SCU & $\begin{array}{l}\text { Measurement data to MVCU (at } \\
\text { Remote Station) }\end{array}$ & 3 & & & & & 5.3 & \\
\hline 24. & SDPC & SCU & $\begin{array}{l}\text { Measurement data to MUX (at } \\
\text { Remote Station) }\end{array}$ & 3 & & & & & 5.4 & \\
\hline 25 & SDPC & DAS & Data Iink & 6 & & & & 4.6 & & \\
\hline 26 & SDPC & Trip Logic & Trip data to Station 4 MUX & 1 & & & & 4.7 & & 7 \\
\hline 27 & SDPC & Plant & MVCU commands and data & 4 & & & & 4.8 & & 8 \\
\hline 23 & SDPC & Plant & NUX data & 4 & & & & 4.9 & & 8 \\
\hline 29 & ILS & ILS & ILS remote units & 5 & & & & & 5.5 & \\
\hline 30 & ILS & $\operatorname{SCU}$ & Measurement data to ILS & 3 & & & & & 5.6 & \\
\hline 31 & ILS & RLU & Manual reset commands & 2 & & & & & 5.7 & \\
\hline 32 & ILS & Trip Logic & Steam Dump System reset command & 1 & & & & & 5.8 & 7 \\
\hline 33 & ILS & Sw Panels & $\begin{array}{l}\text { Manual commands to ILS via } \\
\text { Modicon } 584\end{array}$ & 4 & & & & & 5.9 & \\
\hline 34 & ILS & Sw Panels & Manual commands to SDPC via IPAC & 4 & & & & & 5.10 & \\
\hline 35 & $\operatorname{SCU}$ & RLU & Measurement data to RLU & 2 & & & & & 5.11 & \\
\hline 36 & $\mathrm{SCU}$ & DARMS & Measurement data to DARMS. RMU & 3 & & & & & 5.12 & \\
\hline $3 i$ & RLU & DARMS & High-speed scan interrupt & 2 & & & & & 5.13 & \\
\hline 38 & RLU & Trip Logic & Trip indications & 2 & & & & & 5.14 & 7 \\
\hline 39 & RLU & HAC & Trip indication & 1 & & & & & 5.15 & 6 \\
\hline 40 & RLU. & Sw Panels & Trip commands & 4 & & & & & 5.16 & \\
\hline
\end{tabular}


Table 3-3. SIL Interface Checkout Plan (Page 3 of 5 )

\begin{tabular}{|c|c|c|c|c|c|c|c|c|c|c|}
\hline \multirow[b]{2}{*}{ Seq } & \multirow[b]{2}{*}{ From } & \multicolumn{2}{|r|}{ Interface } & \multirow[b]{2}{*}{ Qty } & \multicolumn{5}{|c|}{ SIL Stage } & \multirow[b]{2}{*}{ Remarks* } \\
\hline & & To & Description & & I & II & III & IV & V & \\
\hline 41 & $\mathrm{BCS}$ & $\mathrm{BCS}$ & Control room video monitor & 1 & & & & & & 5 \\
\hline 42 & $\mathrm{BCS}$ & BCS & TV Cameras & 4 & & & & & & 5 \\
\hline 43 & $\mathrm{BCS}$ & BCS & -V Control receivers & 4 & & & & & & 5 \\
\hline 44 & $\mathrm{BCS}$ & $\mathrm{BCS}$ & Target Pyrheliometer data & 16 & & & & & & 5 \\
\hline 45 & $\mathrm{BCS}$ & $\mathrm{BCS}$ & Target Shutter controls & .16 & & & & & & 5 \\
\hline 46 & BCS & $\mathrm{BCS}$ & Modem to Station MODACS-III & 1 & & & & & & 5 \\
\hline 47 & DAS & DAS & Strip chart data to MODACS III & 1 & & & & 4.10 & & \\
\hline 48 & DAS & DAS & Analog signals to strip charts & 24 & & & & 4.11 & & \\
\hline 49 & DAS & DAS & Data link to DEC 10 at MDAC (A3) & 3 & & & & 4.12 & & 9 \\
\hline 50 & DAS & DAS & Display data to color CRTs & 2 & 1.1 & & & & & \\
\hline 51 & DAS & DAS & $\begin{array}{l}\text { Output data to CRT printer/ } \\
\text { plotter }\end{array}$ & 2 & 1.2 & & & & & \\
\hline 52 & DAS & DAS & E/W CRT Terminals & 2 & 1.3 & & & & & \\
\hline 53 & DAS & DAS & Matrix printer & 1 & 1.4 & & & & & \\
\hline 54 & DAS & DAS & $6: 00 \mathrm{Lpm}$ printer & 1 & 1.5 & & & & & \\
\hline 55 & DAS & DAS & Console device & 1 & 1.6 & & & & & \\
\hline $5 \bar{b}$ & DAS & DAS & Magnetic tape drive & 2 & 1.7 & & & & & \\
\hline 57 & DAS & DAS & E7 MB disk & 2 & 1.8 & & & & & 1 \\
\hline 58 & DAS & DAS & CRT programming terminals & 2 & 1.9 & & & & & 2 \\
\hline 59 & DAS & DARMS & Cata link & 1 & 1.10 & & & & & \\
\hline 60 & DAS & DARMS & Command link & 1 & 1.11 & & & & & \\
\hline 61 & DAS & DARMS & High-speed scan interrupt & 1 & 1.12 & & & & & \\
\hline
\end{tabular}


Table 3-3. SIL Interface Checkout Plan (Page 4 of 5)

\begin{tabular}{|c|c|c|c|c|c|c|c|c|c|c|}
\hline \multirow[b]{2}{*}{ Seq } & \multicolumn{3}{|r|}{ Interface } & \multirow[b]{2}{*}{ Oty } & \multicolumn{5}{|c|}{ SIL Stage } & \multirow[b]{2}{*}{ Remarks } \\
\hline & From & To & Description & & I & II & III & IV & v & \\
\hline 62 & DAS & SHIMMS & Jata link & 1 & & & & 4.13 & & \\
\hline 63 & DAS & SHIMMS & Command link & 1 & & & & 4.14 & & \\
\hline 64 & DAS & WWV & Time of year data to DAS & 1 & & 2.7 & & & & \\
\hline 65 & DAS & WWV & Serial time code to $S / C$ recorders & 1 & & 2.8 & & & & \\
\hline 66 & DAS & HAC & CPU-CPU data link & 1 & & & & 4.15 & & 6 \\
\hline 67 & DARMS & DARMS & CCU CRT Terminal & 1 & 1.13 & & & & & \\
\hline$€ 8$ & DARMS & DARMS & Remote Station RMUs & 8 & 1.14 & & & & & \\
\hline 69 & DARMS & $T / G$ Control & High-speed scan Interrupt & 1 & 1.15 & & & & & 10 \\
\hline 70 & SHIMMS & SHIMMS & CRT Terminal & 1 & & & & 4.16 & & \\
\hline 71 & SHIMMS & SHIMMS & $\begin{array}{l}\text { Sta-1 Remote Acquisition System } \\
\text { (Tower weather station) }\end{array}$ & 1. & & & & 4.17 & & \\
\hline 72 & SHIMMS & SHIMMS & RAS & 1 & & & & 4.18 & & 3 \\
\hline 73 & SHIMMS & SHIMMS & $\begin{array}{l}\text { Collector field Remote Acquisi- } \\
\text { tion Systems }\end{array}$ & 4 & & & & 4.19 & & 4 \\
\hline 74 & WWV & WWV & Antenna & 1 & & 2.9 & & & & \\
\hline 75 & $T / G$ Control & $T / G$ Control & Console operator panel & 1 & & & & & & 11 \\
\hline 76 & $T / G$ Control & Trip Logic & Trip indication & 1 & & & & & & 11 \\
\hline 77 & $T / G$ Control & Sw Panels & Manual Commands & 1 & & 2.10 & & & & 10 \\
\hline 78 & Trip Logic & Sw Panels & Master Trip & 1 & & 2.11 & & & & 7 \\
\hline 79 & ILS & Plant & ILS commands and data & 5 & & & & & 5.17 & 8 \\
\hline 80 & SCU & Plant & Data to SCU & 3 & & & & & 5.18 & 8 \\
\hline 81 & RLU & Plant & FLU commands and data & 2 & & & & & 5.19 & 8 \\
\hline 82 & DARMS & Plant & Llata to DARMS & 4 & 1.16 & & & & & 8 \\
\hline
\end{tabular}


Table 3-3. SIL Interface Crieckout Plan (Page 5 of 5)

*Remarks:

1. Disk Units shared by OCS and DAS computers.

2. Used in SIL only.

3. Data input from six wind towers and six sets of special heliostat instrumentation.

4. Data input from four weather stations and the circumsolar telescope.

5. BCS interfäces will be checked separate from the primary SIL using serrogate OCS computer.

6. HAC equipment must be simulated.

7. Trip lagic must be simulated.

8. Plant interface devices must be simulated.

9. Interface at DEC-10 probably not available during SIL time frame. Simulation may be required.

70. $T / G$ control equipment must be simulated.

11. Equipment not available for SIL; test at site. 

E. Fabricate Stage III SIL support equipment.
F. Prepare test control drawing for Stage VI.
G. Set up equipment test log books for Stage III.
H. Maintain equipment test log books.

\subsubsection{SIL Stage III Activities (SDPC Integration)}

The following activities will be performed during Stage III:

A. Receive, install, and checkout Stage III equipment as shown in Table 3-1.

B. Perform Stage III equipment additions/modifications as shown in Table 3-2.

C. Connect and check out Stage.III interfaces in accordance with Table 3-3.

D. Fabricate Stage IV cables.

E. Fabricate Stage IV SIL support equipment.

F. Set up equipment test log books for Stage IV.

G. Maintain equipment test log books .

\subsubsection{SIL Stage IV Actĩvities (Computer Update)}

The following activities will be performed during Stage IV:

A. Receive, install, and checkout Stage IV equipment as shown in Table 3-1.

B. Perform Stage IV equipment additions/modifications as shown in Table 3-2.

C. Connect and checkout Stage IV. interfaces in accordance with Table 3-3.

D. Fabricate Stage V cables.

E. Fabricate Stage V SIL support equipment.

$F$. Set up equipment test log books for Stage $V$.

G. Maintain equipment test log books.

\subsubsection{SIL Stage V Activities (SCU/RLU/ILS Integration)}

The following activities will be performed during Stage V:

A. Receive, install, and checkout Stage $V$ equipment as shown in Table 3-1.

B. Perform Stage $V$ equipment additions/modifications as shown in Table 3-2. 
C. Connect and checkout Stage $V$ interfaces in accordance with Table 3-3.

D. Release test control drawing for Stage VI.

E. Maintain equipment test log books.

\subsubsection{SIL Stage VI Activities (System Evaluation)}

The following activities will be performed during Stage VI:

A. Perform system-level functional tests identified in Table 3-4 to the extent possible within the scheduled time of SIL activities.

B. Prepare plan for Stage VII (packing and shipping).

C. Maintain equipment test log books.

D. Move BCS SIL control building equipment to maxi SIL area, integrate with OCS computer and checkout with software running under the Maxnet Operating System.

\subsubsection{SIL Stage VII Activities (Pack and Ship)}

The following activities will be performed during Stage VII:

A. Prepare control and instrumentation (C\&I) equipment for shipment to plant site.

B. Ship C\&I equipment to plant site.

C. Prepare SIL report.

\subsection{TEST CONFIGURATIONS}

\subsubsection{MVCU. Testing Configuration}

A Beckman multivariable control unit, which is the key element of the SDPC, will be installed in a laboratory area along with a Beckman Operator Interface Unit and terminating equipment. A minimum number of drivers will be fabricated to allow interfacing with the OLSF computer. Capability to manually input external discrete commands and analog functions and to measure responses will be provided.

\subsubsection{Test Hardware}

Figure 1-3 illustrates the configuration of the test hardware.

\subsubsection{Test Software}

Beckman "fill-in-the-blanks" firmware and operating procedures will be utilized to operate and configure the Beckman equipment. Somie software will 
Table 3-4. System Functional Tests (Page 1 of 2)

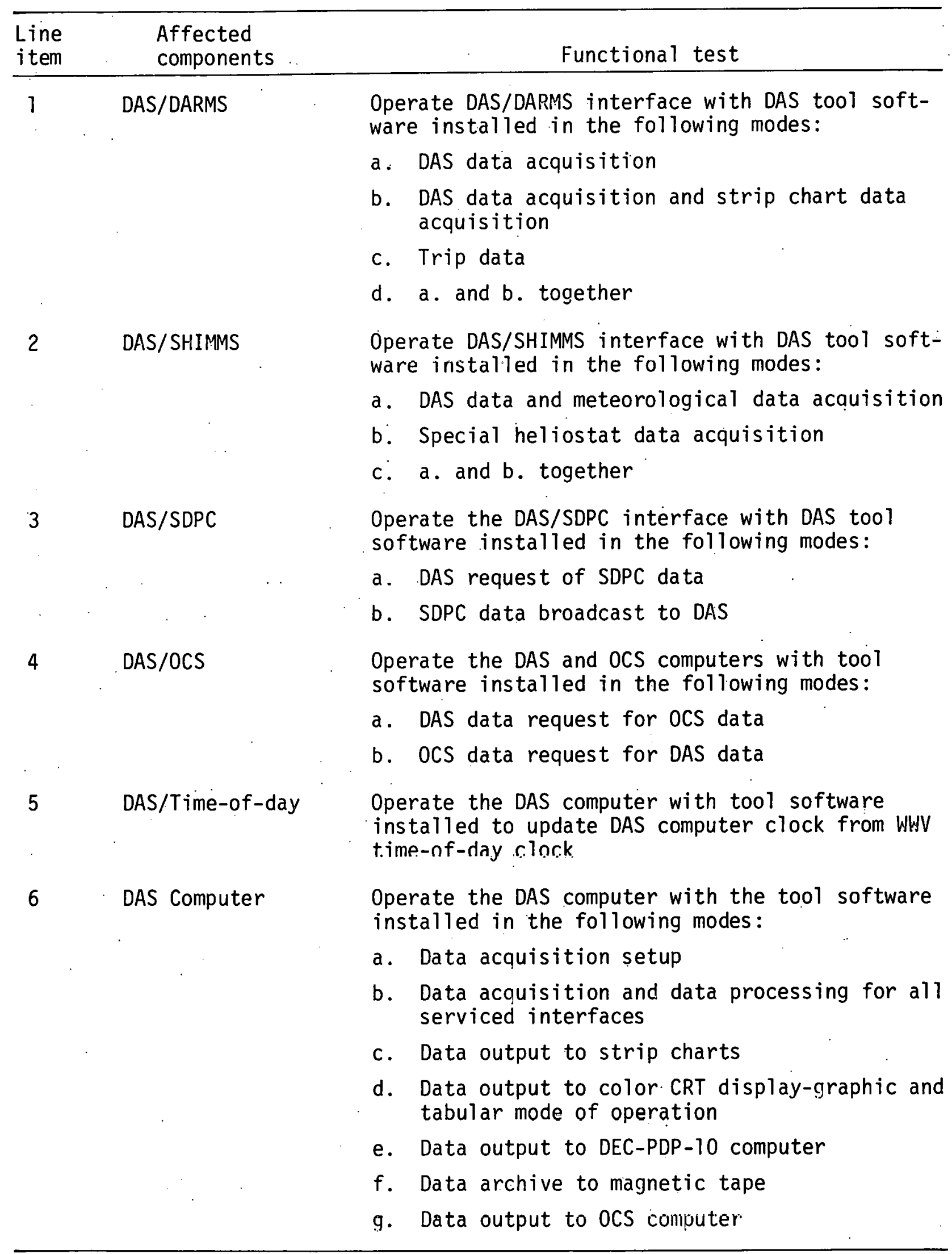


Table 3-4. System Functional Tests (Page 2 of 2)

\begin{tabular}{|c|c|c|}
\hline $\begin{array}{l}\text { Line. } \\
\text { item }\end{array}$ & $\begin{array}{l}\text { Affected } \\
\text { components }\end{array}$ & Functional test \\
\hline \multirow[t]{2}{*}{6} & $\begin{array}{l}\text { DAS Computer } \\
\text { (cont.) }\end{array}$ & $\begin{array}{l}\text { h. a. through g. coordinated to simulate } \\
\text { typical site operation }\end{array}$ \\
\hline & & i. DAS computer diagnostics \\
\hline \multirow[t]{4}{*}{7} & $\begin{array}{l}\text { OCS/OCS Graphics } \\
\text { (See Note 1) }\end{array}$ & $\begin{array}{l}\text { Operate the OCS computer with the OCS graphic } \\
\text { terminal interface software installed in the } \\
\text { following modes: }\end{array}$ \\
\hline & & a. Graphic picture building \\
\hline & & $\begin{array}{l}\text { b. Graphic picture display of background with } \\
\text { simulated dynamic data. }\end{array}$ \\
\hline & & $\begin{array}{l}\text { c. Graphic picture select from keyboard for one } \\
\text { and two display terminals }\end{array}$ \\
\hline \multirow[t]{4}{*}{8} & $\begin{array}{l}\text { OCS/DAS/SDPC } \\
\text { (See Note 1) }\end{array}$ & $\begin{array}{l}\text { Operate the OCS computer with oCS tool software } \\
\text { that acquires data to be displayed on the OCS } \\
\text { graphics terminal in the following modes: }\end{array}$ \\
\hline & & a. SDPC data acquire and display \\
\hline & & b. DAS computer data acquire and display \\
\hline & & $\begin{array}{l}\text { c. DAS/SDPC data acquire concurrently and } \\
\text { display }\end{array}$ \\
\hline \multirow[t]{6}{*}{9} & SDPC/ILS & $\begin{array}{l}\text { Operate the SDPC with the ILS connected in the } \\
\text { following modes: }\end{array}$ \\
\hline & & a. Configure SDPC for plant start up \\
\hline & & b. Configure ILS for plant start up \\
\hline & & c. Build graphic display on SDPC terminal \\
\hline & & $\begin{array}{l}\text { d. Operate from the SDPC console with selected } \\
\text { simulated inputs from the SDPC and ILS remote } \\
\text { hardware. }\end{array}$ \\
\hline & & e. Display selected inputs on graphic display \\
\hline \multirow[t]{2}{*}{10} & $\mathrm{SDPC} / \mathrm{ILS} / \mathrm{SCU} / \mathrm{RLU}$ & a. Simulate a plant trip initiated from the RLU \\
\hline & & $\begin{array}{l}\text { b. Operate from the SUPC console with selected } \\
\text { simulated inputs from the SCU }\end{array}$ \\
\hline
\end{tabular}


be provided to be resident in the OLSF computer to simulate responses to the MVCU.

\subsubsection{Maximum SIL Test Configuration}

The maximum SIL test configuration will include as many elements of the Pilot Plant controls as are available. Figure 3-9 is an overview of the planned maximum SIL test configuration, and each major component will be tested for readiness prior to interfacing and then integrated to support system testings. The Master Control Console houses many elements from different components and should be the central operating point for all SIL activities.

\subsubsection{Operational Control System}

\section{Hardware}

The OCS hardware to be tested is illustrated in Figure 3-10. The MODCOMP computer and its peripherals and the color CRT terminals make up this component. Figures 3-11 and 3-12 are a block diagram of OCS computer and Figure 3-13 is a block diagram of the color CRT terminals. MODCOMP diagnostics shall be utilized to demonstrate MODCOMP hardware readiness. Test support software shall be utilized to demonstrate the internal interface between the MODCOMP and the color CRT terminals.

\section{OCS Software Configuration}

The following paragraphs discuss the software configuration that will be used to accomplish the interfacing of computer vendor supplied peripherals and peripherals supplied by subcontractors to the SFDI.

The OCS computer system will be delivered to the SIL in an initial configuration.

An additional set of peripheral equipment will be provided, as indicated by the schedule in Figure 3-6, to bring the OCS to the final as-delivered configuration midway through the SIL testing period. 


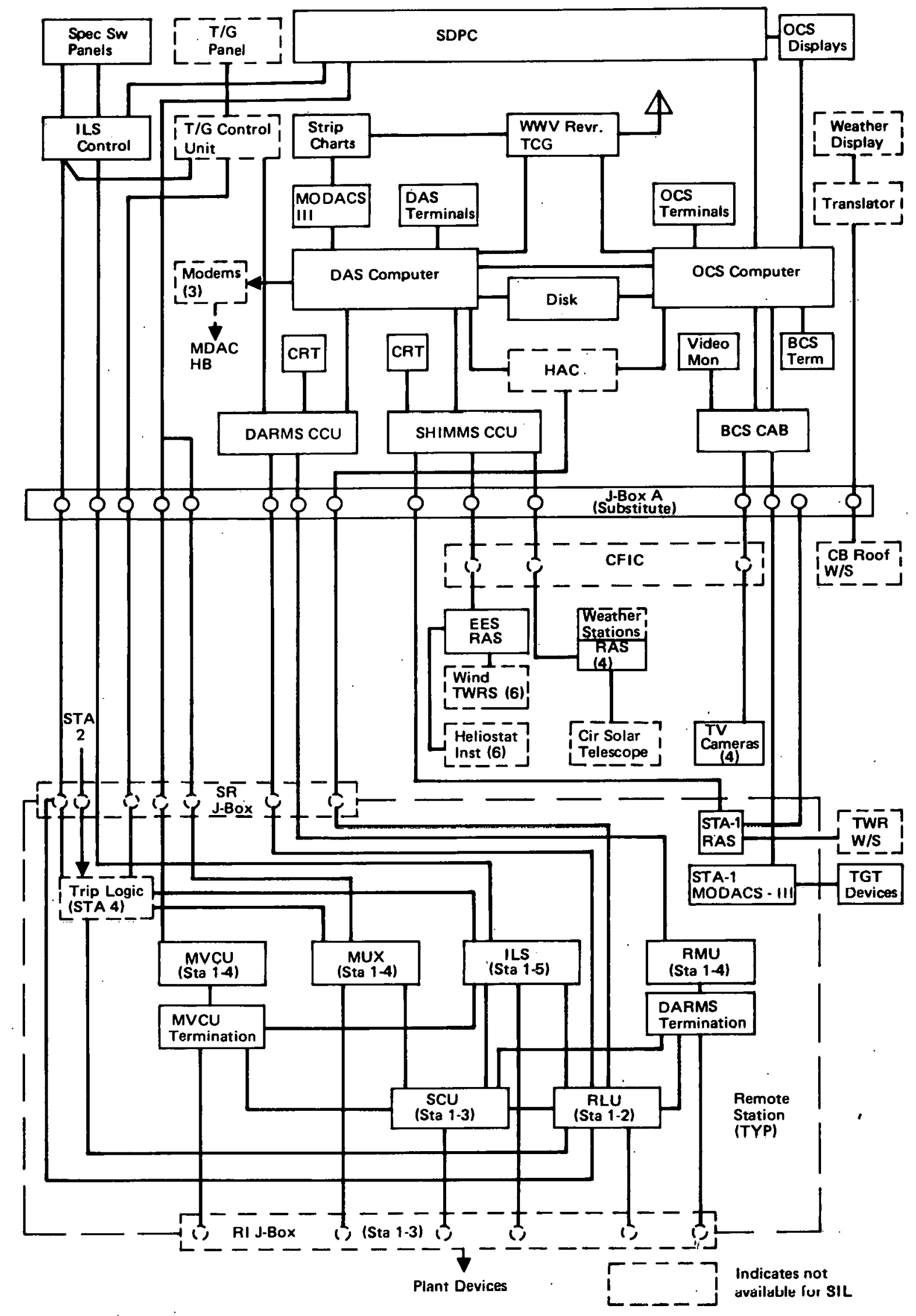

Figure 3-9. Maxi SIL Test Configuration 


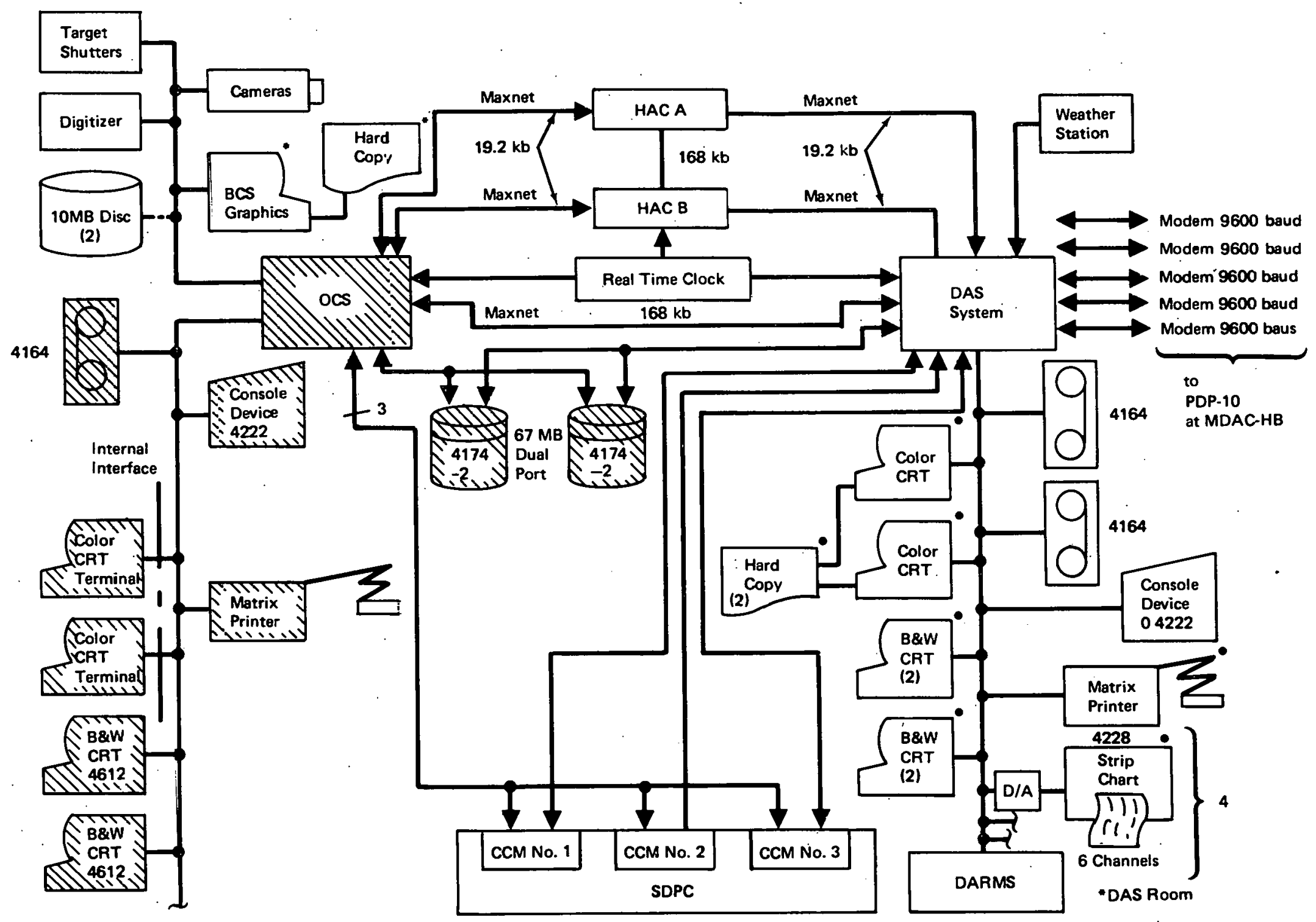

Figure 3-10. Ocs Computer Configuration 


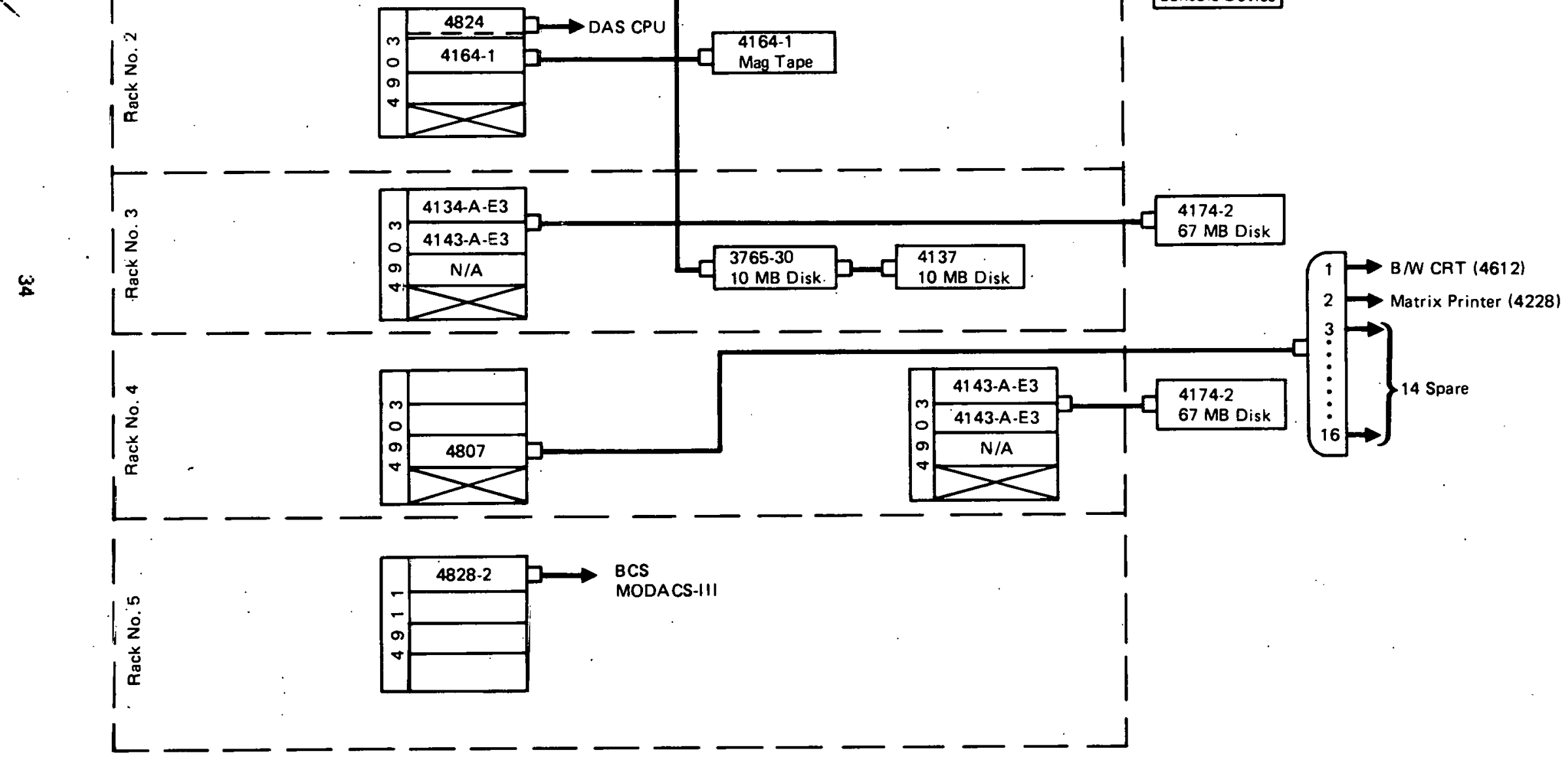

Figure 3-11. OCS Computer Block Diagram (Initial Configuration) 

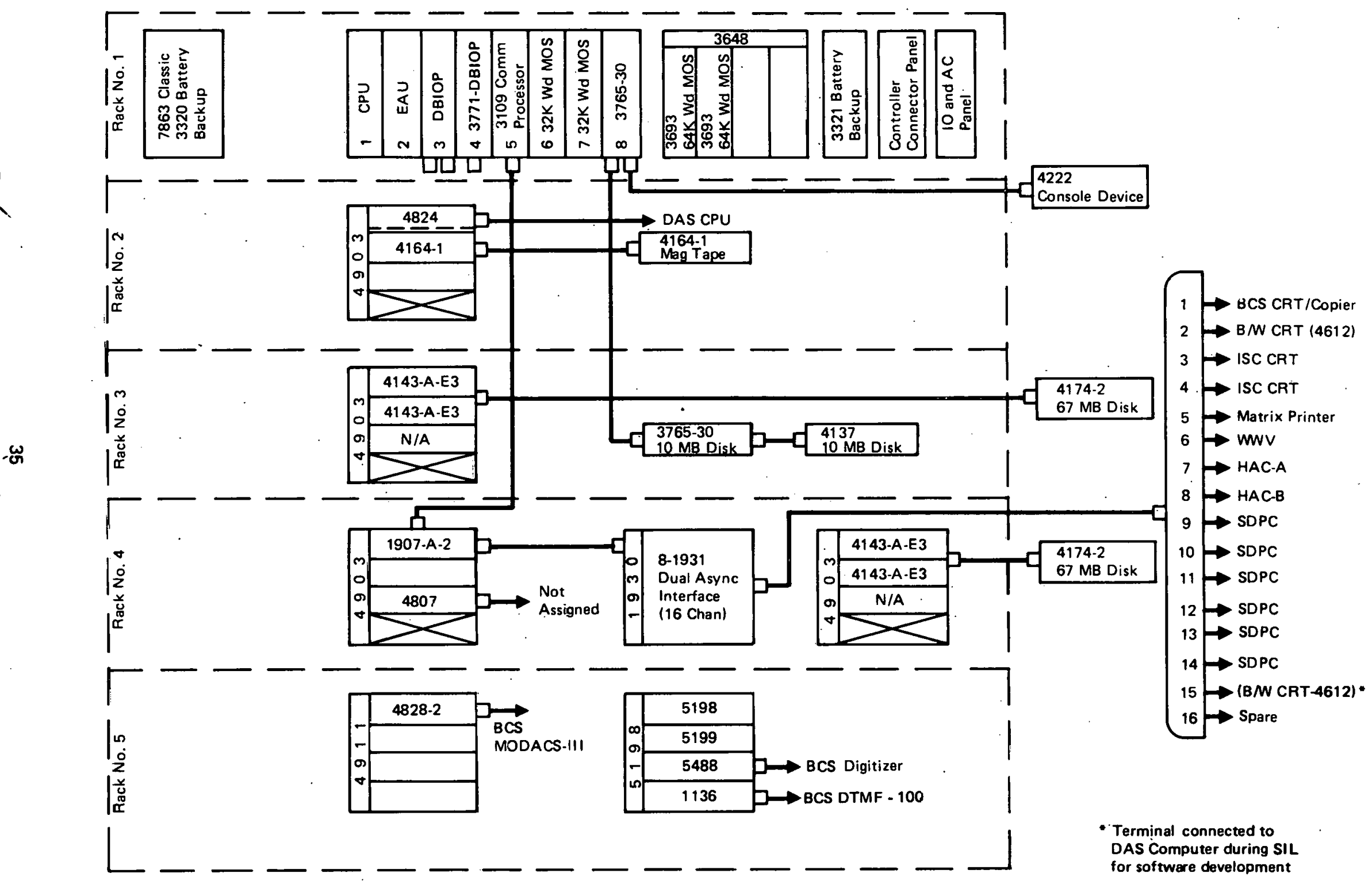

Figure 3-12. OCs Computer Block Diagram (Operational Configuration) 


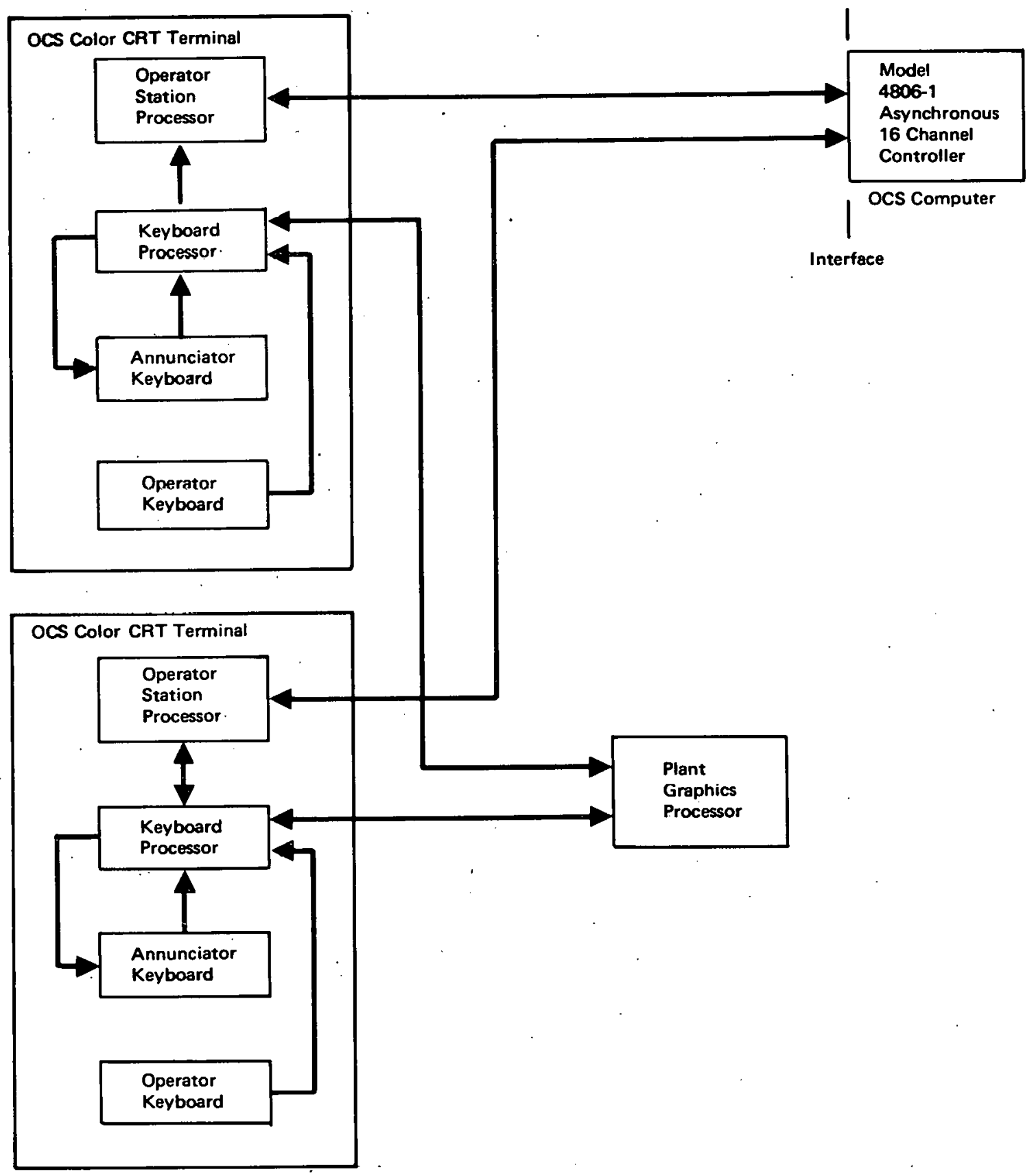

Figure 3-13. OCS Color CRT Terminal Block Diagram 
MODCOMP Peripheral Equipment. When the OCS computer system is delivered in its initial configuration, the following MODCOMP supplied peripheral devices will be tested by MODCOMP field installation personnel using standard MODCOMP diagnostic programs.
A. OCS tape drive, 9 track, 800 BPI, 75 IPS
B. Console device (keyboard/printer)
C. $67 \mathrm{MB}$ disc drives (dual ports)
D. $10 \mathrm{MB}$ disc drives

WWV. This peripheral device is designed to generate electrical signals which represent coordinated universal time information in a coded format. Operational receiving and decoding. software will be exercised to verify that the WWV signals are being received by the OCS.

A special test software module will be required to continuously monitor the output of the WWV decode module and display that output to a CRT in conventional time format, i.e., HH:MM:SS.

OCS Final Configuration. When MODCOMP installs the additional peripherals to the OCS computer system, the following devices will be installed and tested by MODCOMP field personnel:

A. The MAXNET IV interface between the OCS and DAS computer systems. MODCOMP will verify that the communications link between the two computers is operational and that messages can be sent from one application program to another.

B. The matrix printer will be installed and verified by MODCOMP personnel using standard diagnostic test programs supplied by MODCOMP.

C. Two additional black and white CRT terminals will be connected to the OCS. MODCOMP personinel will test the terminals using standard MODCOMP test diagnostic software.

Color CRTs. Two special Beckman supplied color CRTs will be interfaced to the OCS after the OCS is equipped with all MODCOMP supplied peripherals. 
These special CRTs will have to be tested in several stages which are described below:

A. It is expected that the interface (I/F) to these CRTs will be a standard I/F corresponding to RS-232-C; therefore, a standard software driver can be used to perform the physical tasks of testing status, performing the necessary "are-you-ready?" tests and moving messages across the I/F.

B. The message content will be verified by designing and building special test software to invoke the standard driver to send or receive "canned" messages to/from the color CRT.

The test software must be designed to permit a test operator to select which "canned" message is to be sent via the system support software from one of the black and white terminals. This special piece of test software plus the debugging capability of the MODCOMP system will allow a preselected set of messages to be sent to and received from the CRTs. This set of messages will test all operational capabilities of the CRTs.

SDPC. The OCS interfaces with the Beckman SDPC in two ways. The first is a connection to the SDPC Host Communications Processor (HCP) and to the Peripheral Interface Processor (PIP). See Figure 3-14. Interfaces to the HCP are used to read data from the CCM and write new data information into the CCM.

Interfaces to the PIP are used to collect data on a periodic basis from the OCS. In this usage, the OCS will be simulating a MVCU which allows the OCS to provide calculated parameters to the SDPC as if those parameters were collected from the field.

Reading Data from CCM. The first step in checking out the OCS/SDPC interface is to load a CCM with hexadecimal data via the SDPC debugging program. This provides a known reference from which a comparison can be made. once the data is read over to the OCS.

The next step is to enable the MODCOMP debugging program and allow the HCP read program to execute once in the OCS and return to the debug monitor. When this happens, the data buffer that was read can be dumped to either a CRT or printer where it can be verified that the data read is indeed the data that is expected. 


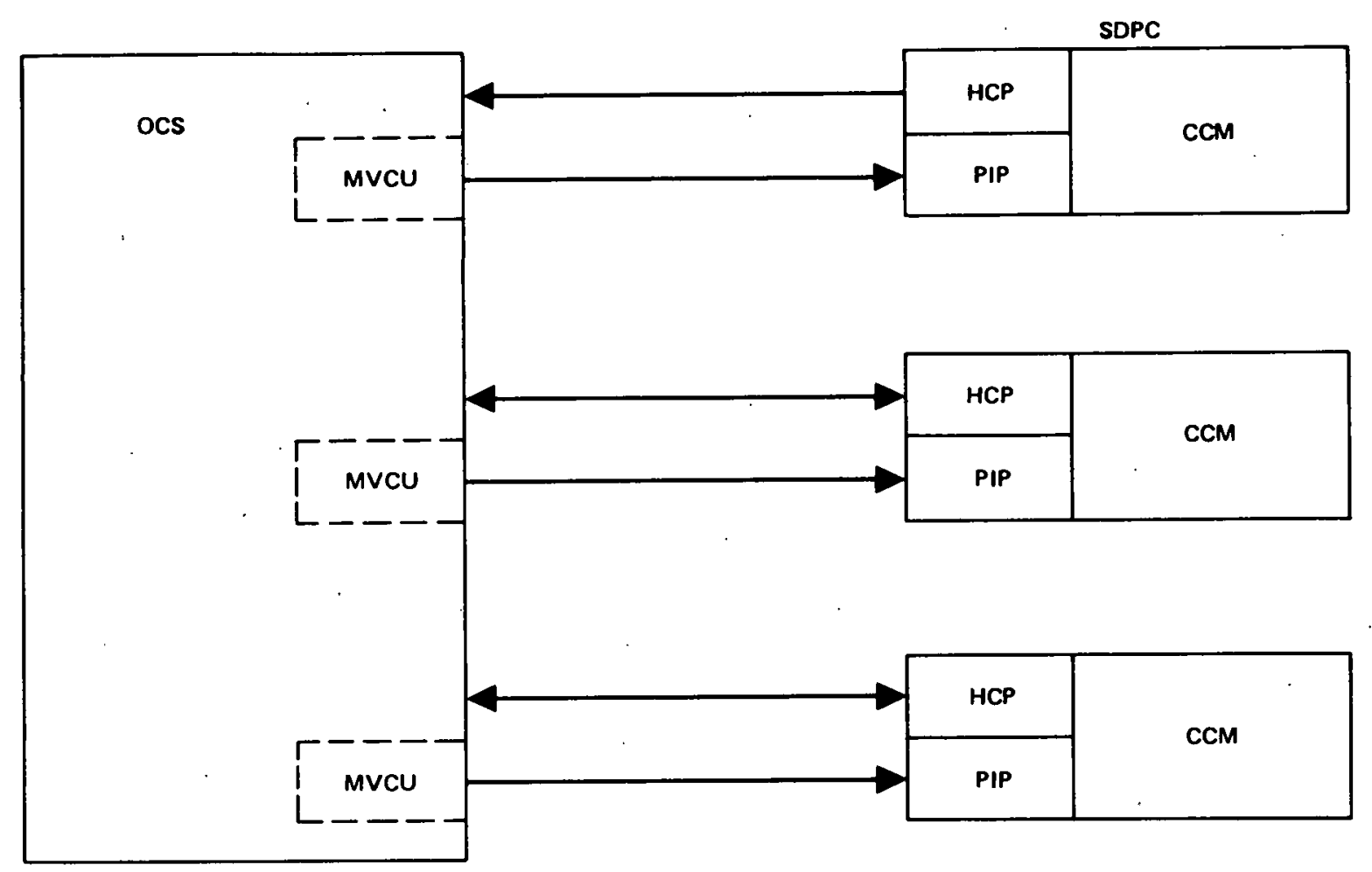

Figure 3-14. OCS/SDPC Interface

In a similar way, data can be structured in the OCS data buffer and sent to the HCP and deposited into the CCM and the SDPC debug program can verify that the CCM did receive the data in the proper form.

Data transfer between the OCS and PIP/CCMs can be verified in the same manner as described above.

\subsubsection{Data Acquisition System Configuration}

\section{Hardware}

The DAS hardware to be tested is illustrated in Figure 3-15. The MODCOMP computer and its peripherals, the color CRT terminals, the strip chart recorders, the data acquisition and remule multiplexer system and the hard copier make up this component. Figures 3-16. and 3-17 are detailed block diagrams of the DAS computer, and Figure 3-18 is a block diagram of DARMS. Figure 3-19 is a block diagram of the color CRT terminals. 


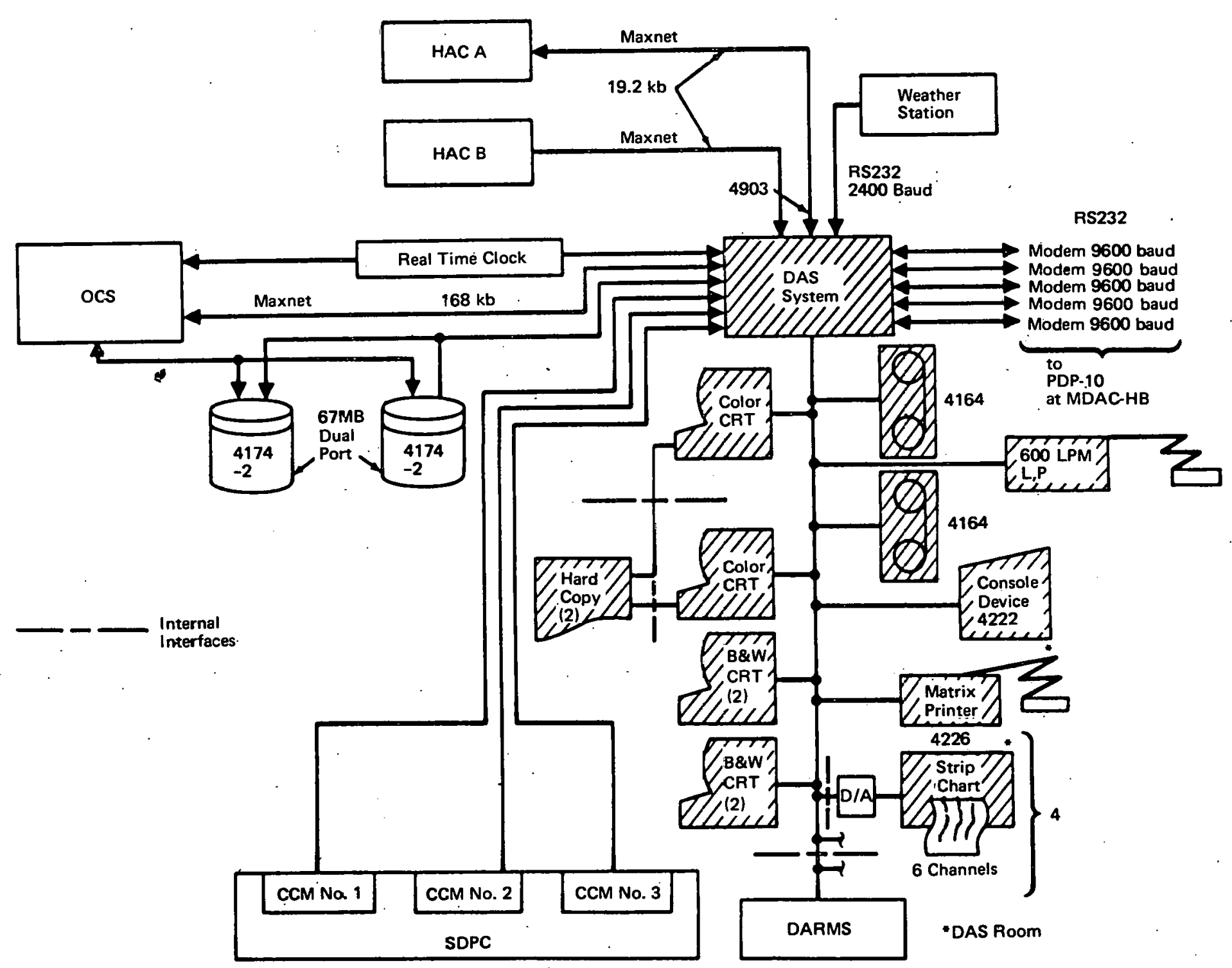

Figure 3-15. DAS Configuration 


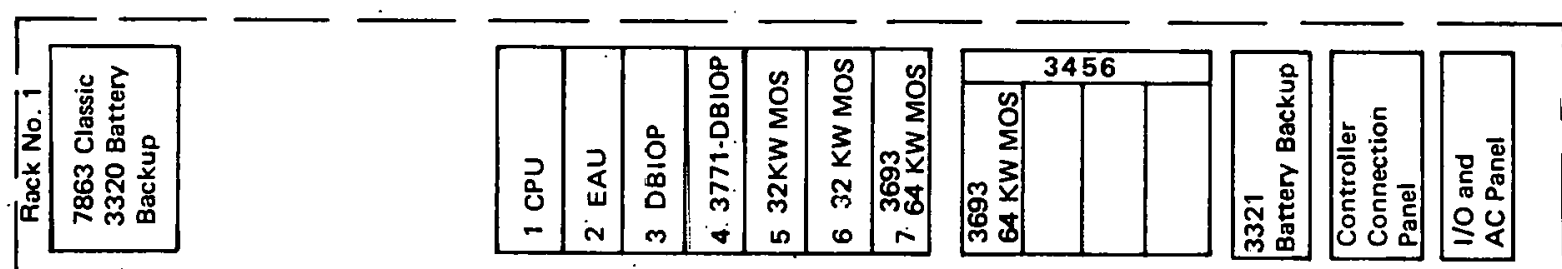

\section{(8)}

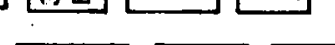

$\pm$<smiles>C(=CC1CC1)C1CC1</smiles>

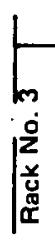

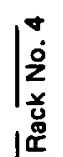

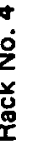

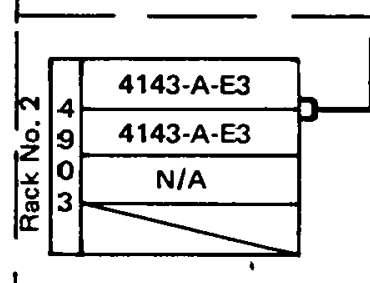

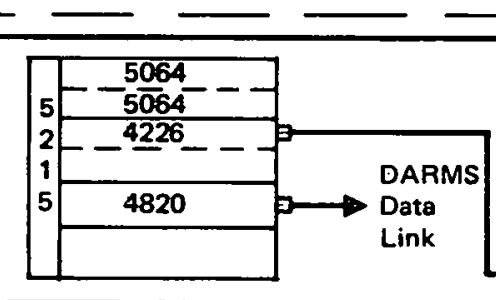

.
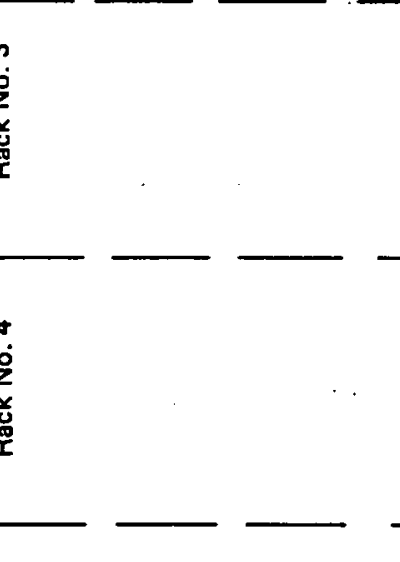

$\longrightarrow$

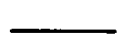

ink
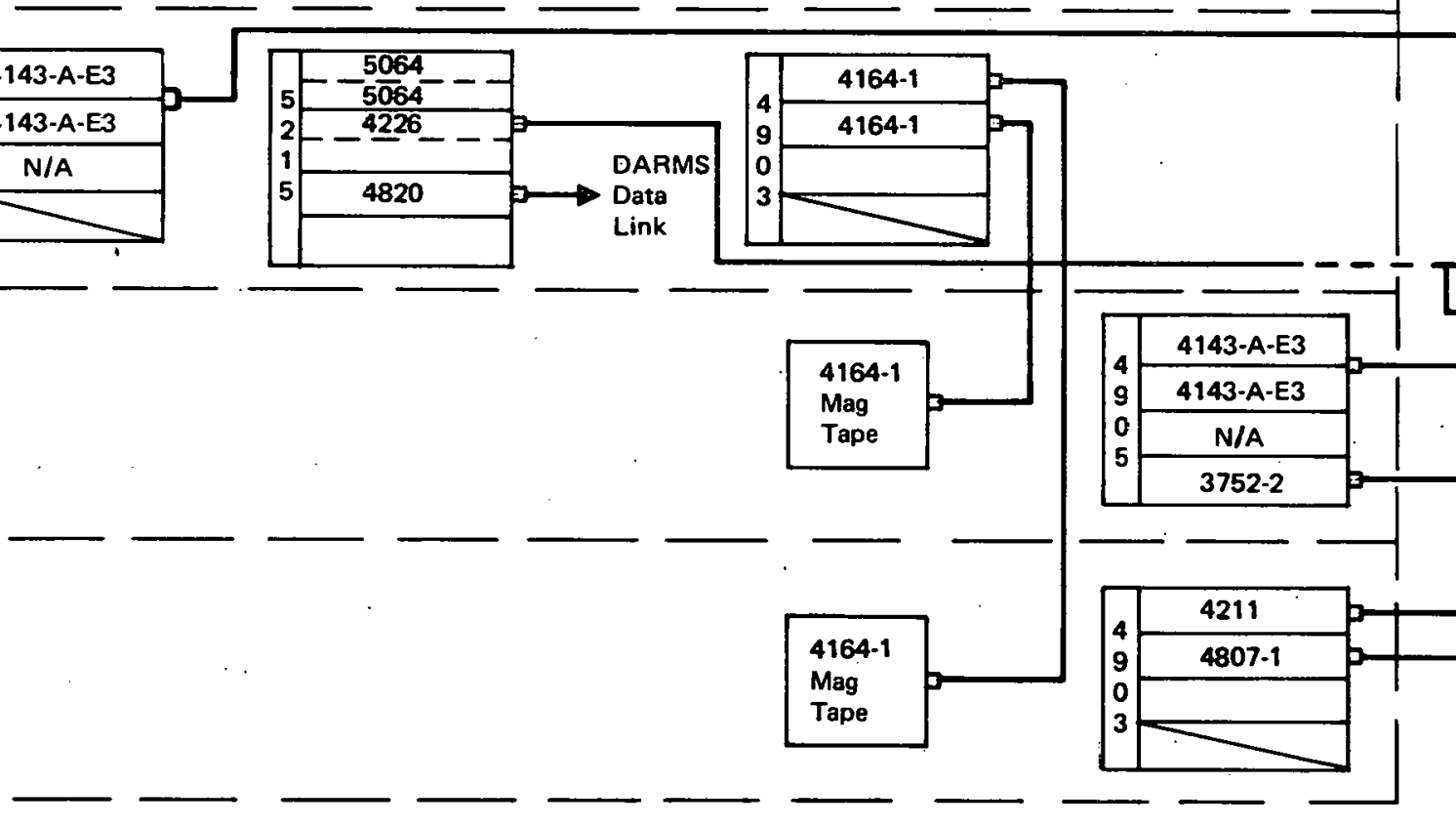

4174-2

67-MB Disk
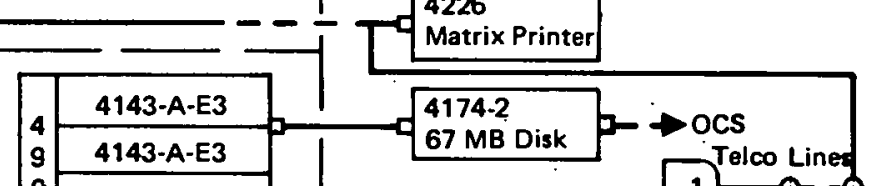

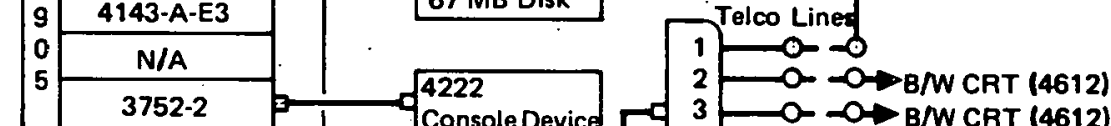

Console Device $[\mathrm{C}$ 3 $\rightarrow$ B/W CRT (4612)

B $\rightarrow$ BN CRT (4612)

6 B 1 BW CRT (4612)

$\sqrt{4211600}$

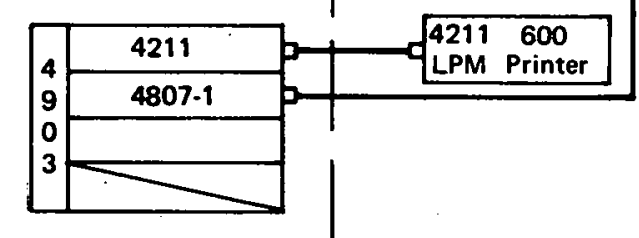

B/W CRT (4612)

$8 \longrightarrow$ Color CRT/Printer

$9 \longrightarrow$ Color CRT/Printor

$10 \longrightarrow$ WWV

$11 \longrightarrow$ DARMS CMD, Link

12

5 Spares

Figure 3-16. DAS Computer Block Diagram (Initial Configuration) 


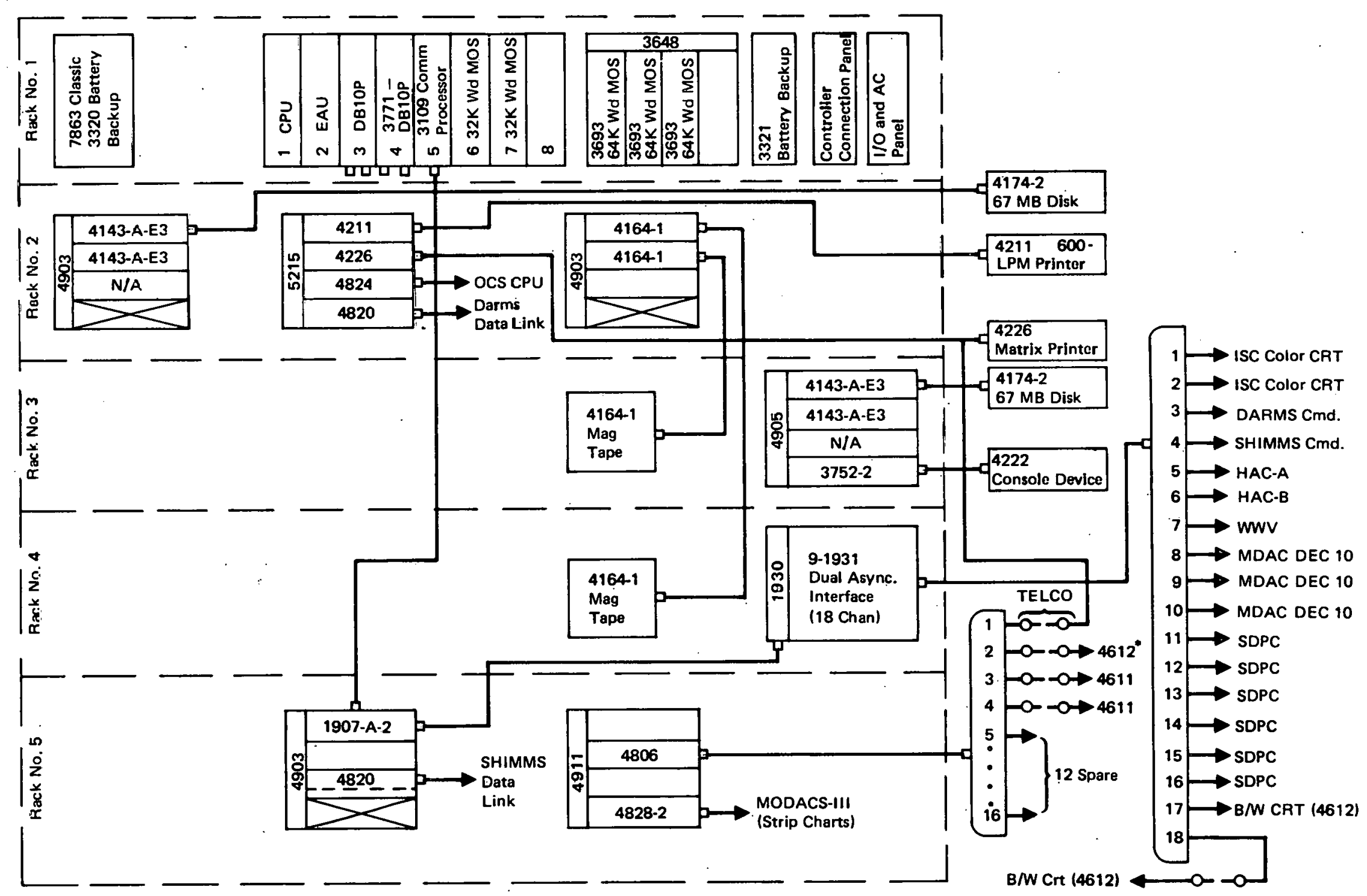

- Part of OCS Computer. Temporarily on DAS for Software Development

Figure 3-17. DAS Computer Block Diagram (Operational Configuration) 

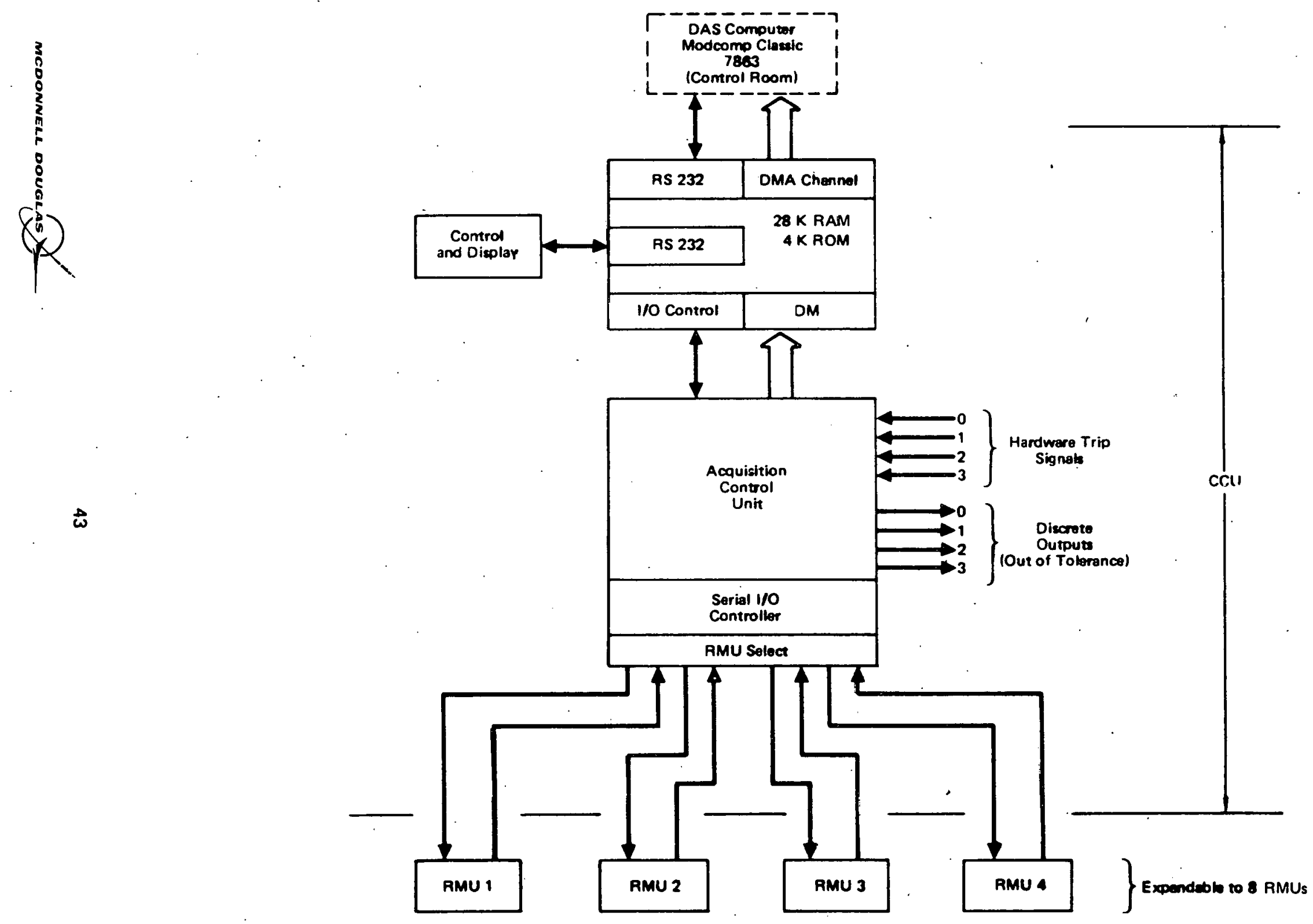

Figure 3-18. Data Aequisition Remote Multiplexer System 


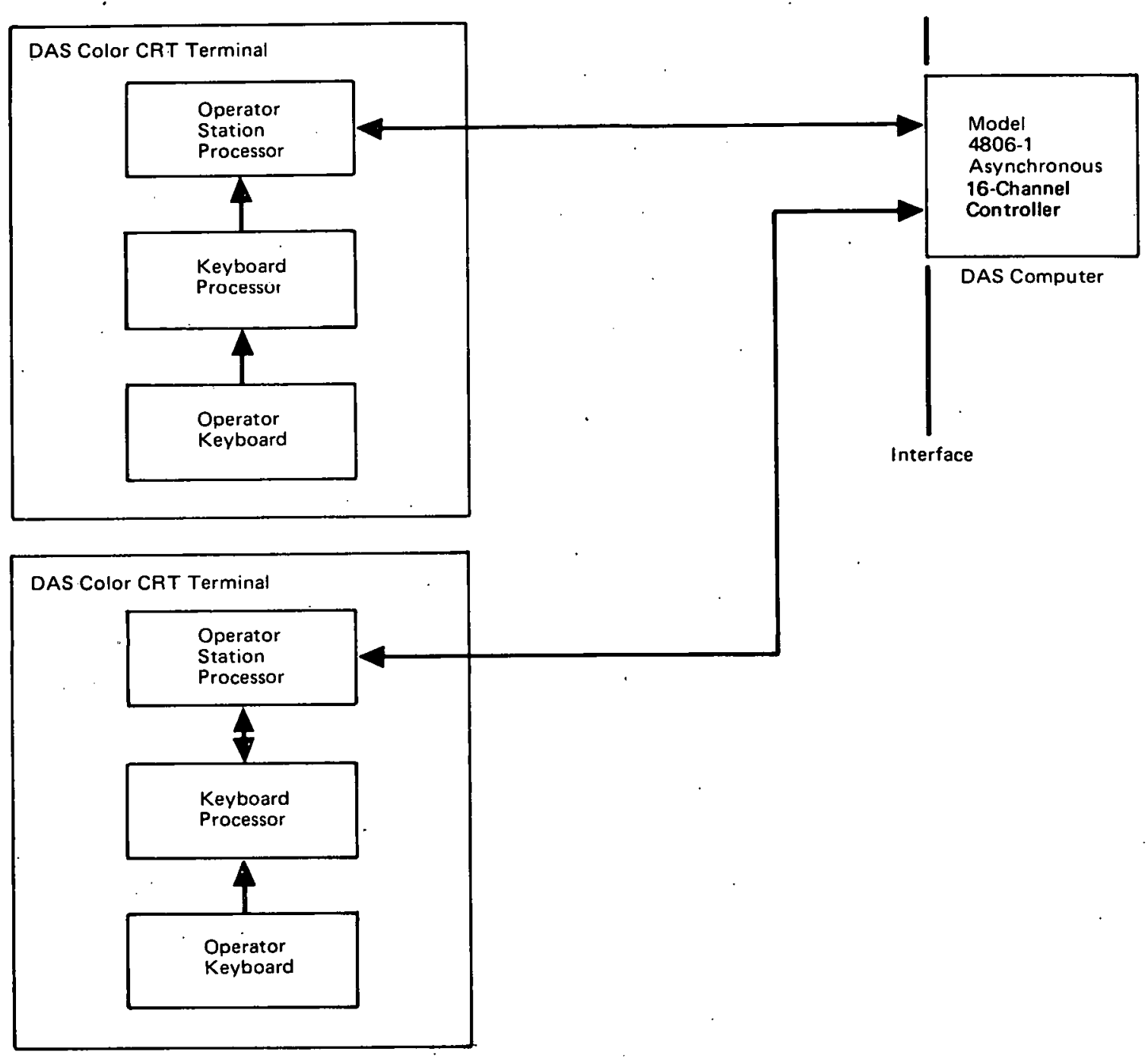

Figure 3-19. DAS Color CRT Terminal Block Diagram 
MODCOMP software diagnostics will be used to demonstrate MODCOMP computer hardware readiness. The DARMS will use a diagnostic program provided in firmware by Cyber to determine readiness. Test support software will be utilized to demonstrate the internal interfaces between the MODCOMP and the color CRT terminals, the DARMS, the hard copier, and the strip chart recorders. .

\section{Software}

Software is shown in Figure 3-20.

A. Interface Verifications

1. DARMS-DAS Interface

a. Command Link - RS-232 - Standard software will be used to download known data to DARMS. The DARMS will perform balance and calibration operations and transmit calibration coefficients to DAS.

b. Data Link - The software interface will be verified by proper acquisition of data per standard software. Selected data channels will be stimulated to provide known data outputs.

c. High-Speed Scan Interrupt - A simulated trip condition to the DARMS will be verified to initiate collection of high-speed data scan via SFDI developed software.

\section{SHIMMS-DAS Interface}

a. Command Link - Requirements identical to DARMS-DAS interface except that the software utilized will be for SHIMMS instead of DARMS.

b. Data Link - Requirements identical to DARMS-DAS interface except that the SHIMMS acquisition program will be used.

3. WWV-DAS - The driver developed for the OCS computer will be used to show that DAS computer time can be synchronized to WWV time using special test software module.

4. SDPC-DAS Data Link - SFDI developed software will be used to acquire data from the SDPC. Acquired data will be analyzed using special test software to verify correct operation of the communication protocol and the software interfaces.

5. DAS to MODACS III - A special driver will be developed to down load MODACS III with applications software and to transmit canned data to MODACS III. Expected strip chart responses will constitute verification of the software interfaces. 

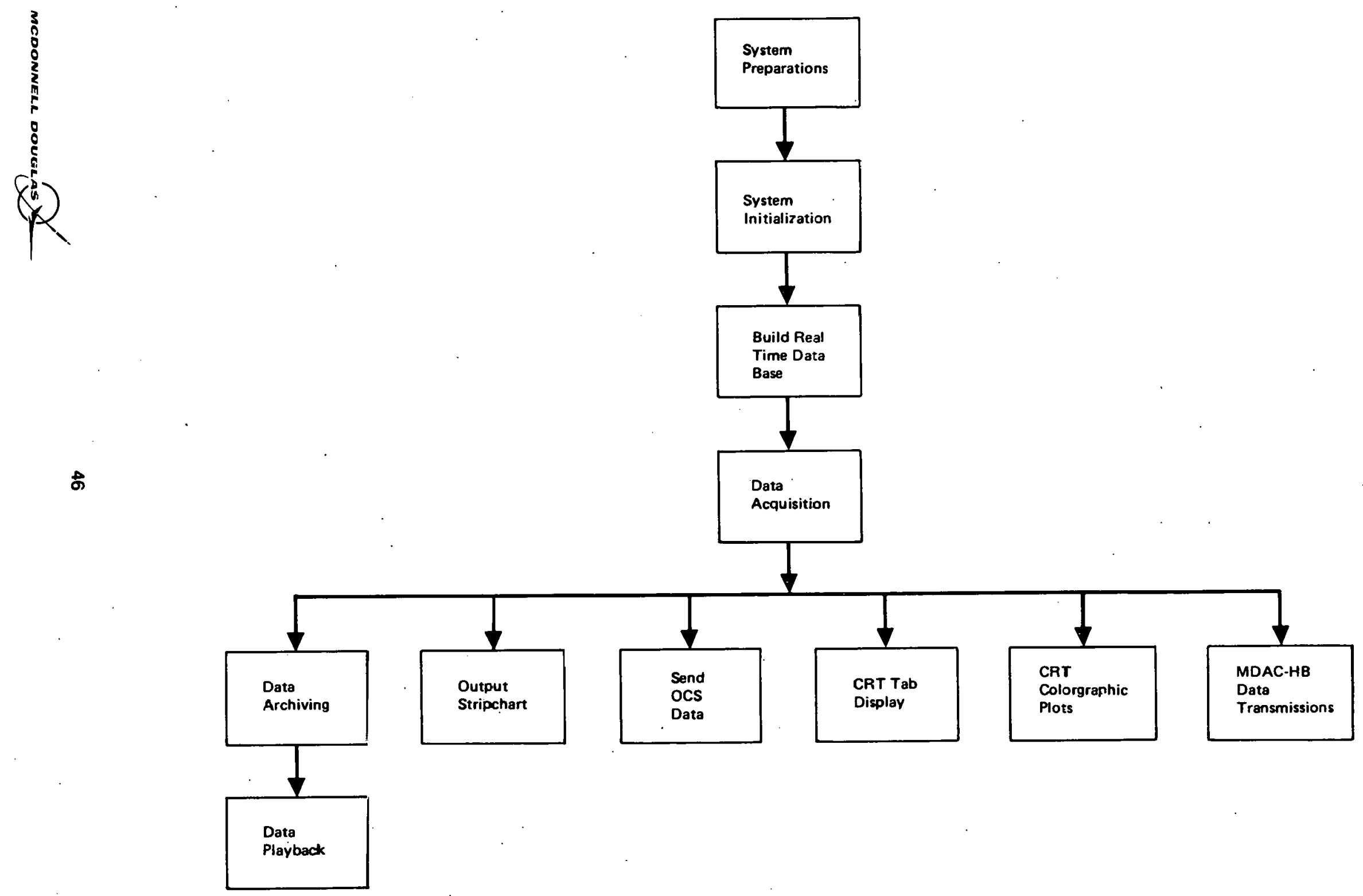

Figure 3-20. DAS Softwarz Hierarchy Chart, Major Functions 
6. DAS to MDAC-DEC 10 - Special driver will be developed to control SF.DI developed software used to transmit data via phone lines to test hardware thatwill verify DAS software interfaces.

7. DAS to HAC - Special driver shall be developed to simulate the HAC. A terminal shall be used as anI/0 device to check out SFDI developed HAC acquisition software.

8. DAS to OCS - Special driver will be developed to control standard software that will furnish or retrieve OCS/DAS status data or process OCS/DAS data requests via MAXNET core devices.

Data Processing

B. Data Archiving - SFDI developed software will be exercised to verify that data from external sources can be archived on magnetic tape and that disk files can be archieved to tape.

C. Data Base Construction - SFDI developed software will be exercised to verify that the real time data base can be constructed and maintained by DAS.

D. CRT Format and Tabulate Data - SFDI developed software will be exercised to verify that data residing in the real time data base can be displayed on the color CRTs.

E. CRT Format and Plot - SFDI developed software will be used to demonstrate that data residing in the real time data base can be displayed on the color CRTs.

F. Data Playback

1. Trip Data Processing - SFDI developed software will be exercised to demonstrate that trip data residing on diș can be processed to yield a sequence of events line printer output.

2. Normal Playback - SFDI developed software will be exercised to demosntrate that data residuing on tape can be played back for display on DAST. CRTs .

G. Operator Control Interface - SFDI developed software will be exercised to demonstrate that operator can initiate tasks and perform various DAS functions.

H. DAS System Test - DAS system operation from startup thru wrapup will be demonstrated using SFDI developed software.

\subsubsection{Subsystem Distributed Process Control}


Hardware

The SDPC hardware to be tested, illustrated in Figure 3-21, will be centrally located in the Master Control. Console Equipment illustrated in Figures 3-22 through 3-25 which will be located at remote stations and interconnected by data highways to the equipment in the master control console. The major elements of the SDPC are the color CRT terminals, the strip chart recorders, the printer/loggers, interlock logic, remote multivariable controllers, IPAC multiplexers and interlock logic 1/0. Beckmann "fill-in-theblank" firmware will be used to configure the SDPC and demonstrate hardware readiness.

\section{Software}

No SDPC software is required.

\subsubsection{Real-Time Clock}

Hardware

A real-time clock located in the master control console will be verified with the WWV/time code generator supplied for OCS and DAS computers.

\section{Software}

No software is required.

\subsubsection{Signal Conditioning Unit}

Hardware

Representative signal conditioning units for the Receiver Subsystem and Thermal Storage Subsystem to be tested will be supplied by Rocketdyne.

\section{Software}

No Signal Conditioning Unit software is required.

\subsubsection{Red Line Unit}




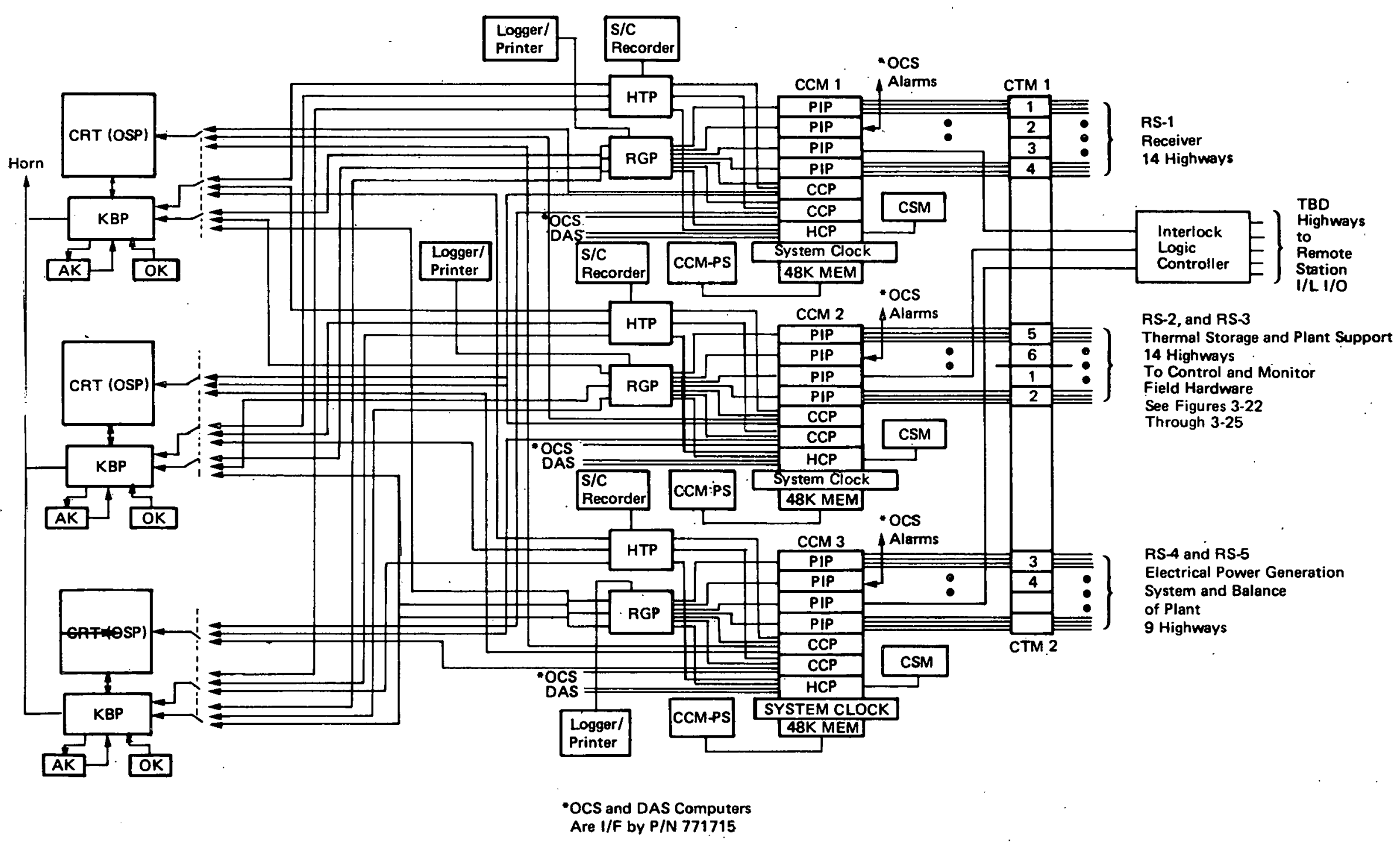

Figure 3-21. SDPC Test Configuration (Equipment Installed in Master Control Console P/N 610617) 


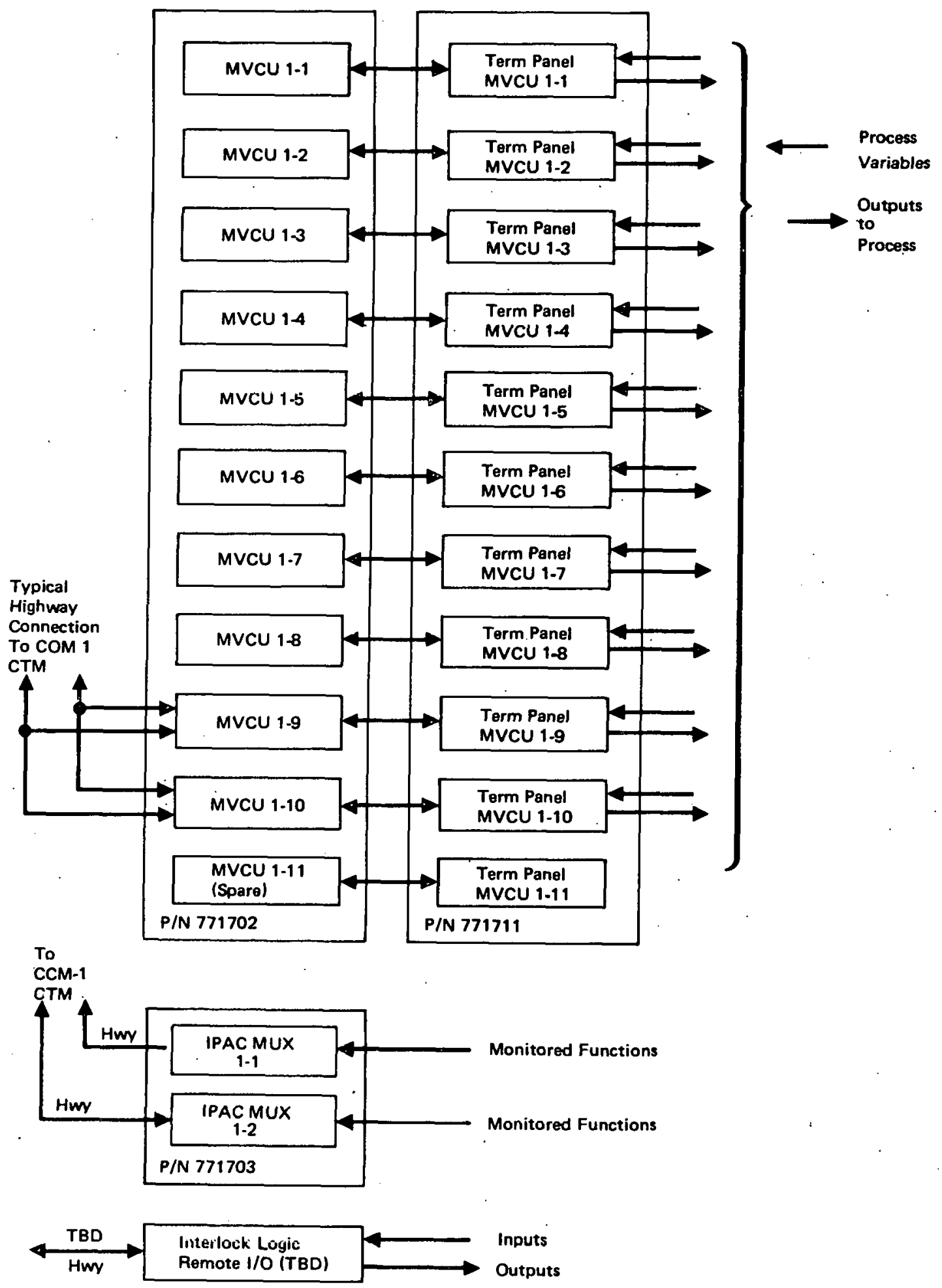

Figure 3-22. SDPC Remote Station - 1 

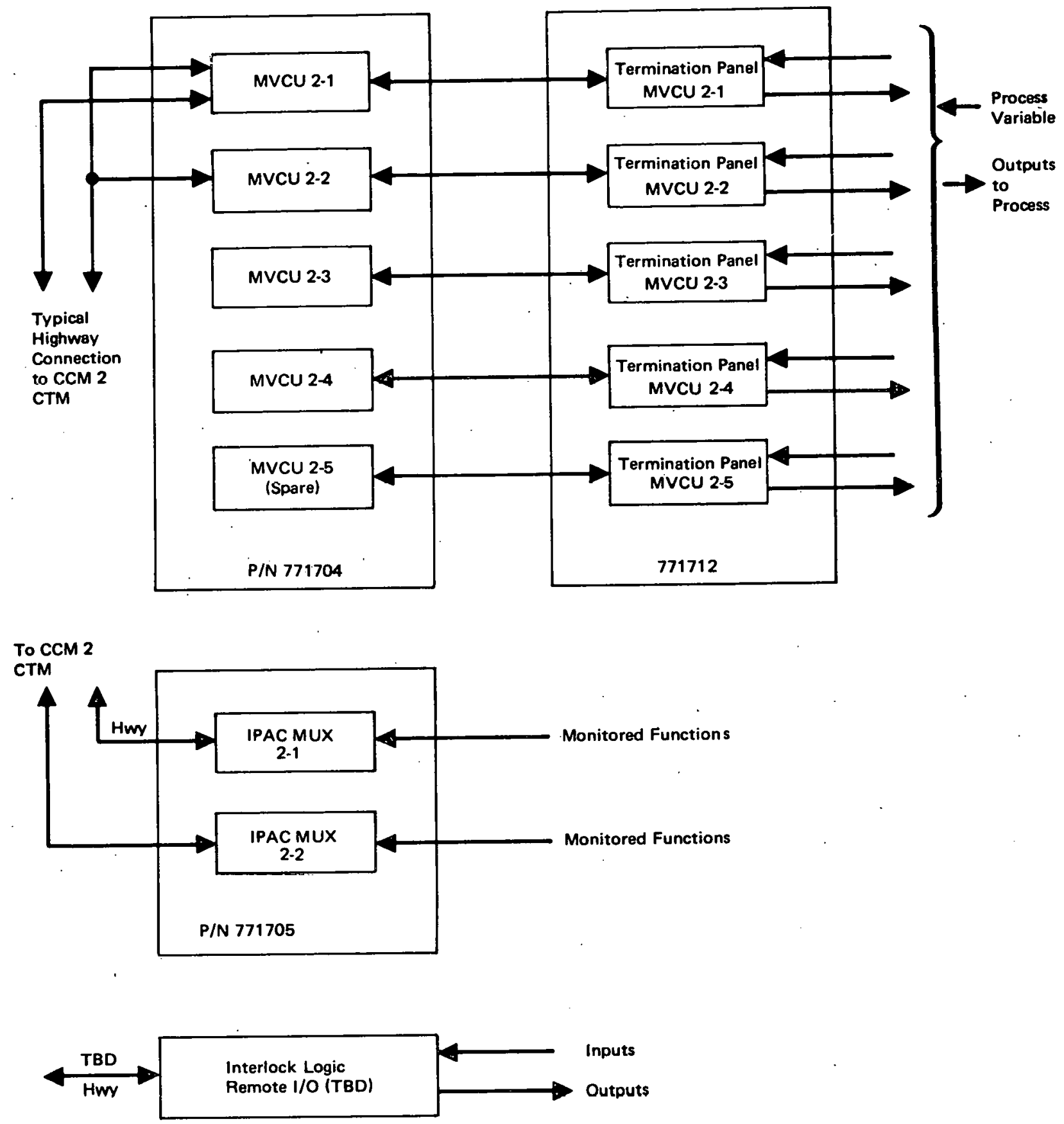

Figure 3-23. SDPC Remote Station - 2 

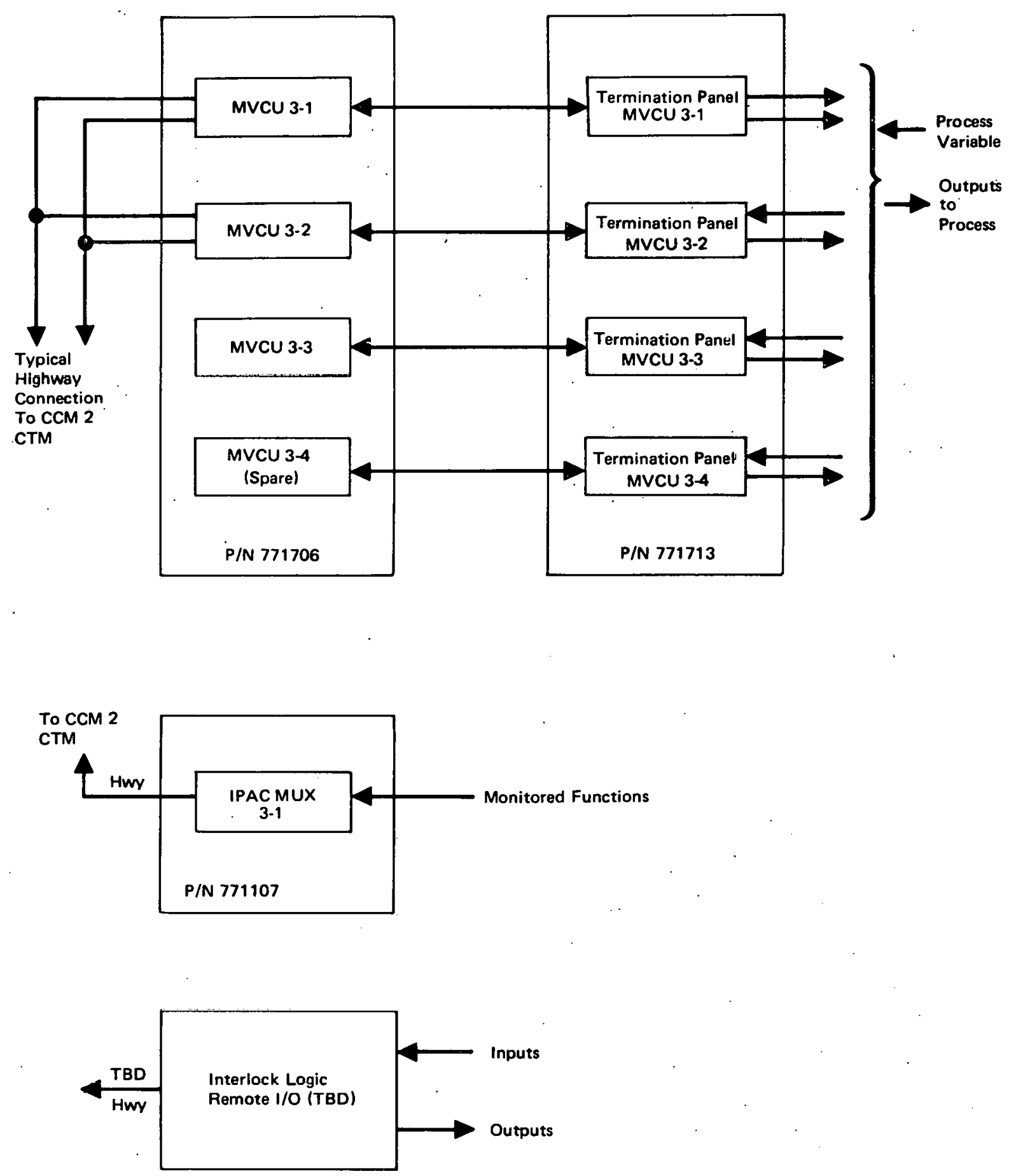

Figure 3-24. SDPC Remote Station - 3 


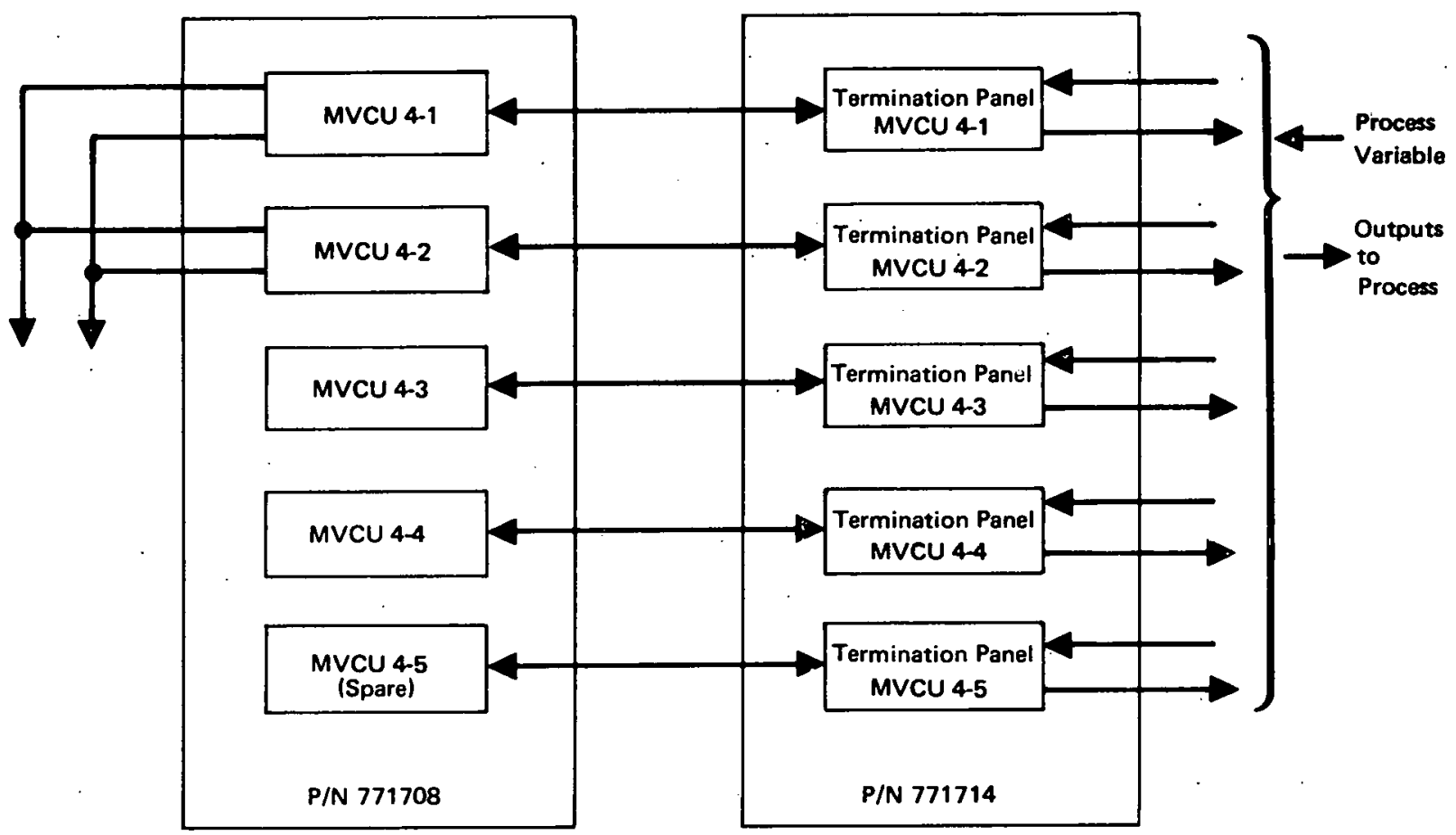

To CCM 3

CTM
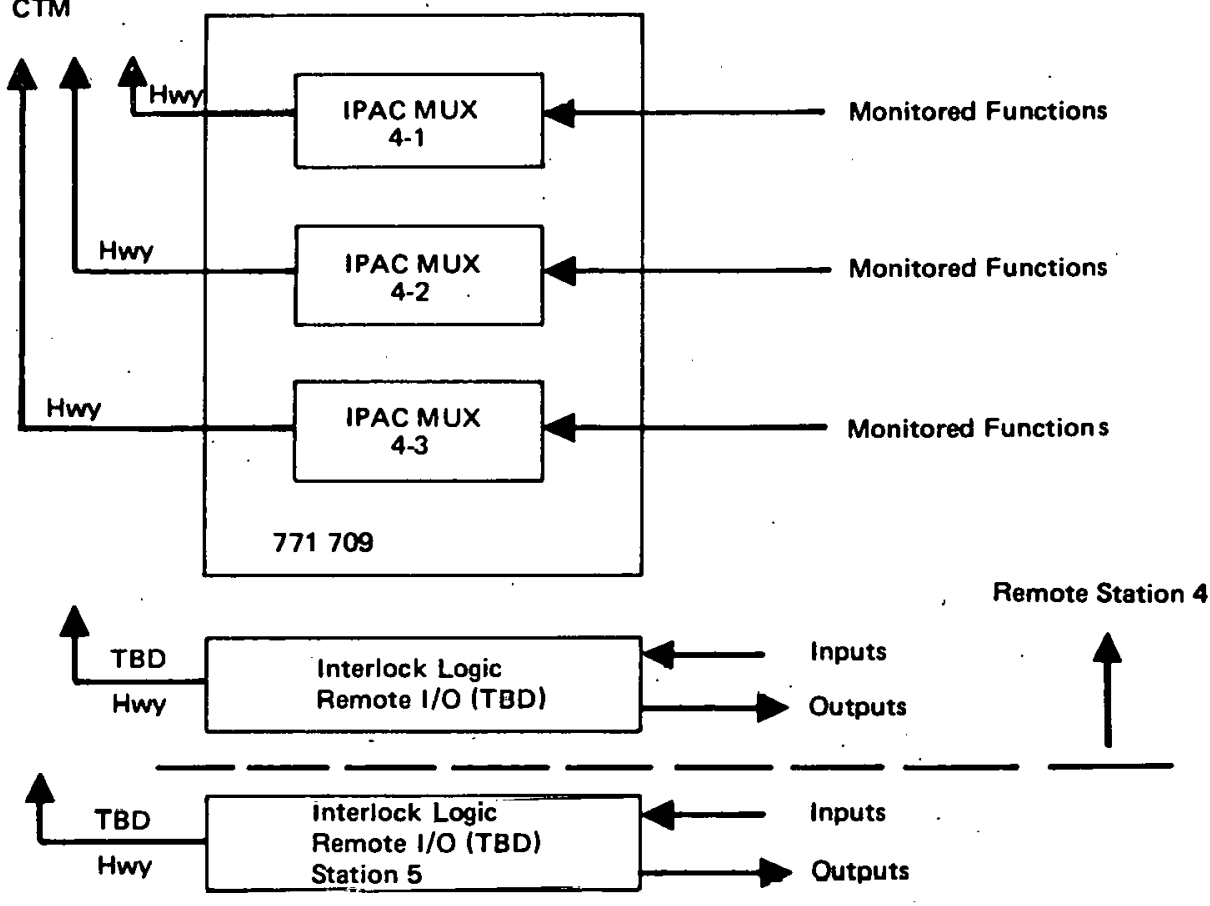

Figure 3-25. SDPC Remote Station -4 , and 5 
Hardware

The red line units for the Receiver Subsystem and Thermal Storage Subsystem to be tested will be supplied by Rocketdyne. Diagnostic test support software furnished by Rocketdyne will be used to demonstrate the hardware readiness of the RLU.

\section{Software}

The red line unit software to be tested will be supplied by Rocketdyne. The red line units will be configured by loading this software.

\subsubsection{Master Control Test Set}

The Master Control Test Set will be an assembly of test equipment which will provide input signals to the SDPC and DARMS, and provide measurement and calibration references. The equipment planned consists of:
A. Transfer function analyzer
B. Oscilloscope
C. Precision DC power supplies
D. Simulated end instruments
1. Strain gage bridge
2. RTT'
3. Signal and calibration voltages
4. Current sources
E. Electronic multimeter
F. Control switching panel

\subsubsection{Major Interfares}

Figure 3-9 illustrates the major interfaces in the Pilot Plant controls test configuration. The external interfaces are those that are the responsibility of the supplier of the equipment or is between equipment supplied by subcontractors or associate contractors.

\section{OCS Interfaces}

The major OCS interfaces are:
A. OCS to HAC A
B. OCS to $H A C B$
C. OCS to Real Time Clock
D. OCS to SDPC 

E. OCS to DAS
F. OCS to BCS
G. OCS to OCS color CRT terminals

Figure 3-26 is a block diagram of the OCS major interfaces and the data rate capability between them. The OCS software related to the HAC will be demonstrated by communicating with limited simulation software resident in the DAS computer. The HAC computers and software will not be available to the SIL.

\section{Major DAS Interfaces}

The major DAS interfaces are:
A. DAS to SHIMMS
B. DAS to HAC A
C. DAS to HAC B
D. DAS to Real Time. Clock
E. DAS to SDPC
F. DAS to DARMS
G. DAS to MDAC PDP-10
H. DAS to DAS color CRT terminals

Figure 3-27 is a block diagram of the DAS major interfaces and the data rate capability between them. The DAS software related to the HAC will be demonstrated by communicating with 1 imited special first software resident in the OCS computer. The HAC computers and software will not be available to the SIL.

\section{SDPC to Receiver and Thermal Storage Electronics}

The SDPC to receiver and thermal storage electronics includes an interface with input process variable signal conditioning, process monitor signal r.nnditioning, unconditioned monitor inputs, and high level discrete talkback and interlock signals (SCU). The SDPC also interfaces with the Red Line Units in both a functional operating and monitoring mode. 


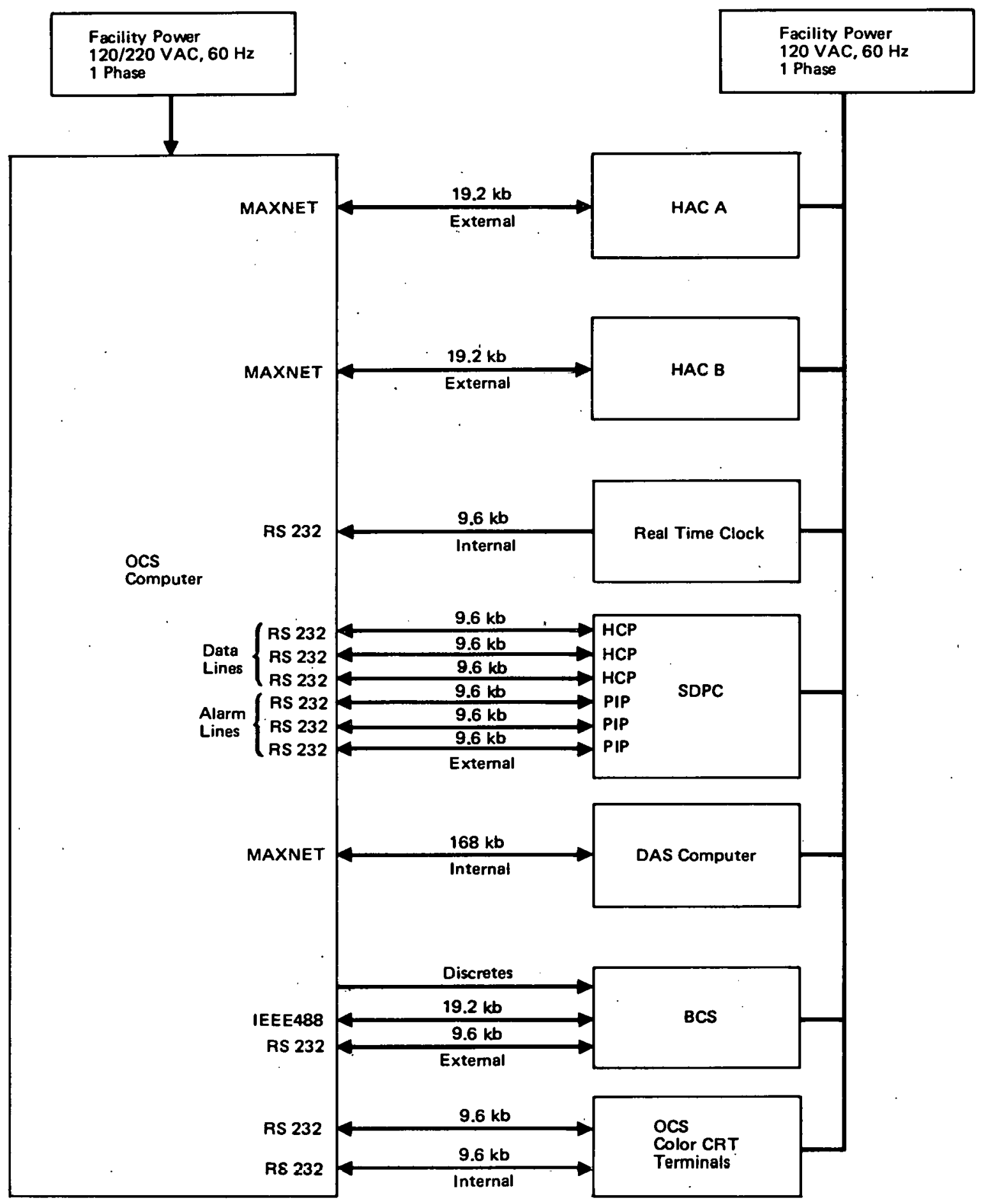

Figure 3-26. OCs Computer Major Interfaces 


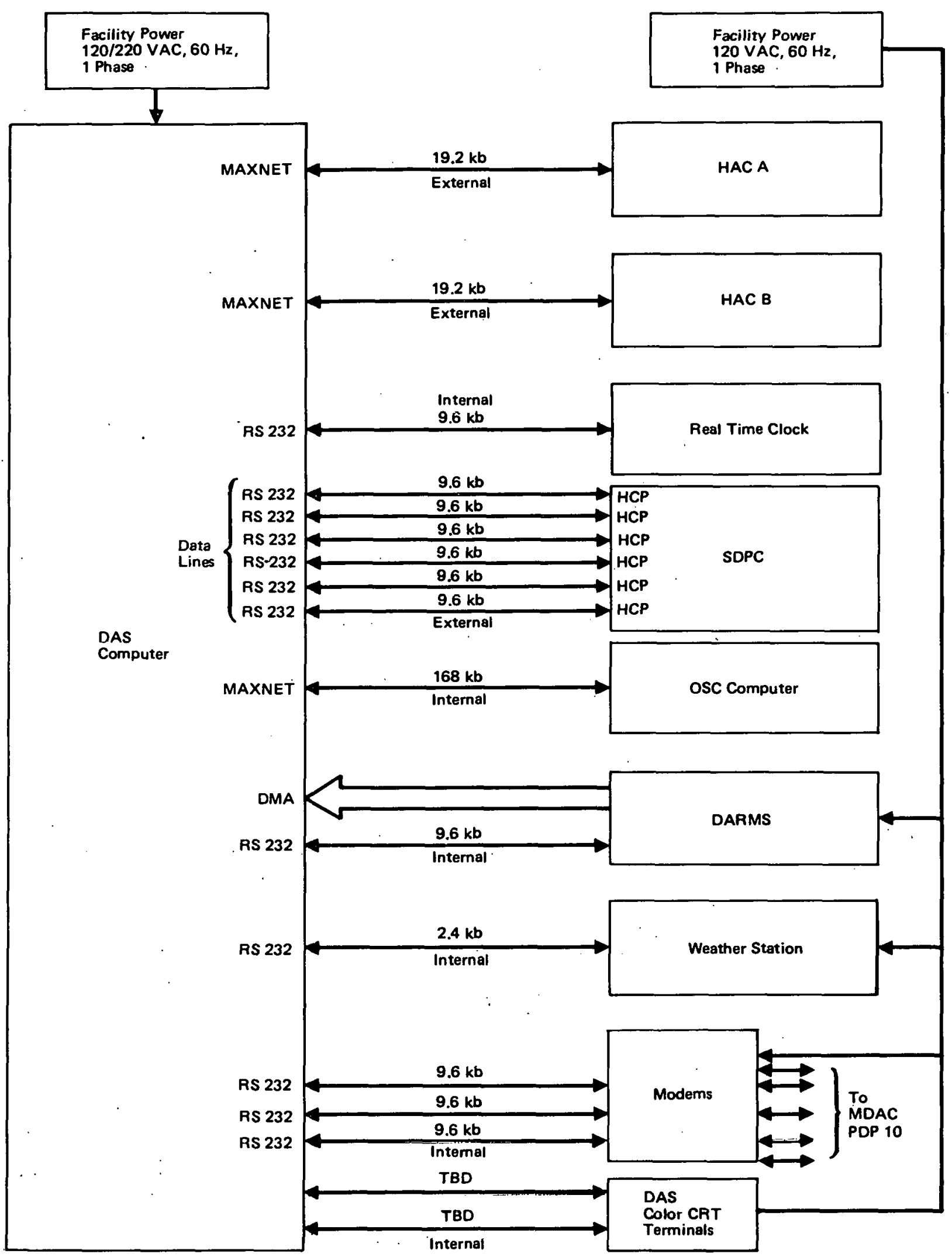

Figure 3-27. DAS Computer Major Interfaces 


\subsubsection{Minimum SIL Test Configuration}

The minimum SIL test configuration will include the minimum hardware necessary to support application software development and field changes and demonstrate their capability within limits.

\subsubsection{Hardware}

The major elements of the mini SIL hardware, illustrated in Figure 1-3, are a MODCOMP 7861 computer, Beckman operator programmable control unit (OPCU) terminal, and Beckman multivariable control unit. MODCOMP software diagnostics will be utilized to demonstrate the MODCOMP computer and peripheral readiness. Beckman "fill-in-the-blanks" firmware will be used to configure and operate the Beckman elements.

\subsubsection{Software}

The following software will be developed for the OCS computer using the computer resources of the mini SIL:

A. OCS Tool Software

1. Logging

2. Supervisory control routines

3. Data base

4. Man-machine interface

B. OCS Applications Software

1. Supervisory control

2. Coordinated control

3. Clear day control

\subsection{INTEGRATION TESTING}

\subsubsection{Maxi SIL}

The hardware interfaces between subsystems will be demonstrated to be functionally operable to assure readiness to support system integration. After hardware readiness has been established, tests will be performed that functionally demonstrate a hardware and software linkage capability between each interface. Performance under conditions such as an electrical noise environment, induced errors, maximum throughputs, and recovery conditions (power failure) will be evaluated (see Figure 3-28). 


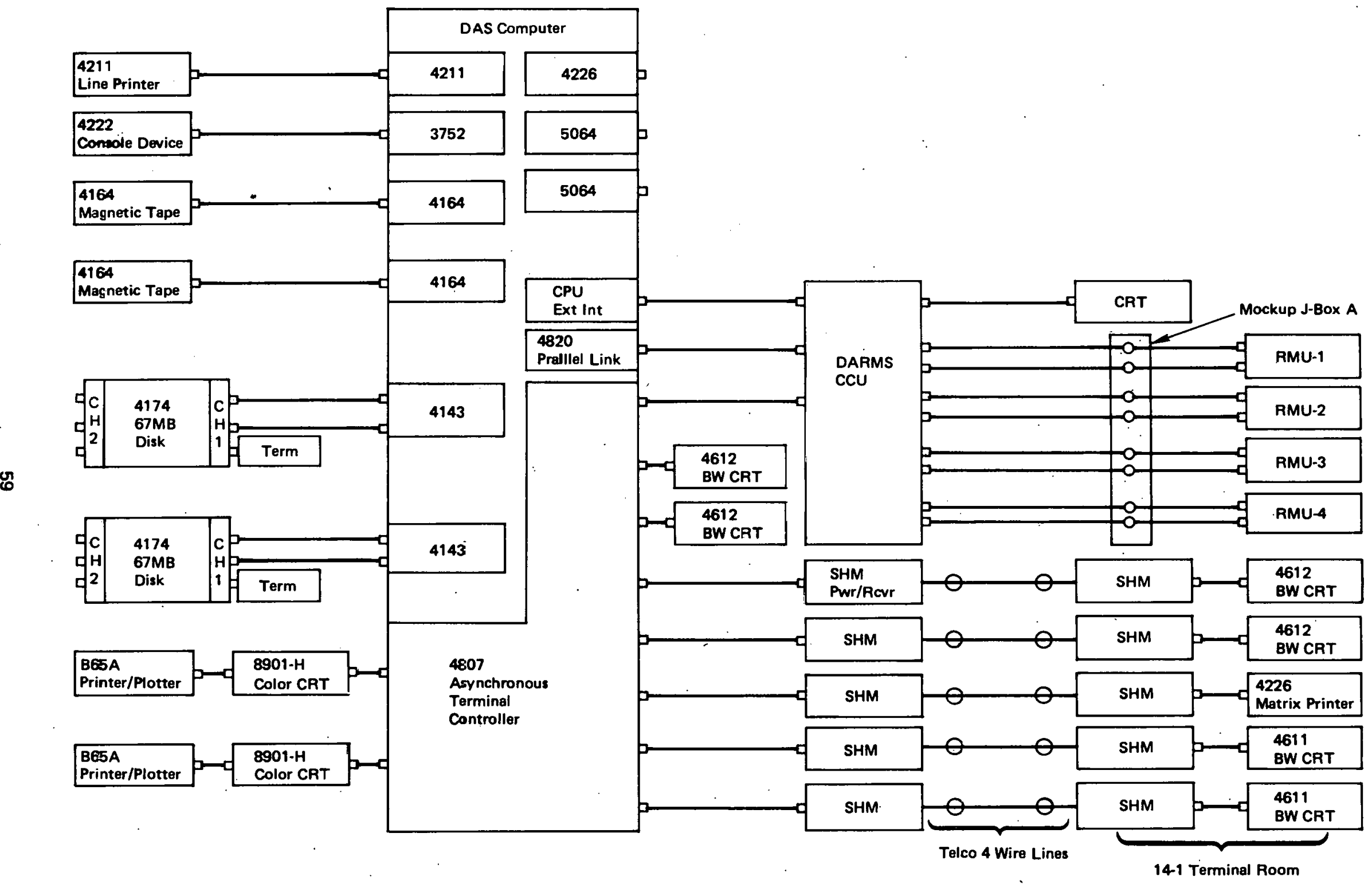

Figure 3-28. Stage I Equipment Configuration 


\subsubsection{Hardware}

Representative tests will be conducted, insofar as possible in the system integration laboratory, to demonstrate:

A. SDPC controls including subsystem signal conditioning and red line unit operation using manual operating procedures

B. DAS system including MCS test set simulated inputs, DAS application software, and all major interfaces

C. OCS system including MCS test set simulated inputs, OCS tool software, and all major interfaces

D. Master Control Test Set

\subsubsection{Test Control Drawing}

The SIL activities will be planned, controlled, and documented by the use of a Test Control Drawing (TCD). This drawing will identify and define the requirements for the system checks to be performed during the various stages of test. It will specify test configurations both physical and electrical and identify any special requirements for running the test. It will provide the means to document results of individual tests and provide a means for overall evaluation of the system operation.

\subsubsection{Mini SIL}

The interfaces between all elements of the mini SIL equipment will be demonstrated to be functionally operable. These interfaces will not be evaluated to the restrictive conditions previously tested in maxi SIL, since the interfaces of the mini SIL equipment is similar.

Representative tests will be conducted within the limits of the mini SIL equipment to demonstrate the OCS tool software completion, OCS application software, and "field fix" software. 
Section 4

BCS SIL OPERATION

The BCS System will be assembled, configured into test modes, and checked out for proper hardware and software operation to the extent described in this section. The equivalent of SIL testing for the BCS hardware and software will be performed in parallel with, but independent of SIL, using a surrogate MODCOMP CLASSIC computer in place of OCS computer linked to the basic complement of BCS equipment. The major portion of the BCS interface and integration testing (Phase I) will be performed at the Solar Energy Test Facility (SETF) site which is separate from the SIL but also located at the MDAC-HB facility (Figure 4-1). The SETF site provides the capability to test the BCS using redirected light beam images on target surfaces generated by heliostats tracking the sun.

$B C S$ integration functions also require magnetic tape operations as well as operations with MAXNET software installed in the OCS computer. These integration functions will be performed in Stage VI of SIL, when the BCS hardware and software will be removed from the surrogate computer and installed with the OCS computer in the SIL facility.

\subsection{BCS TEST OBJECTIVE}

The tests conducted on the BCS system will verify the operational integrity of the hardware and demonstrate the proper software operation in interfacing with the OCS and DAS computers and peripheral equipment. The BCS hardware to be tested is illustrated in Figure 4-2. The COHU video camera system black/white CRT keyboard/hardcopier terminal with built in hard copler diagrammed in Figures 4-3 and 4-4 respectively along with the MODCOMP computer and peripherals, video digitizer, and Modular Data Acquisition and Control System III (MODACS III) makeup the system hardware.

The BCS will test heliostat reflected beam quality. MODCOMP software, processed dala from beam quality tests, and diagnostic software will be used to 

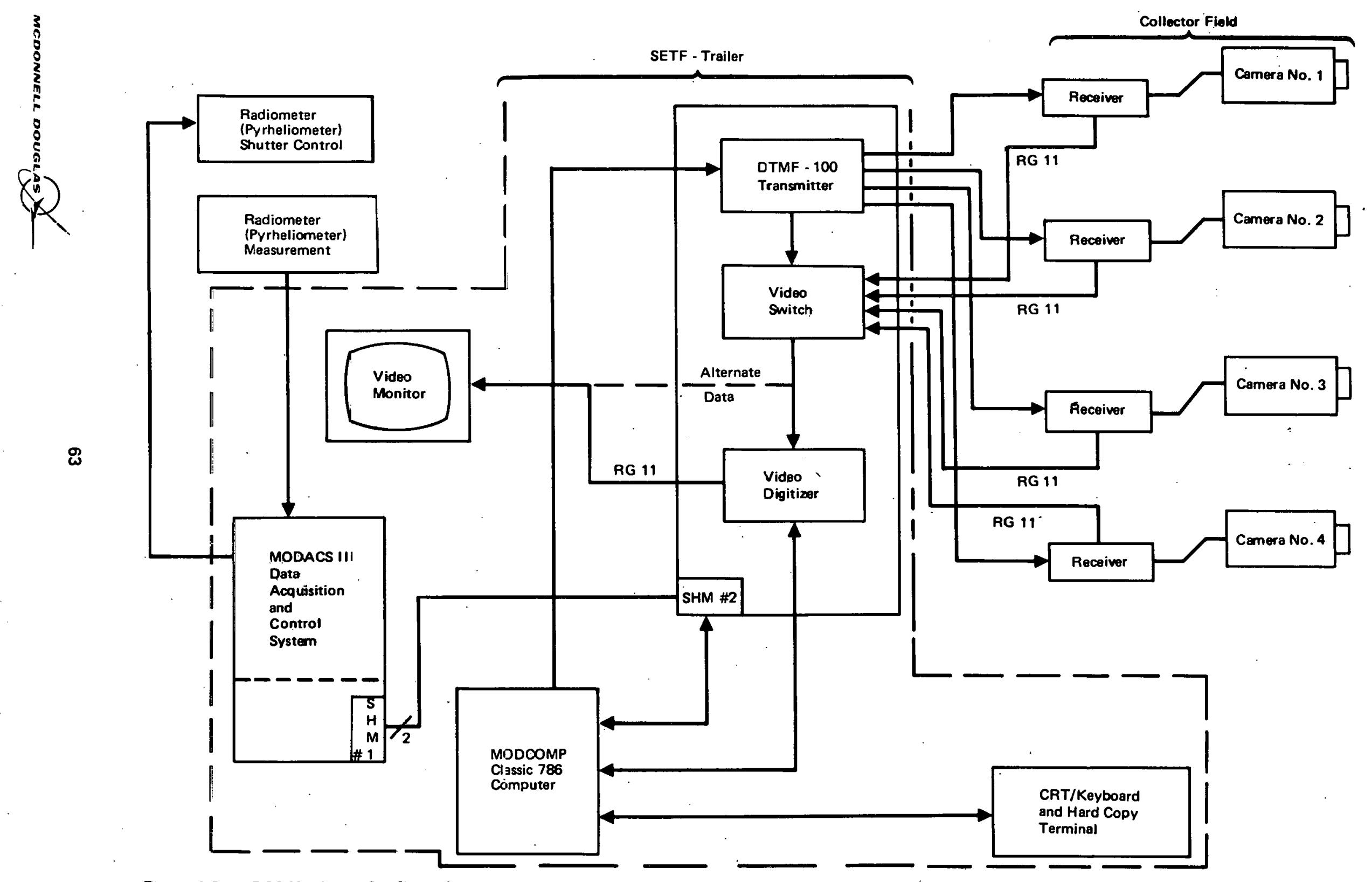

Figure 4-2. - BCS Hardware Configuration 


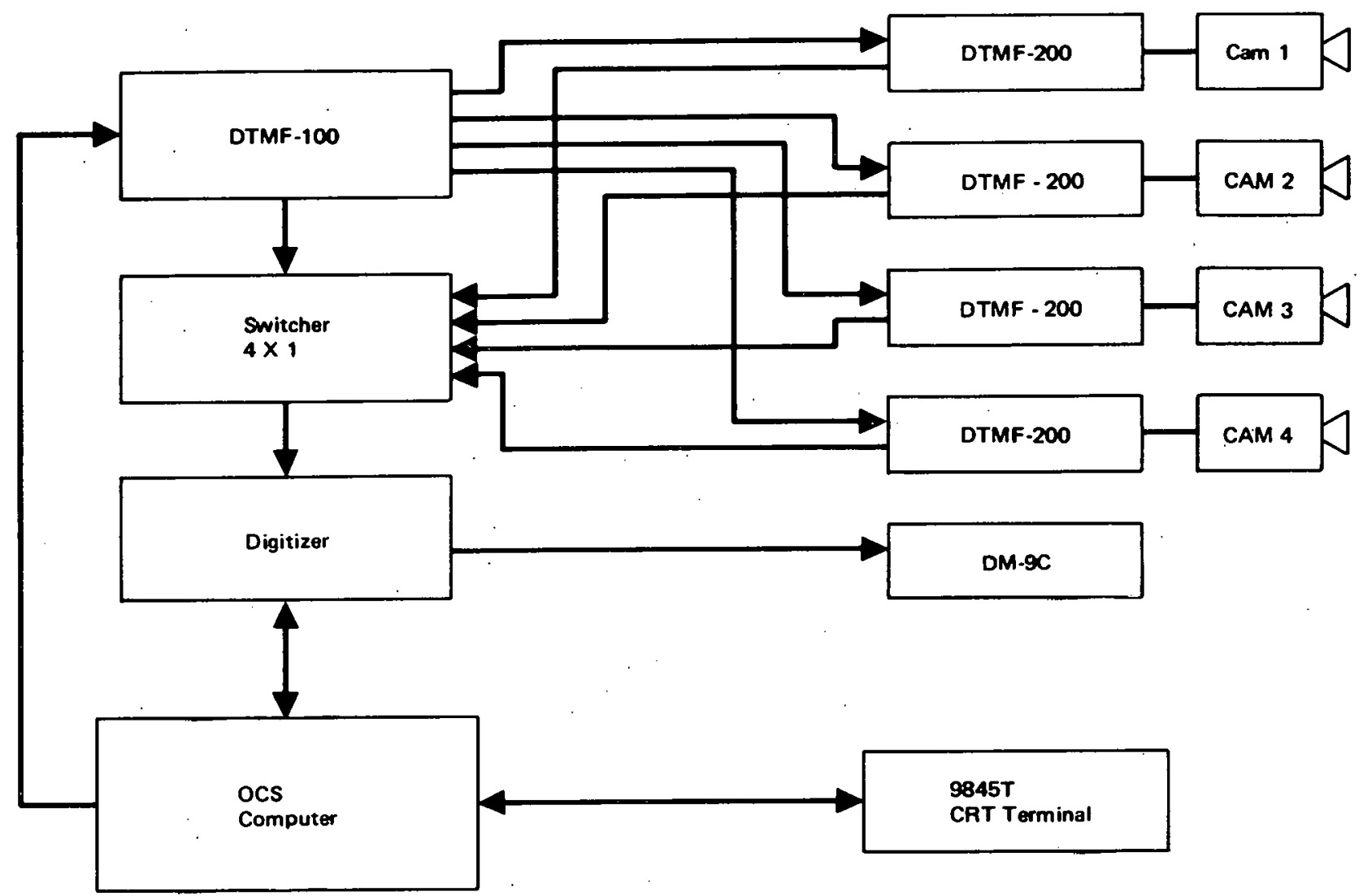

Figure 4-3. Video Camera Configuration

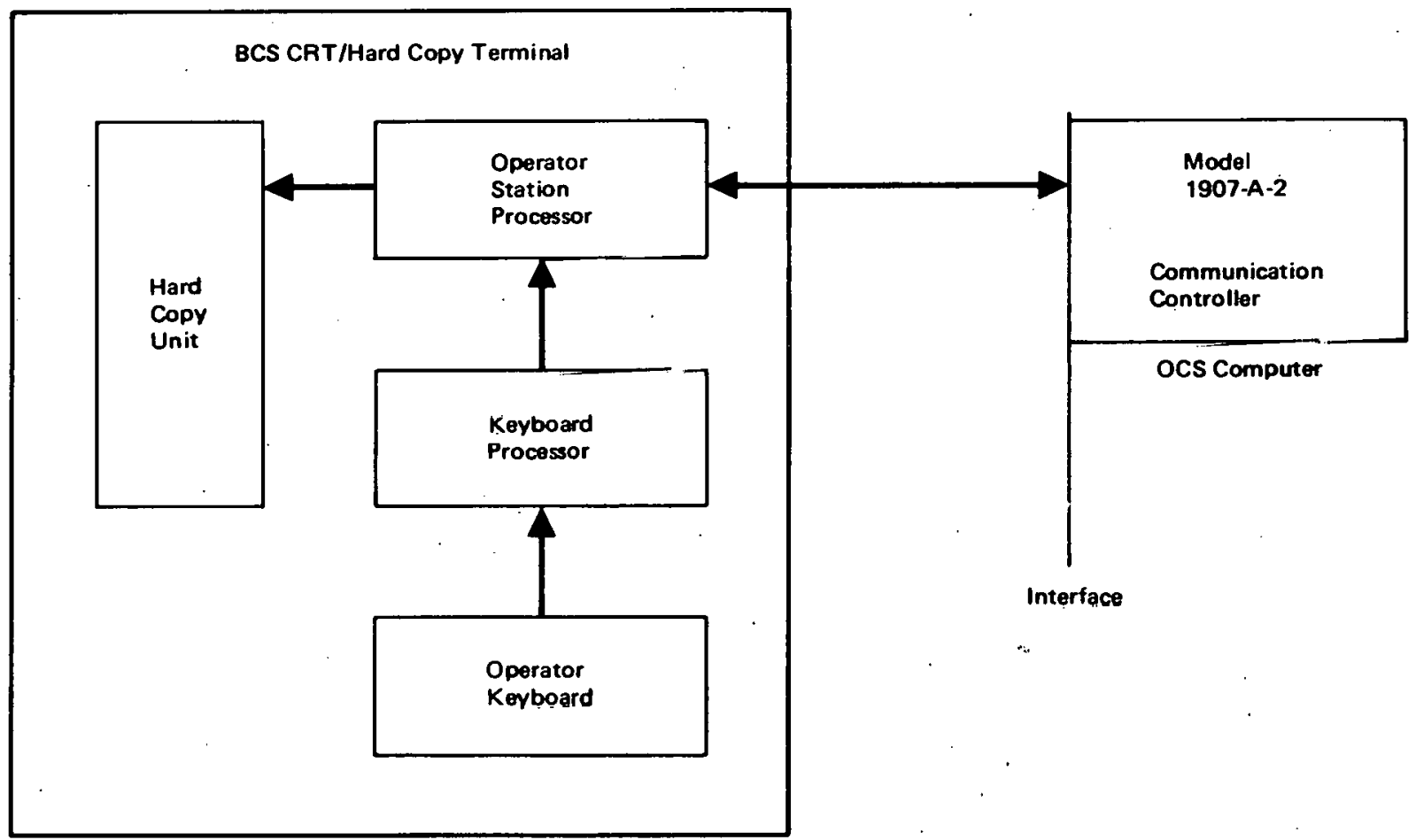

Figure 4-4. BCS BN CRT/Hard Copier Terminal Block Diagram 
demonstrate MODCOMP hardware readiness. Applications and test support software will be used to verify the internal interfaces between the MODCOMP computer, the CRT/hard copier terminal and the video camera system.

\subsection{BCS TEST FACILITY}

The BCS and external interface operational functions will be integrated and functionaliy demonstrated at the SETF as a part of SIL activities. The BCS will be checked out in two phases. Phase I will be performed outside of the main SIL area at the SETF site and will interface with a surrogate OCS computer. Phase II will utilize SIL to checkout the software and hardware interfaces with the deliverable ocs computer.

\subsubsection{BCS Phase I Activities}

The following activities will be performed during Phase I:
A. Prepare BCS test facility
B. Define BCS Phase I equipment layouts
C. Define BCS special cable requirements
D. Fabricate Phase I cables
E. Define special BCS test equipment
F. Assemble special BCS test equipment
G. Install BCS equipment physically per Figure 4-1
H. Connect test configuration per Figures 4-5, 4-6, and 4-7
I. Perform hardware functional testing
J. Perform hardware integration testing
K. Perform software integration and verification testing

\subsubsection{BCS Test Facility Preparation}
A. Define and install electrical power for BCS equipment
B. Define and fabricate temporary camera pedestals

\subsubsection{BCS Phase I Equipment Layouts}
A. Define physical locations for all equipment
B. Define electrical power cable locations
C. Define signal cable routing 
Trailer SETF

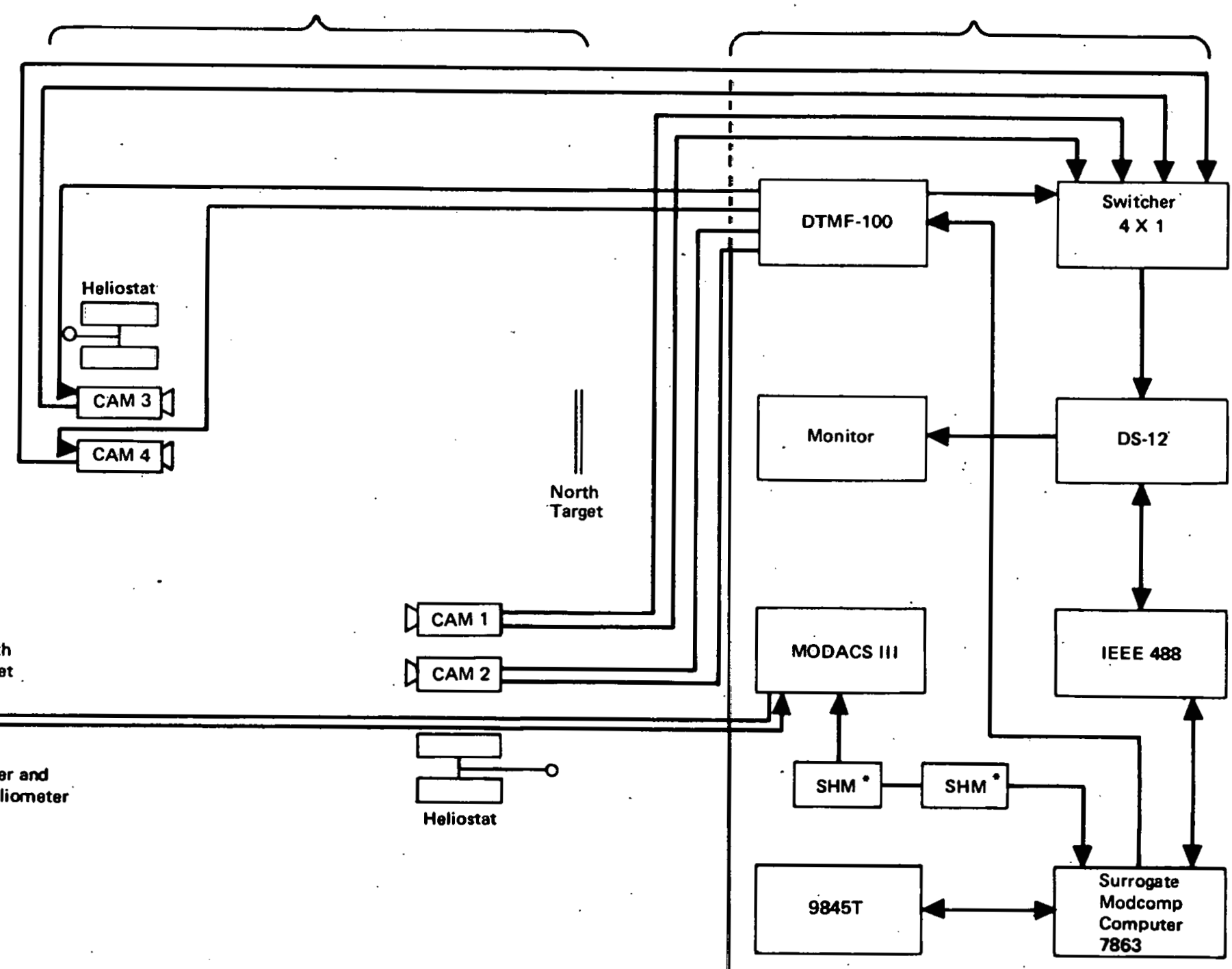

"SHM-Short Haul Modem 


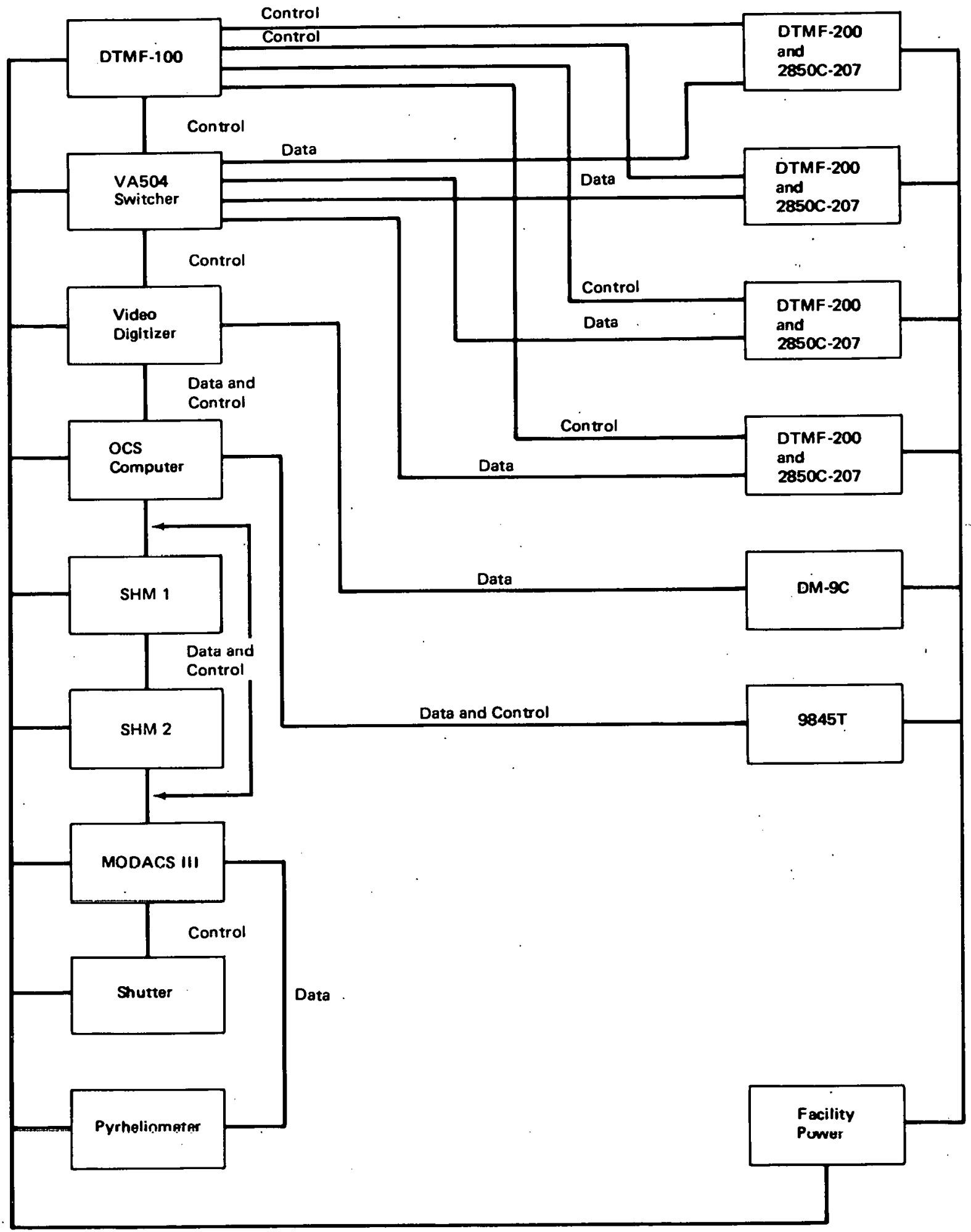

Figure 4-6. BCS Internal/External Interface Block Diagram 


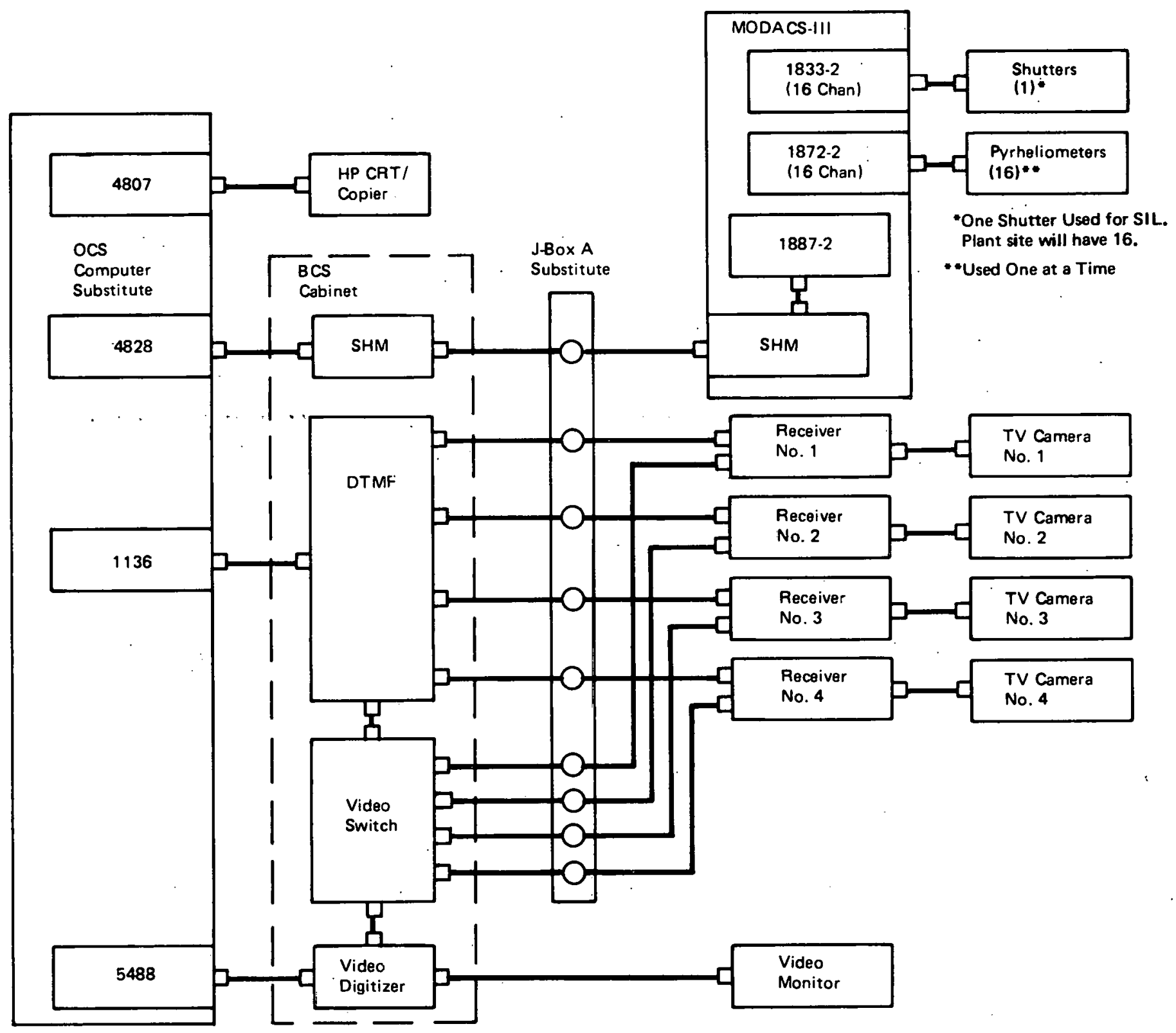

Figure 47. Solar SIL - BCS Equipment Configuration 


\subsubsection{BCS Special Cable Requirements}

A. Define and provide a fabrications drawing for a temporary power cable

B. Define and provide a fabrication drawing for temporary signal cables

\subsubsection{Phase I Cable Fabrication}
A. Fabricate special power cables
B. Fabricate special signal cables

\subsubsection{Special BCS Test Equipment}

Obtain and place into readiness the following special test equipment:
A. Waveform Monitor - Textronic RM529 or equivalent
B. Camera Test JIG - COHU Model CTJ-2
C. Bar-Dot Generator - COHU Mode1 2380-004
D. Volt/Ohmeter - Fluke Model 8500A or equivalent
E. Camera Lens Control Unit (CLCU) - COHU Model
F. Active Cavity Radiometer -

\subsubsection{BCS Hardware Functional Testing}

The following BCS hardware functional tests will be performed with the equipment installed in the SETF.

\section{Camera Tests}

Camera checkout will be made by performing an initial continuity check on all interconnecting cables. The system will then be hooked up as shown in Figure 4-6 and the camera lens control unit (CLCU) will be utilized to adjust and calibrate the zoom, focus and iris of each camera. (See Figure 4-8)

\section{Shutter Test}

Verification of shutter operation will be made manually by activating the on/off switch on the front panel and observing that the shutter responds correctly to the open and close commands. 


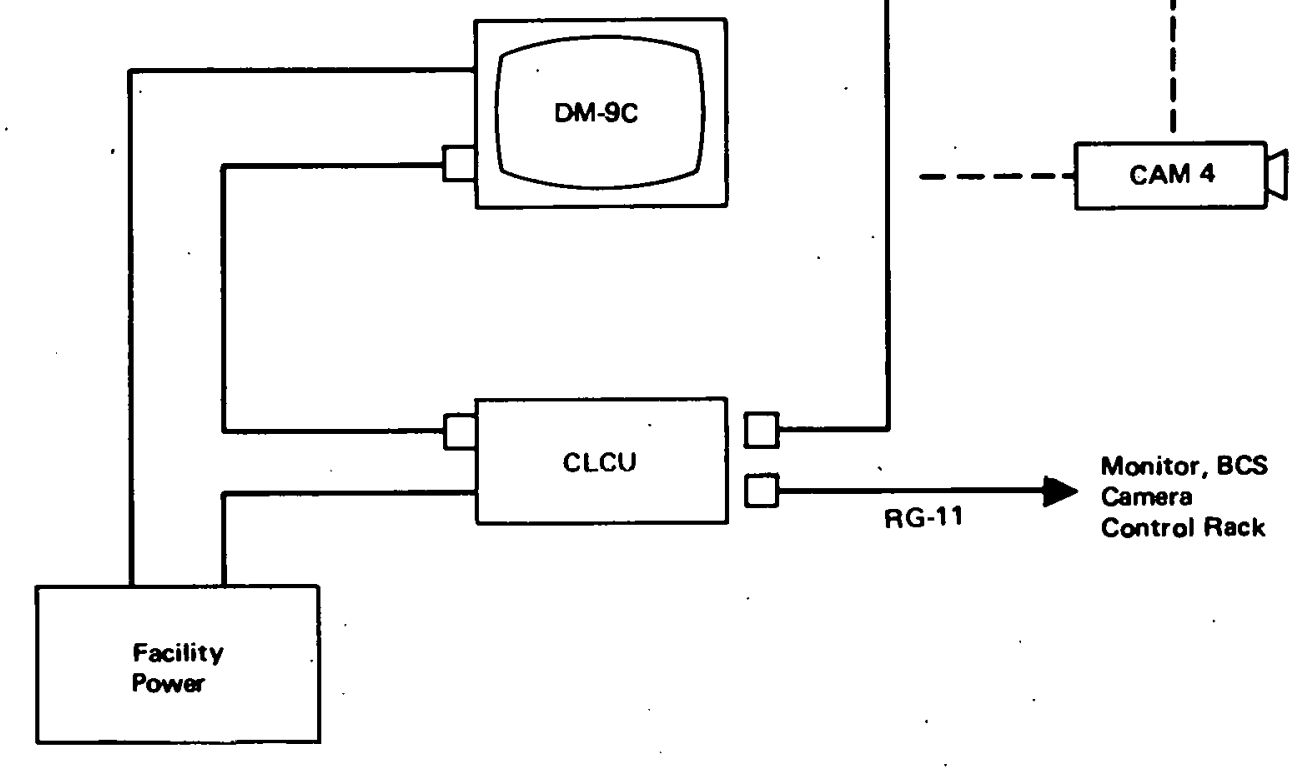

Figure 4-8. CLCU Setup Configuration

\section{Pyrheliometer Tests}

Each of the 16 BCS pyrheliometers will be tested to determine first, if they are operational and second, if they are calibrated properly.

Hardware Integration Testing

Hardware integration testing will be performed with all hardware components connected to the surrogate OCS computer. Computer test software will verify hardware interfaces. The equipment will be connected as shown in Figure 4-2. The operator will operate the BCS as a system through the BCS CRT/keyboard terminal. Tests will be conducted to check auto/manual control, iris control camera switching, data acquisition, data display and hardcopying functions.

\subsubsection{Software Integration and Verification Testing}

Software integration and verification tests will demonstrate the following:

A. That individual modules perform according to the BCS requirements.

B. That BCS software modules can communicate with each other and perform in a coordinated manner. 
C. That BCS software can exercise the major hardware interfaces of BCS.

D. The BCS software can demonstrate the ability to command certain BCS hardware that includes:

1. The camera

- For camera switching to digitizer

- Iris control

2. The shutters (via MODACS III)

3. The pyrheliometers (via MODACS III)

4. The BCS display/hardcopy terminal

E. Verify the BCS/HAC interface operation to the extent possible using simulated or externally applied inputs and verify the ability of the BCS software to process all input messages and generate all output messages as specified in the CS to MCS interface specification RADL 2-30-1.

F. Demonstrate the operation of the BCS software in the OCS computer under supervision of the MAXNET operating system and with other non related tasks sharing the computer resources concurrently.

In accordance with the above, the following subparagraphs specify the software verification procedure for the major software functions of BCS to be demonstrated during Phase I testing.

Figure 4-9 depicts the BCS top level functional flow and illustrates the relationships between the major software components of BCS. Figure 4-10 illustrates the primary test configuration to be verified during Phase I testing.

\section{BCS Initialization Function}

The BCS initialization function will be activated at the BCS terminal and its operation will be verified by printout of the initialized data base, and real time files and observing and verifying the prespecified man-machine dialogue at the terminal.

\section{HAC Input Executive}

The HAC Input Executive function will be verified by supplying all BCS messages from the collector system (CS) to the OCS as specified in RADL 2-30-1 over a simulated RS-232 link defined under the MAXNET operating system. 'This 


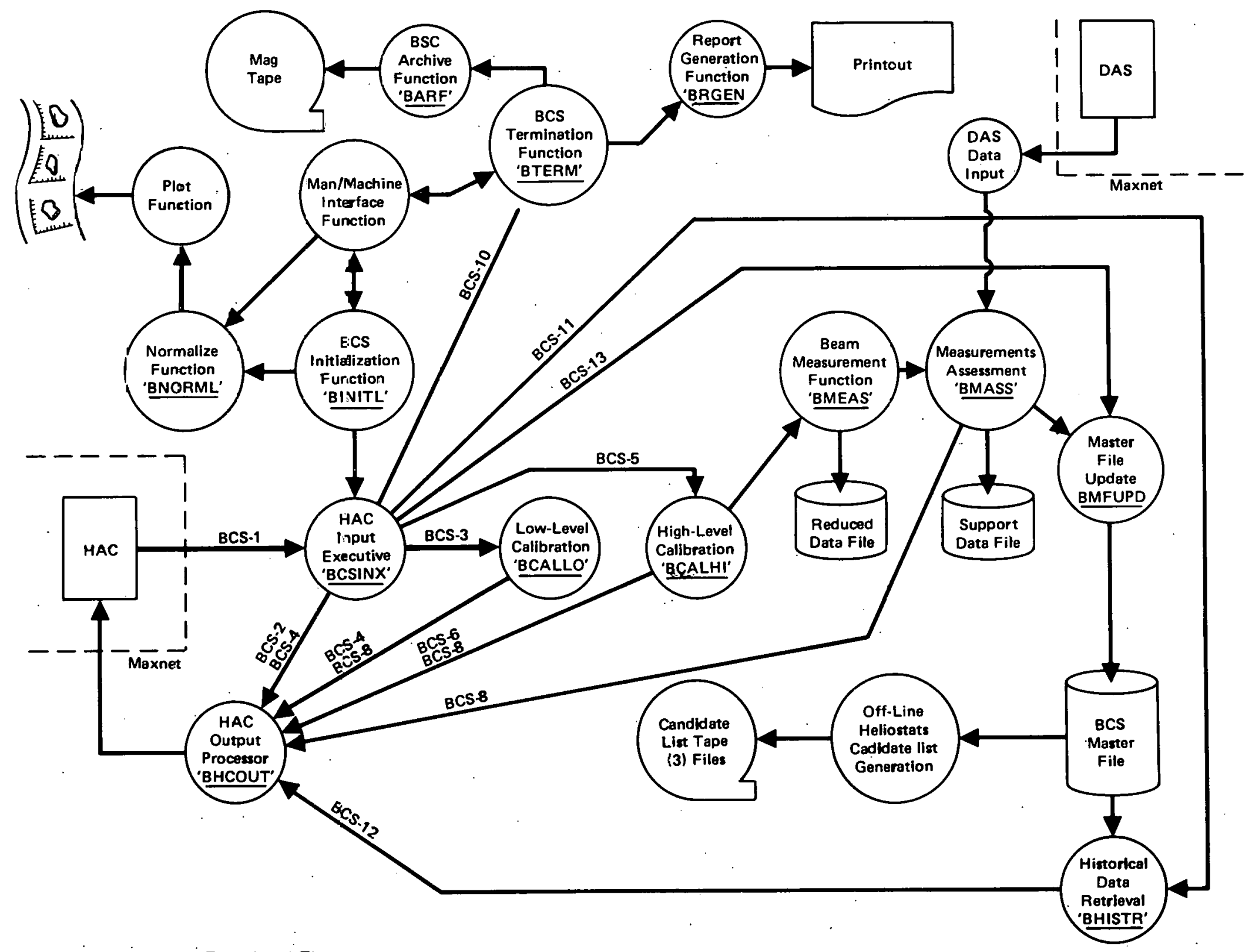

. Figure 4-9. BCS Top Level Functional Flow 


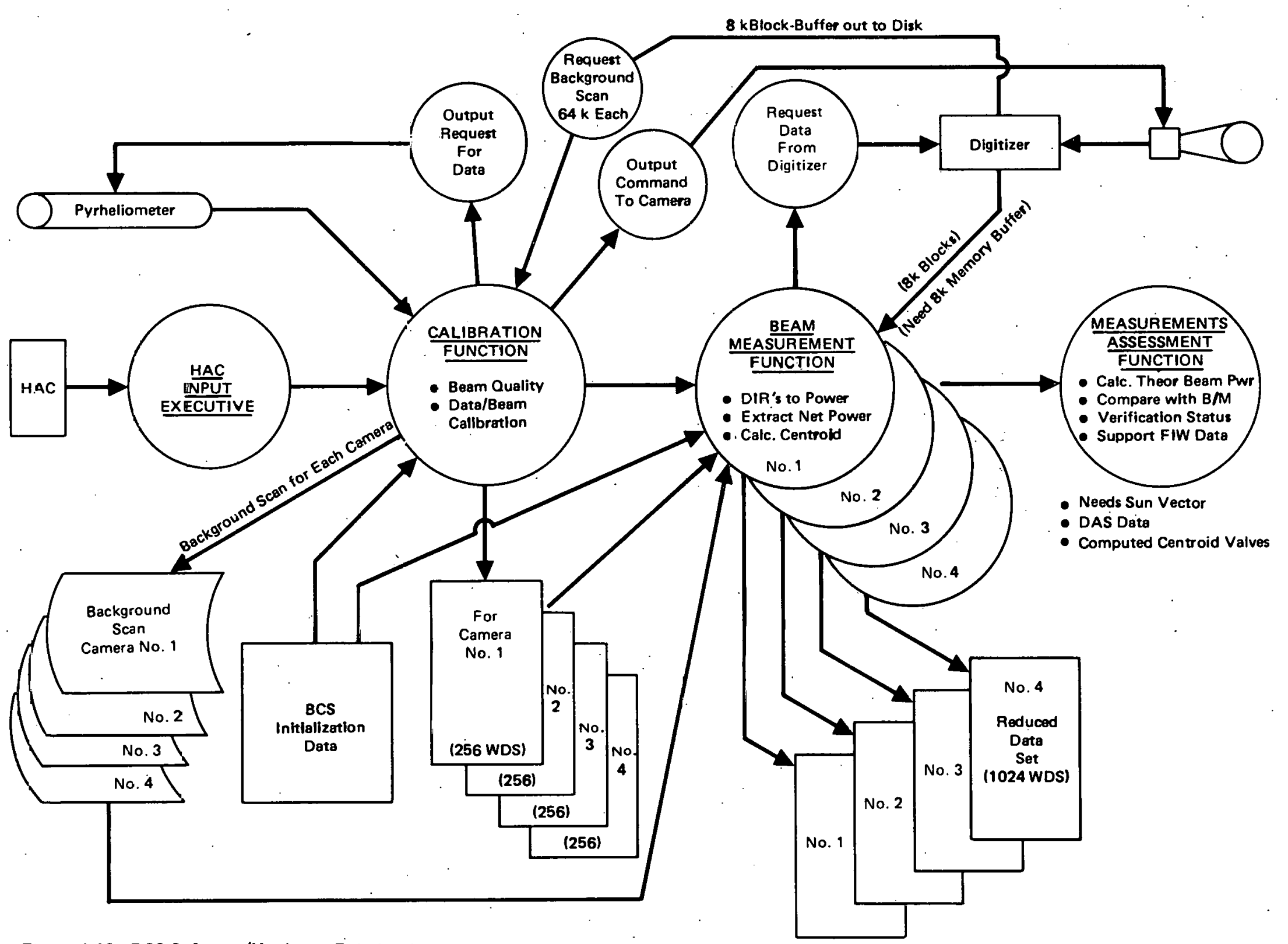

Figure 4-10. BCS Software/Hardware Relationships 
function will be the primary driver for other BCS functions which will be demonstrated MODCOMP supplied software.

HAC Output Processor

The HAC output processor function will be verified by activating test sequences which result in the request for generation of all BCS messages acceptable by the CS from the OCS as specified in RADL 2-30-1. Constructed messages will be directed to the line printer for manual verification of structure and content.

\section{Low-Level Calibration Function}

MODCOMP supplied software will be exercised to demonstrate the low-level calibration function. The capability of the BCS software to communicate with the pyrheliometers and the shutters through the MODACS III processor via MAXNET shall be verified. In addition, the capability to request and process data from the video digitizer via the IEEE-488 interface will be exercised. This function will also provide verification that the BCS software can determine if a beam is off the target, that it can command the switching of cameras as a function of target selected, obtain Digital Image Radiometer (DIR) values and pyrheliometer measurements and obtain and store background scan data for subsequent use.

\section{High-Level Calibration Function}

The high-level calibration function will also exercise and demonstrate the capability of BCS software to command the cameras, receive data from the digitizer via the IEEE-488 interface and command the shutters and the pyrheliometers through the MODACS III processor via MAXNET. This shall be accomplished using SFDI developed software. This function will provide verification that the BCS software performs the following functions:

A. Determine if the beam is on target.

B. Verify the data range and exposure from the camera.

C. Command the cameras for iris adjustment, switching, and image grab.

D. Perform high-level calibration and construct calibration tables. 
Beam Measurement Function

The beam measurement function also communicates with the camera and the digitizer via the IEEE-488 interface. Therefore, this software module will also serve to verify the interface between the camera, the digitizer and MODCOMP computer. This shall be accomplished using SFDI developed software. The following BCS functions will be verified by execution of this BCS software component:

A. The capability to command the switching of cameras.

B. The capability to command the camera to take an image grab.

C. Processing of data received from the digitizer.

D. Performance of power calculations.

E. Calculations of beam centroid.

F. Generation of reduced data file information.

\section{Measurements Assessment Function}

The measurements assessment function will be activated by the Beam Measurement function and will demonstrate the capability of the BCS software to calculate the theoretical beam power and to update latest calculated measurements information on the support data file. Subsequent printout of the updated data files will be used as a method of verification.

\section{Master File Update}

The master file update function will demonstrate the capability of the BCS software to update selected parameters on the BCS master file. This function will be activated by a special test driver as well as by the Measurements Assessment function; verification will be accomplished by printout of updated data files.

\section{Normalize Function}

The BCS normalize function will perform calculations of data contained in the reduced data file and will prepare data for the presentation of a contour plot which provides a means of assessment as to the calibration of a particular heliostat. SFDI developed software shall be exericsed to verify this function.

\section{Plot Function}

The BCS man-machine interface software will be utilized to verify the capability of the BCS plot function software to generate selected contour 
plots on the BCS terminal. This function will also demonstrate the capability to produce hardcopy output of displayed plots at the BCS terminal upon operator request. Verification shall be accomplished by observation of plotted information on the CRT and comparison of the hardcopy output to the display.

\section{Historical Data Retrieval}

A special test driver shall be utilized to exercise and verify the historical data retrieval function. The capability of the BCS software to extract information from the $B C S$ master file, required for bias calculations in the $H A C$, and set the data up for transmission to the HAC will be verified by directing $H A C$ output messages to the printer and verifying that the proper information was retrieved and properly formatted.

\section{BCS Termination Function}

The BCS termination function will be activated via special test driver to demonstrate the BCS functions can be orderly terminated upon receipt of a unique message from the HAC. In addition, a prespecified man-machine dialogue which presents various BCS termination options will be verified by observations at the BCS terminal.

\section{Man-Machine Interface Function}

The man-machine interface function of BCS. will be demonstrated using the BCS terminal and invoking various operational sequences mechanized within the initialization, termination, plot and report generation functions of the BCS software, and observing the display of special prompts and fill in the blank formats. Verification that manual inputs have been accepted by the BCS software will be done with special test software to read and subsequently print out information of interest.

\section{Report Generation Function}

The report generation function will demonstrate the capability of the BCS software to generate reports on a line printer device in a prespecified format. This function shall be activated via the BCS man-machine interface and verified by examination of the generated printer output. 


\subsubsection{BCS Phase II Activities}

The following activities will be performed during Phase II:

A. Layout BCS equipment Phase II configuration in test facility area in SIL.

1. Allocate physical location of BCS hardware

2. Establish power receptacle locations for BCS hardware

3. Layout signal cable routine from equipment bay to equipment

bay

B. Define BCS special. Phase II cable requirements

1. Define and provide fabrication drawings for Phase II power cables

2. Define and provide fabrication drawings for Phase II signal cables

C. Fabricate special Phase II cables

1. Fabricate special power cables

2. Fabricate special control

D. Define special BCS test equipment from equipment listed in Section 4.2.1.5

E. Install BCS equipment in the Phase II test configuration

1. Dismantle Phase I test set up

2. Relocate equipment per Figure 3-2.

F. Connect test configuration per Figure 4-11

G. Perform hardware functional testing

H. Perform hardware integration testing

I. Perform software integration and verification testing

\subsubsection{Hardware Functional Testing}

Hardware functional testing will be performed in accordance with Sections 4.2.1.6.7 then 4.2.1.6.3.

\subsubsection{Hardware Integration Testing}

Hardware integration testing shall be performed in accordance with Section 4.2.1.6.4 where applicable. 


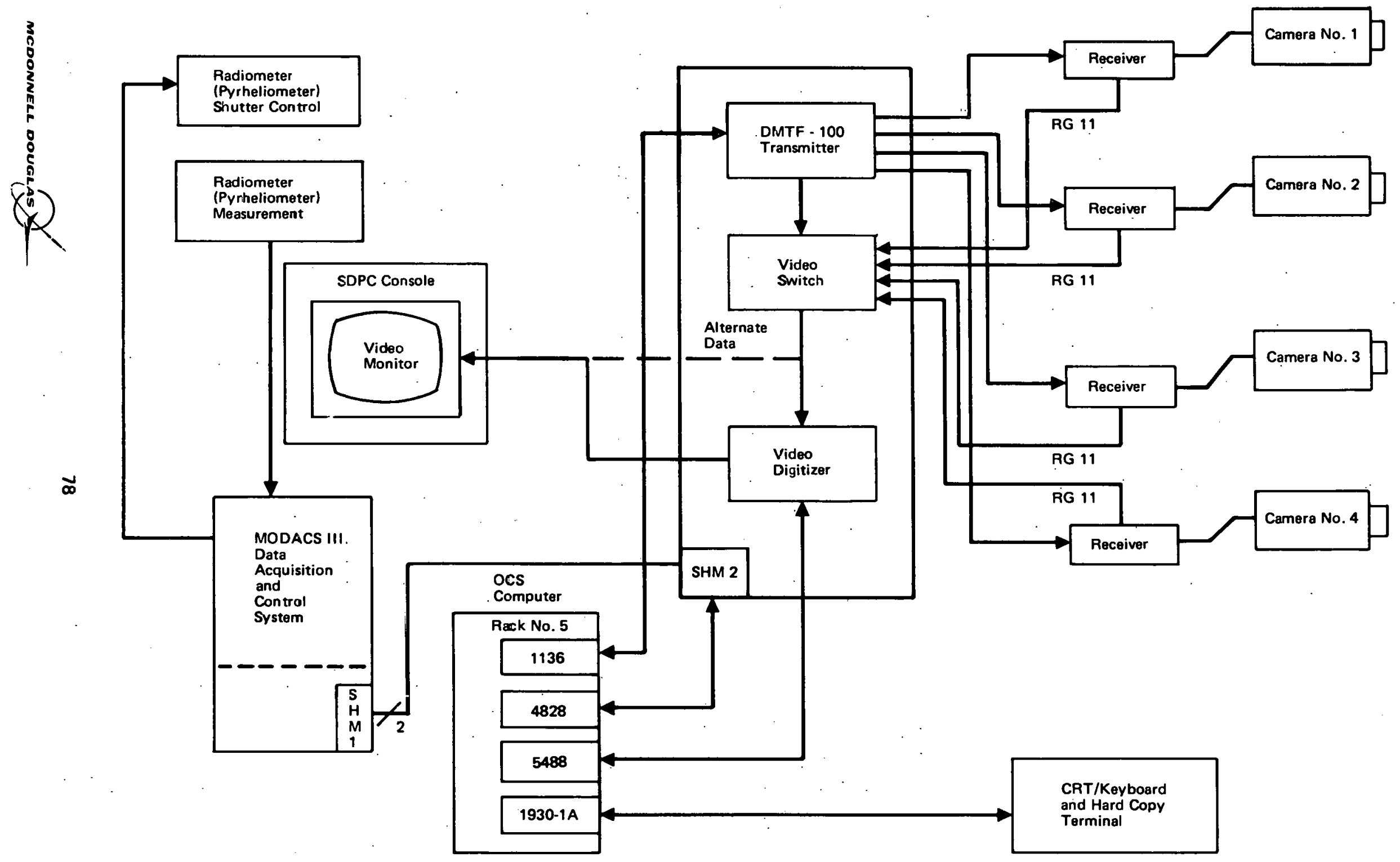

Figure 4-11. BCS Hardware Configuration 


\subsubsection{Software Integration and Verification Testing}

The major complement of the BCS software will have been tested, integrated and verified during Phase I testing. Figure 4-12 indicates with cross hatching those portions of the BCS software deferred to Phase II testing activities. These functions are confined to the software modules interface with the OCS magnetic tape unit as well as the DAS computer via MAXNET. In support of BCS Phase II testing the following functions shall be performed.

A. Install BCS software on OCS computer in SIL

B. Create BCS files as required on the OCS disk

C. Perform software readiness test to determine validity of BCS software configuration

D. Demonstrate the operability of the Off-Line Heliostats Candidate List Generation Function.

E. Demonstrate the BCS archive function

F. Exercise and verify the MAXNET interface between the OCS and DAS computers with respect to acquisition of data for BCS from the DAS computer.

G. Demonstrate operation of the BCS software with other tasks resident in the OCS computer and all tasks executing concurrently.

\section{BCS Software Readiness Testing}

In order to verify the BCS software configuration loaded into the OCS computer a top level software readiness testing activity shall be performed as follows:

A. Exercise the BCS initialization function thereby verifying the man-machine interface and the operability of the BCS terminal.

B. Verify by manual input, expected HAC input commands that will serve to stimulate the operational execution of various BCS functions and list the outputs of those functions.

C. Use reduced data and support data files to verify the operability of the plot function as well as the report generation function on the OCS computer.

\section{Heliostats Off-Line Candidate List Generation}

Since there will not be a magnetic tape unit available in the special BCS test facility, BCS Heliostats Off-Line Candidate List Generation will be 


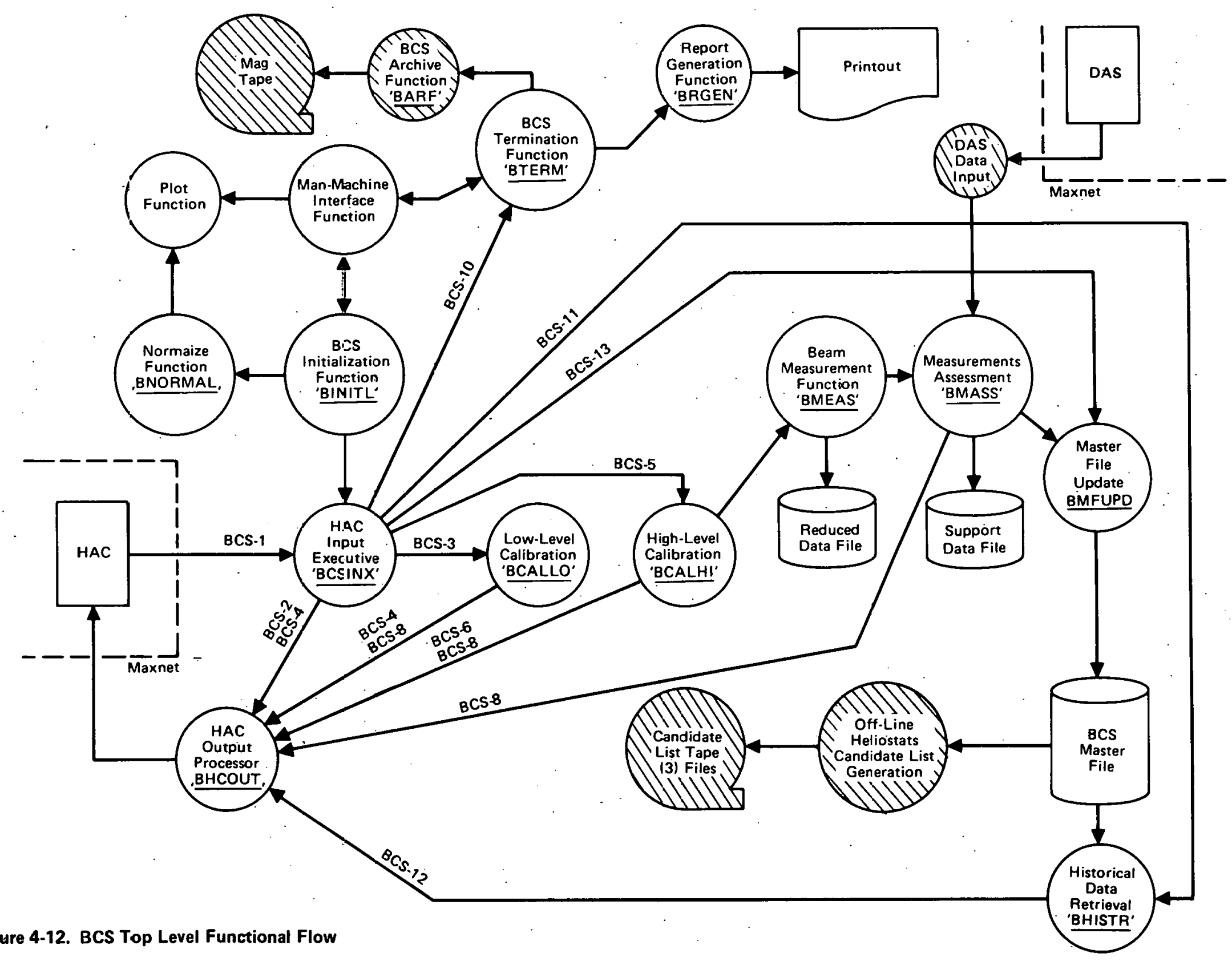


demonstrated in the SIL using the OCS computer and its magnetic tape unit(s). The BCS master file will be used as the primary source of input. Its content $: 1111$ be printed to verify the selection process by this program. The list tape generated will be listed and compared to the BCS master file entries for verification.

\section{BCS Archive Function}

The BCS archive function will be demonstrated in the SIL using the OCS hardware configuration primarily because of the magnetic tape unit availability. A simulated archive tape will be generated using special test software.

The generated tape will be verified by printing the contents thereof and comparing it to a printout of the simulated data which was generated prior to the archive operation. Verification that reinitialization of archived data files has been performed will also be determined through inquiry to the MODCOMP system.

\section{DAS Data Input Function}

The MAXNET interface with the BCS software will be verified for BCS operations in the SIL. Results will be verified by using special test software to generate DAS data. This data will be printed out on the DAS computer printer and printout of data to be transferred to OCS computer, and printed on OCS printer for comparison.

\subsection{BCS SCHEDULE}

The BCS hardware/suftware devclopment and testing schedules are shown in Figures 4-13 and 4-74. 


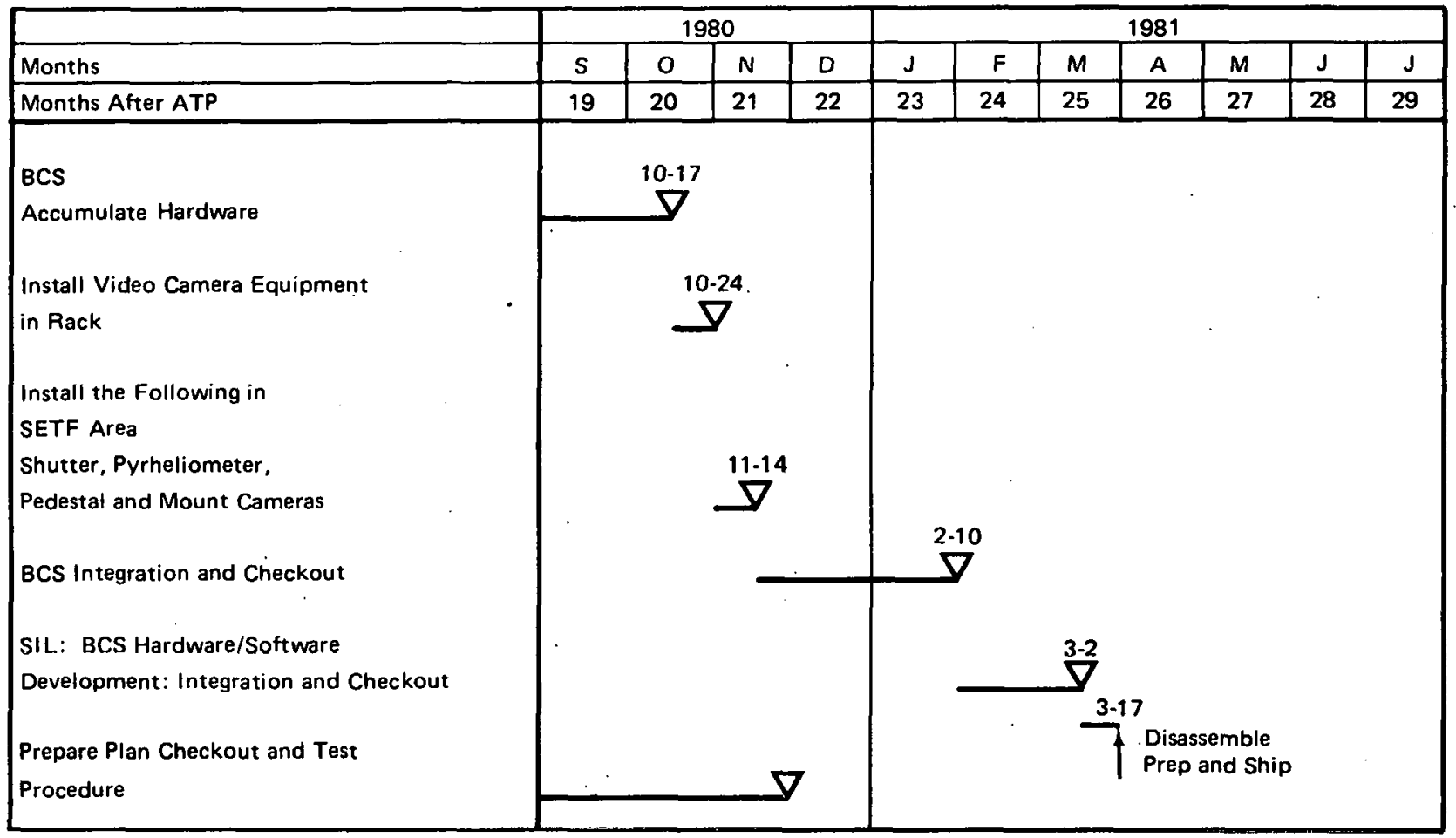

Figure 4-13. BCS System Overall Activities Schedule 


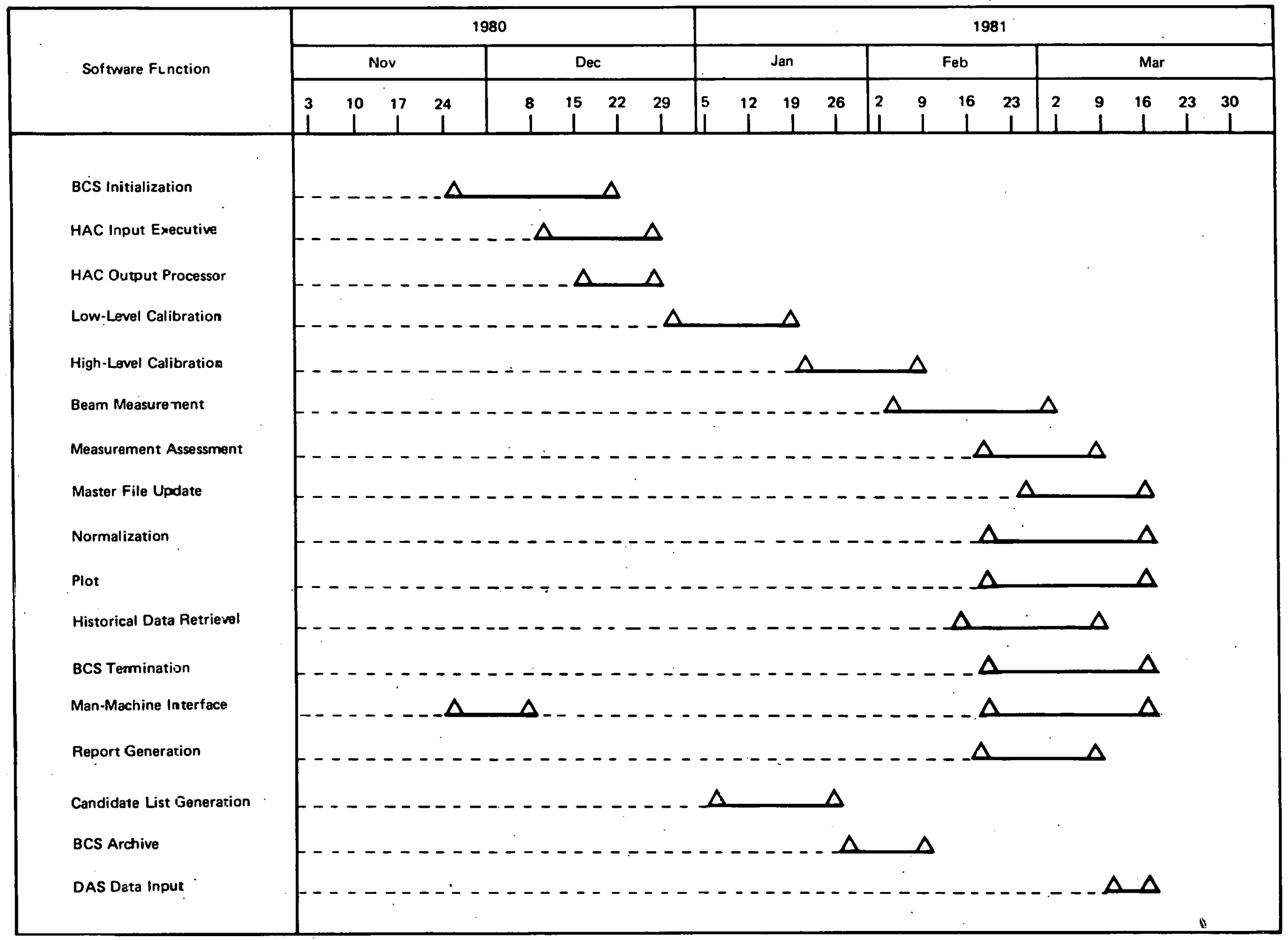

Figure 4-14. BCS Software Integration Test Scheaule 